\title{
Infrared Spectroscopic and Theoretical Studies of the 3d Transition Metal Oxyfluoride
}

\section{Molecules}

Rui Wei, ${ }^{\mathrm{a}, \mathrm{b}}$ Zongtang Fang, ${ }^{\mathrm{c}}$ Monica Vasiliu, ${ }^{\mathrm{c}}$ David A. Dixon, ${ }^{\mathrm{c}, *}$ Lester Andrews, ${ }^{\mathrm{d}}$ and Yu Gong, a,d,*

a Department of Radiochemistry, Shanghai Institute of Applied Physics, Chinese Academy of Sciences, Shanghai 201800, China

${ }^{\mathrm{b}}$ School of Nuclear Science and Technology, University of Chinese Academy of Sciences, Beijing 100049, China

c Department of Chemistry, The University of Alabama, Tuscaloosa, AL, 35487-0336, United States

d Department of Chemistry, University of Virginia, Charlottesville, VA, 22904-4319, United States

* Corresponding authors: dadixon@ua.ac (D.A.D.), gongyu@sinap.ac.cn (Y.G.). 


\section{Complete References}

Ref. 31: Gaussian 09, Frisch, M. J.; Trucks, G. W.; Schlegel, H. B.; Scuseria, G. E.; Robb, M. A.; Cheeseman, J. R.; Scalmani, G.; Barone, V.; Mennucci, B.; Petersson, G. A.; Nakatsuji, H.; Caricato, M.; Li, X.; Hratchian, H. P.; Izmaylov, A. F.; Bloino, J.; Zheng, G.; Sonnenberg, J. L.; Hada, M.; Ehara, M.; Toyota, K.; Fukuda, R.; Hasegawa, J.; Ishida, M.; Nakajima, T.; Honda, Y.; Kitao, O.; Nakai, H.; Vreven, T.; Montgomery, Jr., J. A.; Peralta, J. E.; Ogliaro, F.; Bearpark, M.; Heyd, J. J.; Brothers, E.; Kudin, K. N.; Staroverov, V. N.; Kobayashi, R.; Normand, J.; Raghavachari, K.; Rendell, A.; Burant, J. C.; Iyengar, S. S.; Tomasi, J.; Cossi, M.; Rega, N.; Millam, N. J.; Klene, M.; Knox, J. E.; Cross, J. B.; Bakken, V.; Adamo, C.; Jaramillo, J.; Gomperts, R.; Stratmann, R. E.; Yazyev, O.; Austin, A. J.; Cammi, R.; Pomelli, C.; Ochterski, J. W.; Martin, R. L.; Morokuma, K.; Zakrzewski, V. G.; Voth, G. A.; Salvador, P.; Dannenberg, J. J.; Dapprich, S.; Daniels, A. D.; Farkas, Ö.; Foresman, J. B.; Ortiz, J. V.; Cioslowski, J.; Fox, D. J. Gaussian, Inc., Wallingford CT, 2009.

Ref. 55: MOLPRO, version 2012.1, a package of ab initio programs, Werner H.-J.; Knowles, P. J.; Knizia, G.; Manby, F. R.; Schütz, M.; Celani, P.; Korona, T.; Lindh, R.; Mitrushenkov, A.; Rauhut, G.; Shamasundar K. R.; Adler, T. B.; Amos, R. D.; Bernhardsson, A.; Berning, A.; Cooper, D. L.; Deegan, M. J. O.; Dobbyn, A. J.; Eckert, F.; Goll, E.; Hampel, C.; Hesselmann, A.; Hetzer, G.; Hrenar, T.; Jansen, G.; Köppl, C.; Liu, Y.; Lloyd, A. W.; Mata, R. A.; May, A. J.; McNicholas, S. J.; Meyer, W.; Mura, M. E.; Nicklass, A.; O'Neill D. P.; Palmieri, P.; Peng, D.; Pflüger, K.; Pitzer, R.; Reiher, M.; Shiozaki, T.; Stoll, H.; Stone, A. J.; Tarroni, R.; Thorsteinsson, T.; Wang, M. See http://www.molpro.net. 


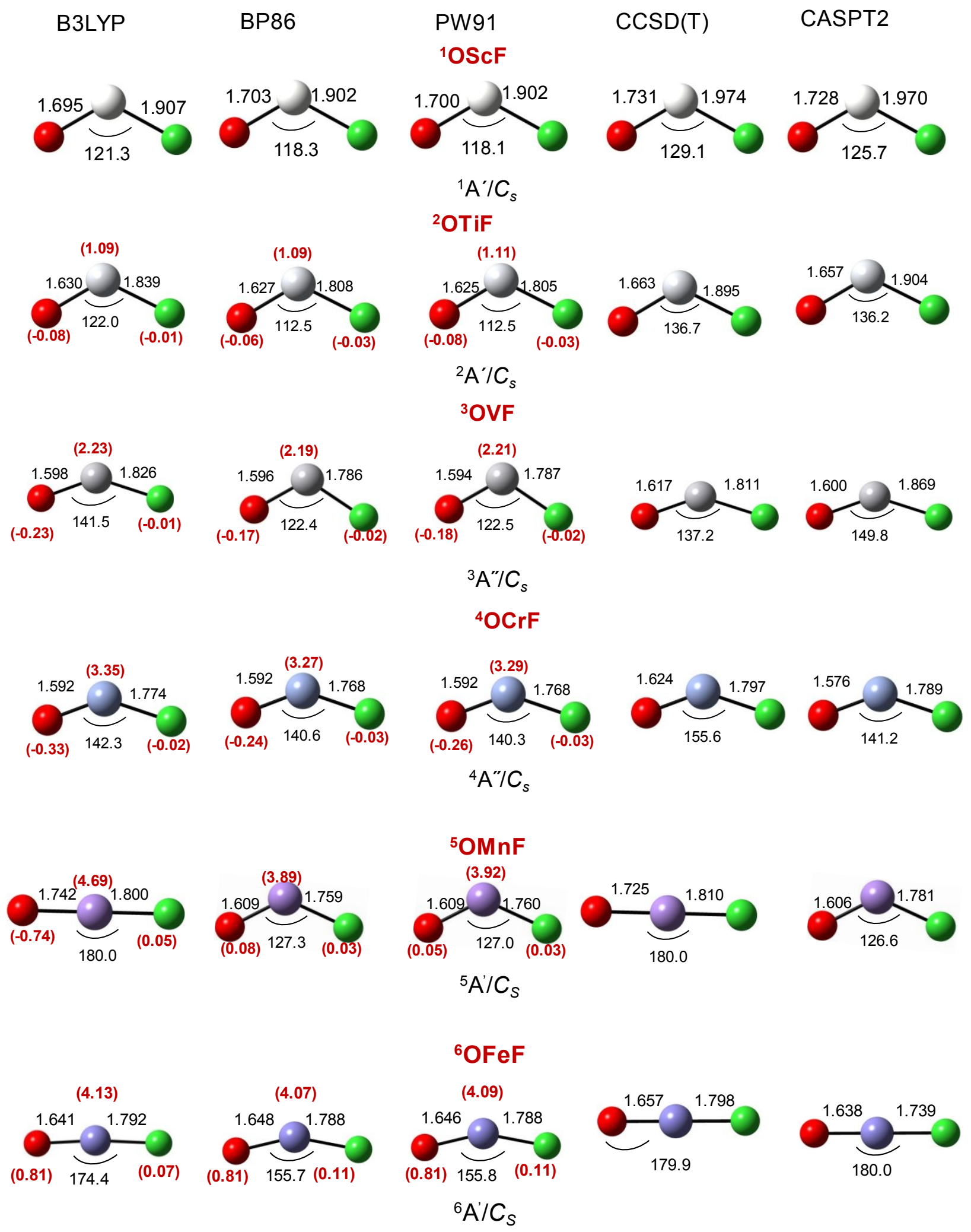




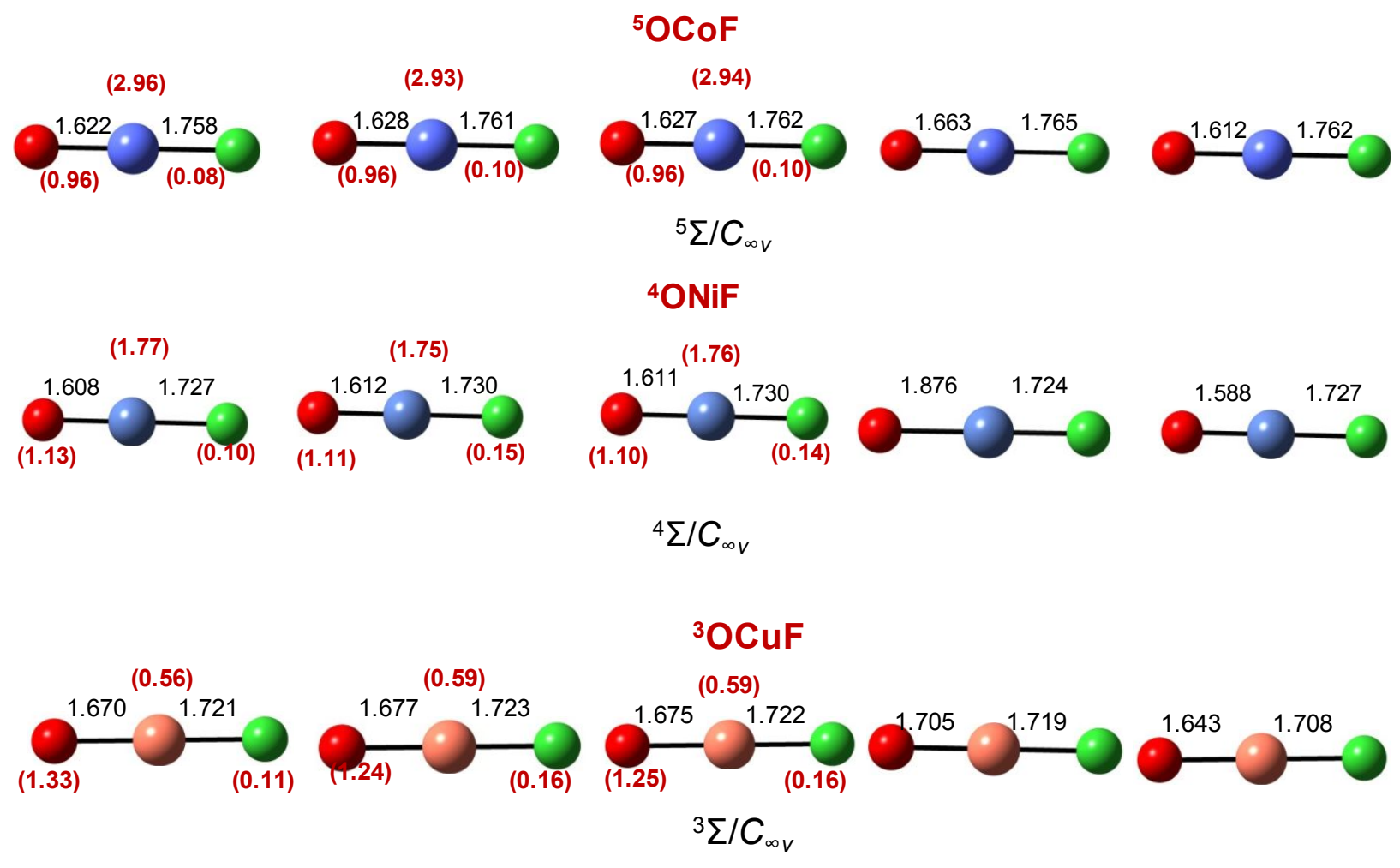

Figure S1. Molecular geometries and spins for OMF 

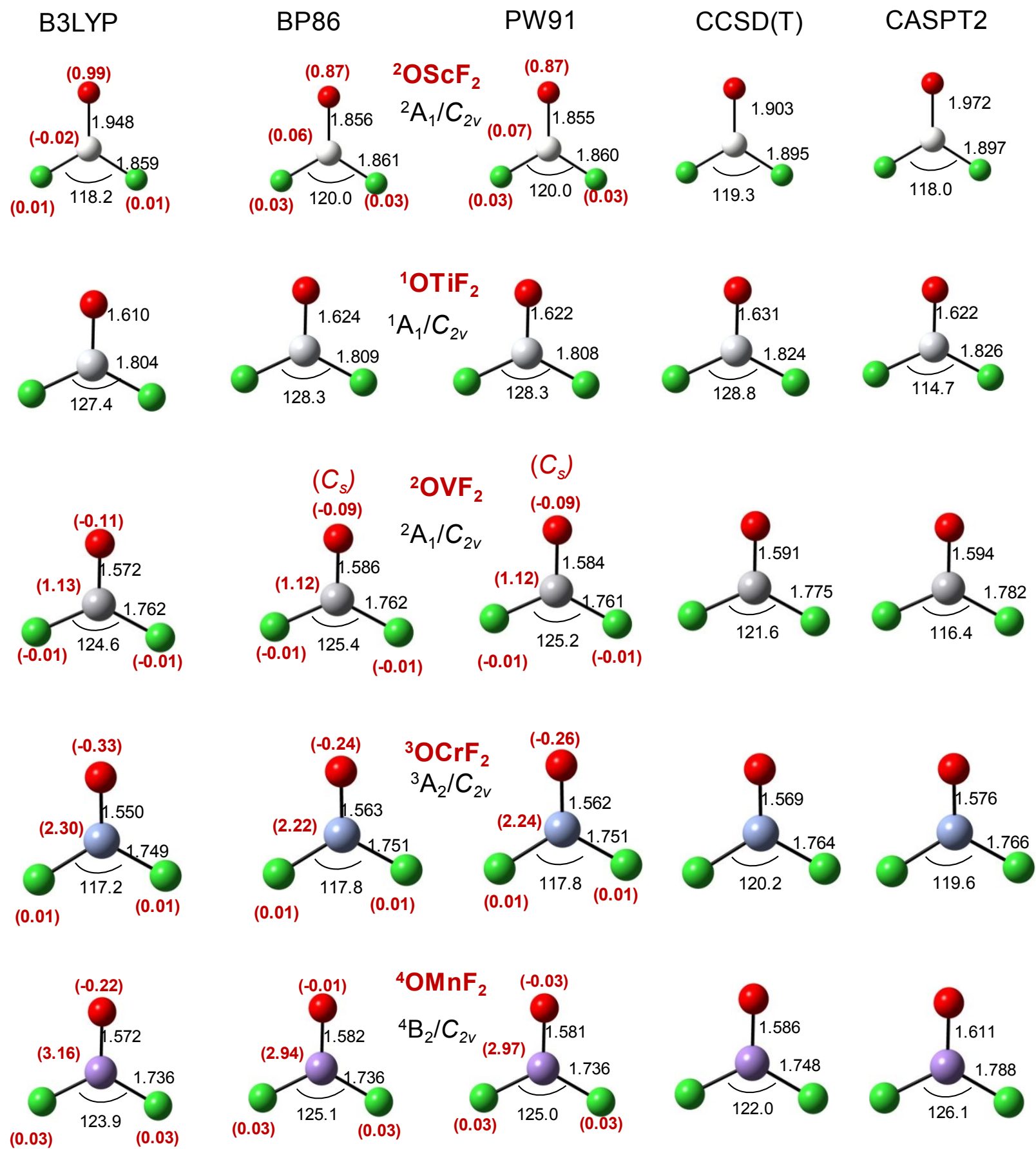


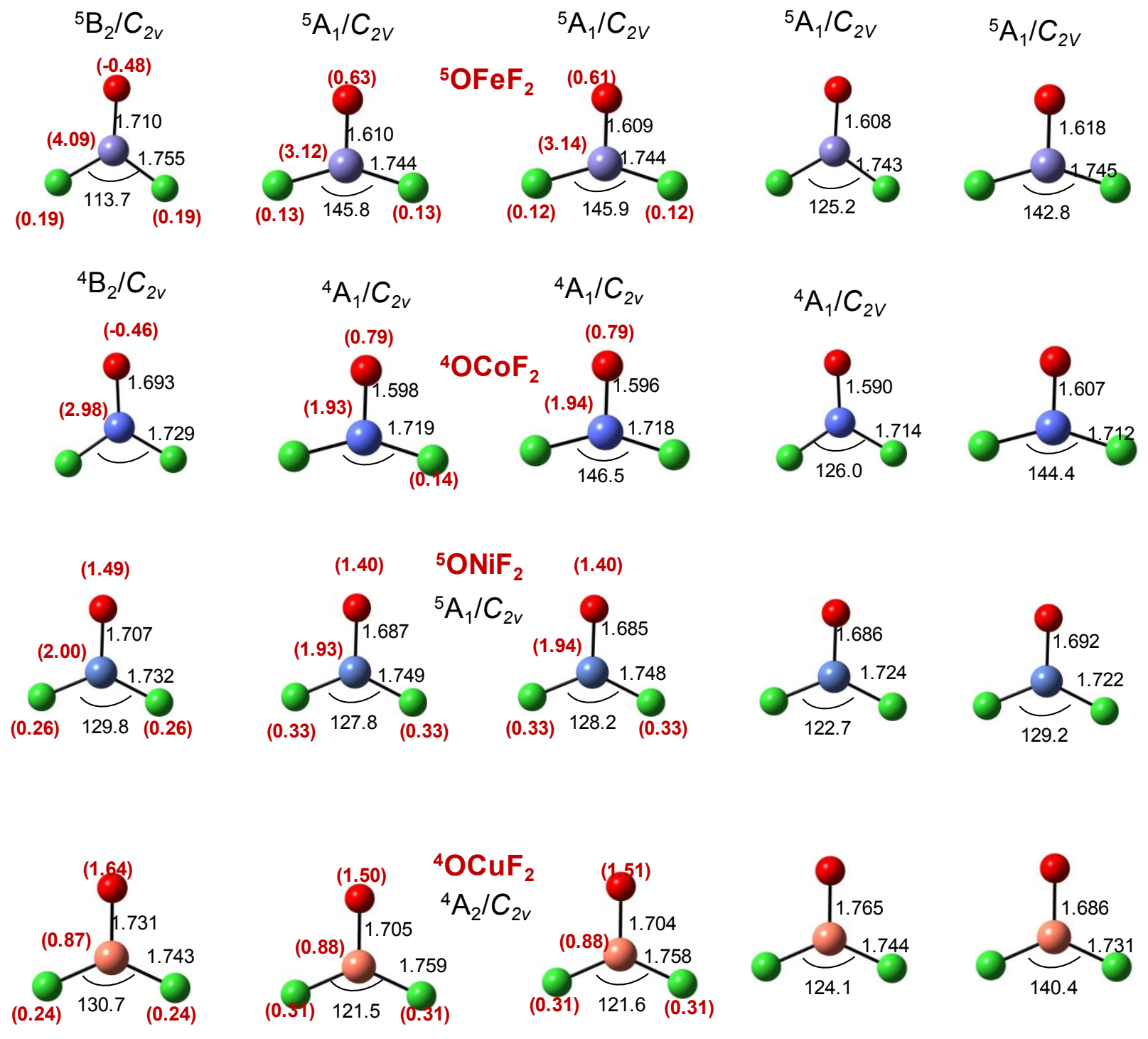

Figure S2. Molecular geometries and spins for $\mathrm{OMF}_{2}$ 
Table S1. Calculated Results for the OMF Molecules with Frequencies (v) in $\mathrm{cm}^{-1}$ and Infrared Intensities (I) in $\mathrm{km} / \mathrm{mol}$ at different level of theory.

\begin{tabular}{|c|c|c|c|c|c|c|c|c|c|}
\hline & ${ }^{1} \mathrm{OScF}$ & ${ }^{2} \mathrm{OTiF}$ & ${ }^{3} \mathrm{OVF}$ & ${ }^{4} \mathrm{OCrF}$ & ${ }^{5} \mathrm{OMnF}$ & ${ }^{6} \mathrm{OFeF}$ & ${ }^{5} \mathrm{OCoF}$ & ${ }^{4} \mathrm{ONiF}$ & ${ }^{3} \mathrm{OCuF}$ \\
\hline \multicolumn{10}{|c|}{ B3LYP } \\
\hline$v(\mathrm{M}-\mathrm{O})$ & 958.4 & 1019.1 & 1048.5 & 990.2 & 710.2 & 925.1 & 911.3 & 889.3 & $829.0 \mathrm{mix}$ \\
\hline $\mathrm{I}(\mathrm{M}-\mathrm{O})$ & 266 & 301 & 256 & 186 & 136 & 129 & 90 & 62 & 14 \\
\hline${ }^{16} \mathrm{O} /{ }^{18} \mathrm{O}$ & 1.0437 & 1.0436 & 1.0448 & 1.0418 & 1.0404 & 1.0398 & 1.0389 & 1.0355 & 1.0271 \\
\hline$v(\mathrm{M}-\mathrm{F})$ & 627.8 & 647.9 & 639.1 & 670.1 & 545.2 & 637.9 & 651.6 & 660.2 & $623.6 \mathrm{mix}$ \\
\hline $\mathrm{I}(\mathrm{M}-\mathrm{F})$ & 191 & 156 & 147 & 104 & 9 & 74 & 74 & 73 & 60 \\
\hline \multicolumn{10}{|c|}{ BP86 } \\
\hline$v(\mathrm{M}-\mathrm{O})$ & 929.7 & 1011.2 & 1028.6 & 999.6 & 948.9 & 899.2 & 905.5 & 906.3 & 815.7 \\
\hline $\mathrm{I}(\mathrm{M}-\mathrm{O})$ & 217 & 225 & 174 & 173 & 130 & 105 & 99 & 76 & 16 \\
\hline${ }^{16} \mathrm{O} /{ }^{18} \mathrm{O}$ & 1.04367 & 1.04377 & 1.04458 & 1.04244 & 1.04309 & 1.04110 & 1.03925 & 1.03922 & 1.02863 \\
\hline$v(\mathrm{M}-\mathrm{F})$ & 623.1 & 684.6 & 673.8 & 669.8 & 669.2 & 633.6 & 641.2 & 651.3 & 616.3 \\
\hline $\mathrm{I}(\mathrm{M}-\mathrm{F})$ & 178 & 182 & 140 & 91 & 86 & 67 & 57 & 51 & 31 \\
\hline \multicolumn{10}{|c|}{ CAS } \\
\hline$v(\mathrm{M}-\mathrm{O})$ & 938.3 & 993.4 & 1043.6 & 1091.8 & 998.2 & 983.6 & 764.7mix & 597.4 & - \\
\hline $\mathrm{I}(\mathrm{M}-\mathrm{O})$ & 301 & 310 & 215 & 445 & 182 & 303 & 140 & 3 & \\
\hline${ }^{16} \mathrm{O} /{ }^{18} \mathrm{O}$ & 1.0438 & 1.0442 & 1.0442 & 1.0435 & 1.0457 & 1.0424 & 1.0181 & 1.0422 & \\
\hline$v(\mathrm{M}-\mathrm{F})$ & 605.3 & 620.2 & 594.8 & 689.3 & 698.6 & 668.1 & $625.2 \mathrm{mix}$ & 756.1 & 626.8 \\
\hline $\mathrm{I}(\mathrm{M}-\mathrm{F})$ & 207 & 167 & 134 & 111 & 151 & 73 & 8 & 161 & 63 \\
\hline
\end{tabular}




\begin{tabular}{|l|l|l|l|l|l|l|l|l|l|l|}
\hline$v(\mathrm{M}-\mathrm{O})$ & 930.5 & 1001.6 & 1000.3 & 943.0 & 801.6 & 883.3 & $599.1 \mathrm{mix}$ & $636.9 \mathrm{mix}$ & $838.6 \mathrm{mix}$ \\
\hline${ }^{16} \mathrm{O} /{ }^{18} \mathrm{O}$ & 1.0432 & 1.0444 & 1.0418 & 1.0376 & 1.0136 & 1.0341 & & & 1.0237 \\
\hline$v(\mathrm{M}-\mathrm{F})$ & 633.4 & 731.8 & 700.3 & 674.3 & 638.5 & 653.7 & $761.2 \mathrm{mix}$ & $785.5 \mathrm{mix}$ & $677.7 \mathrm{mix}$ \\
\hline \multicolumn{2}{|l|}{ CASPT2/aVTZ-DK(O)/VDZ-DK(M) } \\
\hline$v(\mathrm{M}-\mathrm{O})$
\end{tabular}


Table S2. Calculated Results for the $\mathrm{OMF}_{2}$ Molecules with Frequencies (v) in $\mathrm{cm}^{-1}$ and Infrared Intensities (I) in $\mathrm{km} / \mathrm{mol}$ at different levels of theory.

\begin{tabular}{|c|c|c|c|c|c|c|c|c|c|}
\hline & ${ }^{2} \mathrm{OScF}_{2}$ & ${ }^{1} \mathrm{OTiF}_{2}$ & ${ }^{2} \mathrm{OVF}_{2}$ & ${ }^{3} \mathrm{OCrF}_{2}$ & ${ }^{4} \mathrm{OMnF}_{2}$ & ${ }^{5} \mathrm{OFeF}_{2}$ & ${ }^{4} \mathrm{OCoF}_{2}$ & ${ }^{5} \mathrm{ONiF}_{2}$ & ${ }^{4} \mathrm{OCuF}_{2}$ \\
\hline \multicolumn{10}{|c|}{ B3LYP } \\
\hline$v(\mathrm{M}-\mathrm{O})$ & 603.3 & 1079.1 & 1106.2 & 1085.4 & 984.9 & 741.9 & 723.3 & $677.2 \mathrm{mix}$ & 568.6 \\
\hline $\mathrm{I}(\mathrm{M}-\mathrm{O})$ & 2 & 256 & 215 & 17 & 94 & 88 & 91 & 20 & 30 \\
\hline${ }^{16} \mathrm{O} /{ }^{18} \mathrm{O}$ & 1.0292 & 1.0429 & 1.0440 & 1.0438 & 1.0424 & 1.0108 & 1.0096 & 1.0264 & 1.0331 \\
\hline$v(\mathrm{M}-\mathrm{F})$ & 717.2 & 777.8 & 782.2 & 749.7 & 767.2 & 722.1 & 716.2 & 725.1 & 671.8 \\
\hline $\mathrm{I}(\mathrm{M}-\mathrm{F})$ & 309 & 276 & 227 & 219 & 181 & 151 & 139 & 131 & 92 \\
\hline$v(\mathrm{M}-\mathrm{F})$ & 690.7 & 662.9 & 667.4 & 676.3 & 656.2 & 565.5 & 589.8 mix & 616.4 & 628.6 \\
\hline I(M-F) & 238 & 72 & 69 & 72 & 57 & 8 & 1 & 36 & 9 \\
\hline \multicolumn{10}{|c|}{ BP86 } \\
\hline$v(\mathrm{M}-\mathrm{O})$ & 602.9 & 1035.5 & 1064.7 & 1065.4 & 1008.2 & 930.8 & 924.6 & 740.1 & 683.3 \\
\hline $\mathrm{I}(\mathrm{M}-\mathrm{O})$ & 1 & 209 & 160 & 160 & 107 & 38 & 22 & 2 & 2 \\
\hline${ }^{16} \mathrm{O} /{ }^{18} \mathrm{O}$ & 1.02796 & 1.04301 & 1.04362 & 1.04338 & 1.04379 & 1.04561 & 1.04652 & 1.04460 & 1.04035 \\
\hline$v(\mathrm{M}-\mathrm{F})$ & 693.4 & 756.2 & 777.7 & 737.1 & 759.3 & 747.7 & 753.0 & 677.4 & 626.4 \\
\hline $\mathrm{I}(\mathrm{M}-\mathrm{F})$ & 195 & 244 & 196 & 196 & 155 & 176 & 138 & 73 & 59 \\
\hline$v(\mathrm{M}-\mathrm{F})$ & 668.7 & 652.6 & 660.3 & 662.9 & 651.4 & 622.3 & 625.0 & 592.0 & 565.0 \\
\hline I(M-F) & 164 & 67 & 53 & 53 & 42 & 21 & 17 & 22 & 14 \\
\hline \multicolumn{10}{|c|}{ CAS } \\
\hline$v(\mathrm{M}-\mathrm{O})$ & 761.5 & 1057.2 & 1098.6 & 1076.0 & 862.3 & 890.9 & 886.2 & 199.8 & 160.6 \\
\hline $\mathrm{I}(\mathrm{M}-\mathrm{O})$ & 283.2 & 142 & 126.7 & 92 & 44 & 106 & 5 & 35 & 3 \\
\hline
\end{tabular}




\begin{tabular}{|l|l|l|l|l|l|l|l|l|l|}
\hline${ }^{16} \mathrm{O} /{ }^{18} \mathrm{O}$ & 1.0339 & 1.0427 & 1.0440 & 1.0439 & 1.0398 & 1.0434 & 1.0459 & & \\
\hline$v(\mathrm{M}-\mathrm{F})$ & 681.8 & 794.3 & 780.0 & 767.1 & 775.2 & 806.8 & 796.2 & 768.4 & 779.8 \\
\hline $\mathrm{I}(\mathrm{M}-\mathrm{F})$ & 89 & 91 & 86 & 85 & 80 & 76 & 221 & 73 & 71 \\
\hline$v(\mathrm{M}-\mathrm{F})$ & 620.1 & 680.7 & 674.1 & 682.4 & 650.7 & 692.8 & 666.2 & 621.6 & 648.3 \\
\hline $\mathrm{I}(\mathrm{M}-\mathrm{F})$ & 42 & 98 & 105 & 107 & 73 & 46 & 79 & 2 & 1 \\
\hline
\end{tabular}


Table S3. CI vectors of the lowest energy state ground states $\mathrm{OMF}$ and $\mathrm{OMF}_{2}$ molecules.

The order for $\mathrm{C}_{s}$ is $\mathrm{a}^{\prime}, \mathrm{a}^{\prime \prime}$

The order for $\mathrm{C}_{2 v}$ is $\mathrm{a}_{1}, \mathrm{~b}_{1}, \mathrm{~b}_{2}, \mathrm{a}_{2}$. For a linear molecule this corresponds to $\mathrm{a}_{1}$ is sigma; $\mathrm{b}_{1}, \mathrm{~b}_{2}$ are pi; $\mathrm{a}_{1}, \mathrm{a}_{2}$ are delta

The " 2 " means double occupied orbitals, "a" means a single alpha electron, "b" means a single beta electron and " 0 " means unoccupied.

${ }^{1}$ OScF CI vector

$\begin{array}{lr}222200002200 & 0.9532870 \\ 222200002020 & -0.0910449 \\ 222020002200 & -0.0882439 \\ 220202002200 & -0.0726318 \\ \text { 222ba000 2ab0 } & -0.0619600 \\ \text { 222ab000 2ba0 } & -0.0619600 \\ \text { 22220000 0202 } & -0.0604692 \\ \text { 22abab00 2200 } & 0.0571291 \\ \text { 22baba00 2200 } & 0.0571291 \\ \text { 22a20b00 2ba0 } & -0.0561198 \\ \text { 22b20a00 2ab0 } & -0.0561198\end{array}$

${ }^{2}$ OTiF CI vector

$2222 \mathrm{a} 0002200$

0.9521475

2220a200 2200

$-0.1040855$

2202a020 2200

22abaab0 2200

$-0.0854829$

22 baaba 02200

0.0704425

$2222 \mathrm{a} 0000202$

0.0702739

22 bbaaa 2200

$-0.0651268$

$2022 \mathrm{a} 0022200$

222aab00 $2 \mathrm{ba} 0$

$-0.0592968$

$-0.0584121$

0.0584093

$2222 \mathrm{a} 0002020$

$-0.0567264$

22aaabb0 2200

$-0.0557208$

$222 \mathrm{baa} 002 \mathrm{ab} 0$

0.0552500

$222 \mathrm{baa} 002 \mathrm{ba} 0$

$-0.0518067$

${ }^{4} \mathrm{OCrF}$ CI vector

$\begin{array}{cc}222 \mathrm{aa} 0022 \mathrm{a} 0 & 0.9040147 \\ 222 \mathrm{aa} 002 \mathrm{baa} & 0.1640481 \\ 222 \mathrm{aa} 0020 \mathrm{a} 2 & -0.1232983 \\ 2220 \mathrm{aa} 202 \mathrm{a} 0 & -0.1206454 \\ 222 \mathrm{aaab0} 2 \mathrm{baa} & 0.1024612 \\ 222 \mathrm{baaa} 02 \mathrm{baa} & -0.0916003\end{array}$




$\begin{array}{lr}222 \mathrm{baaa} 02 \mathrm{aab} & 0.0863232 \\ 222 \mathrm{baaa} 022 \mathrm{a} 0 & 0.0838801 \\ 222 \mathrm{ba} 002 \mathrm{aaa} & -0.0619934 \\ 2222 \mathrm{aa} 002 \mathrm{aba} & -0.0606917 \\ 22 \mathrm{a} 2 \mathrm{a} 2002 \mathrm{baa} & 0.0597406 \\ 2202 \mathrm{aa} 0222 \mathrm{a} 0 & -0.0577165 \\ 22 \mathrm{abaaab} 22 \mathrm{a} 0 & 0.0564238 \\ 22 \mathrm{baaaba} 22 \mathrm{a} 0 & 0.0563957 \\ \text { 22a2aa0b 2baa } & 0.0562518 \\ \text { 22b2aa0a 22a0 } & 0.0521177 \\ \text { 222aaab0 2aab } & -0.0520672 \\ \text { 22aba2a0 22a0 } & -0.0505515\end{array}$

${ }^{5}$ OMnF CI vector (degenerate) state symmetry 1

$\begin{array}{lr}\text { 2222aa00 22aa } & -0.6933446 \\ \text { 222baaa 22aa } & 0.5271154 \\ \text { 2220aa20 22aa } & 0.2672992 \\ \text { 222baaa0 2aa2 } & -0.2050137 \\ \text { 222aaba0 22aa } & -0.1341268 \\ \text { 222aaaa0 22ba } & -0.1341261 \\ \text { 222aaaa0 22ab } & -0.1203841 \\ \text { 222abaa0 22aa } & -0.1201353 \\ \text { 2222aa00 2aa2 } & 0.1091432 \\ \text { 2220aa20 2aa2 } & -0.0859452 \\ \text { 22abaaab 22aa } & 0.0649682 \\ \text { 22b2aa0a 22aa } & -0.0551967 \\ \text { 2202aa02 22aa } & 0.0519977 \\ \text { 222aaaa0 2ba2 } & 0.0500945\end{array}$

state symmetry 2

$\begin{array}{lc}\text { 2222aaa0 22a0 } & 0.6933303 \\ \text { 2222aaa0 2baa } & 0.5271287 \\ \text { 2222aaa0 20a2 } & -0.2673042 \\ \text { 222aaa20 2baa } & 0.2050139 \\ \text { 2222aaa0 2aba } & -0.1341291 \\ \text { 2222aba0 2aaa } & -0.1341284 \\ \text { 2222aab0 2aaa } & -0.1203873 \\ \text { 2222baa0 2aaa } & -0.1201414 \\ \text { 222aaa20 22a0 } & 0.1091458 \\ \text { 222aaa20 20a2 } & -0.0859454 \\ \text { 22a2aaab 2baa } & -0.0649674 \\ \text { 22b2aaaa 22a0 } & -0.0551946 \\ \text { 2202aaa2 22a0 } & -0.0519967\end{array}$


222baa20 2aaa $\quad-0.0500951$

${ }^{6} \mathrm{OFeF}$ CI vector

$\begin{array}{lr}22 \mathrm{aa} 022 \mathrm{a} 22 \mathrm{a} a & 0.7689196 \\ 22 \mathrm{aa} 02 \mathrm{a} 2 \text { 22a a } & -0.3783339 \\ 22 \mathrm{aa} 022 \mathrm{a} 2 \mathrm{a} 2 \mathrm{a} & -0.3783339 \\ 2 \mathrm{a} 2 \mathrm{a} 022 \mathrm{a} 22 \mathrm{a} a & 0.2282168 \\ 2 \mathrm{baaa} 22 \mathrm{a} 22 \mathrm{a} a & 0.1398047 \\ 22 \mathrm{aa} 02 \mathrm{a} 22 \mathrm{a} 2 \mathrm{a} & -0.1195890 \\ 2 \mathrm{a} 2 \mathrm{a} 02 \mathrm{a} 222 \mathrm{a} a & 0.0728622 \\ 2 \mathrm{a} 2 \mathrm{a} 022 \mathrm{a} 2 \mathrm{a} 2 \mathrm{a} & 0.0728622\end{array}$

${ }^{5} \mathrm{OCoF}$ CI vector

$\begin{array}{lr}222 \mathrm{a} 022 \mathrm{a} 22 \mathrm{a} a & -0.9061121 \\ 222 \mathrm{a} 02 \mathrm{a} 2 \text { 2a2 a } & 0.2925426 \\ 2 \mathrm{a} 22022 \mathrm{a} 2 \mathrm{a} 2 \mathrm{a} & -0.1192137 \\ 2 \mathrm{a} 2202 \mathrm{a} 222 \mathrm{a} a & -0.1192136 \\ 2 \mathrm{a} 22022 \mathrm{a} 22 \mathrm{a} a & -0.0915789 \\ 2 \mathrm{a} 2202 \mathrm{a} 22 \mathrm{a} 2 \mathrm{a} & -0.0849015 \\ 22 \mathrm{aa} 02 \mathrm{aa} 222 \mathrm{a} & 0.0764061 \\ 22 \mathrm{aa} 02222 \mathrm{aa} 2 & 0.0764060 \\ 22 \mathrm{a} 202 \mathrm{a} 22 \mathrm{a} a & -0.0664363 \\ 22 \mathrm{a} 2022 \mathrm{a} 2 \mathrm{a} 2 \mathrm{a} & 0.0664362 \\ 2 \mathrm{~b} 2 \mathrm{aa} 22 \mathrm{a} 2 \mathrm{a} 2 \mathrm{a} & 0.0587087 \\ 2 \mathrm{~b} 2 \mathrm{aa} 2 \mathrm{a} 222 \mathrm{a} a & 0.0587087 \\ 2 \mathrm{a} 2 \mathrm{ab} 22 \mathrm{a} 2 \mathrm{a} 2 \mathrm{a} & -0.0539200 \\ 2 \mathrm{a} 2 \mathrm{ab} 2 \mathrm{a} 222 \mathrm{a} a & -0.0539199 \\ 202 \mathrm{a} 222 \mathrm{a} 22 \mathrm{a} a & 0.0522813\end{array}$

CI vector for state symmetry 4

\begin{tabular}{|c|c|}
\hline $22 \mathrm{aa} 022 \mathrm{a} 22 \mathrm{a}$ & \\
\hline $22 \mathrm{aa} 02 \mathrm{a} 22 \mathrm{a} 22$ & -0.2925428 \\
\hline $2 \mathrm{aa} 2022 \mathrm{a} 2 \mathrm{a} 2$ & -0.1 \\
\hline $2 \mathrm{aa} 202 \mathrm{a} 222 \mathrm{a} 2$ & -0.1192 \\
\hline $2 \mathrm{aa} 2022 \mathrm{a} 22 \mathrm{a} 2$ & -0.09157 \\
\hline $2 \mathrm{aa} 202 \mathrm{a} 22 \mathrm{a} 22$ & -0.084901 \\
\hline $22 \mathrm{aa} a$ & 0.07640 \\
\hline $2 \mathrm{a} 0$ & 0.076406 \\
\hline $22 \mathrm{a} 20$ & -0.0664362 \\
\hline $22 \mathrm{a} 20$ & 0.066436 \\
\hline taa & 0.058 \\
\hline 2 baaa & 0.05870 \\
\hline $2 \mathrm{aaab}$ & -0.0539 \\
\hline
\end{tabular}


$2 \mathrm{aaab} 22 \mathrm{a} 2 \mathrm{a} 2 \mathrm{C}-0.0539199$

20aa2 22a 22a $2 \quad-0.0522813$

${ }^{4} \mathrm{ONiF}$ CI vector

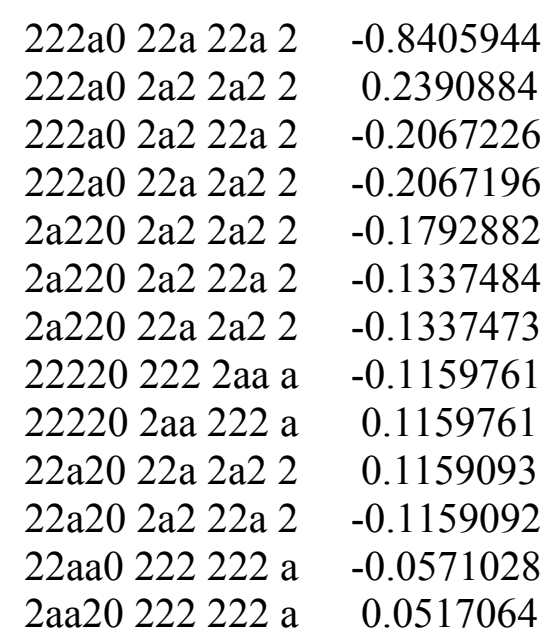

${ }^{3} \mathrm{OCuF}$ CI vector

$2222022 \mathrm{a} 22 \mathrm{a} 2 \quad 0.7468510$

$2222022 \mathrm{a} 2 \mathrm{a} 22 \quad 0.4086401$

$222202 \mathrm{a} 222 \mathrm{a} 2 \quad-0.4081853$

$222202 \mathrm{a} 22 \mathrm{a} 22 \quad 0.3043845$ 
${ }^{2} \mathrm{OScF}_{2} \mathrm{CI}$ vector

$\begin{array}{cc}200020 \mathrm{a} 00 & 0.9806558 \\ 200002 \mathrm{a} 00 & -0.0764470 \\ 002020 \mathrm{a} 00 & -0.0628611 \\ 2000 \mathrm{ab} \text { a0 } 0 & 0.0567479 \\ 2000 \mathrm{ab} 0 \mathrm{a} 0 & -0.0541682\end{array}$

${ }^{1} \mathrm{OTiF}_{2} \mathrm{CI}$ vector

$\begin{array}{ll}200020200 & 0.9559795 \\ 200002200 & -0.1101258 \\ 020020200 & -0.1003784 \\ 200020020 & -0.0835875 \\ \text { ba00 ab 20 0 } & -0.0744777 \\ \text { ab00 ba } 200 & -0.0744777 \\ 2000 \text { ba ab } 0 & -0.0648369 \\ 2000 \text { ab ba } 0 & -0.0648369 \\ \text { ab00 20 ba } 0 & 0.0627198 \\ \text { ba00 20 ab } 0 & 0.0627198 \\ \text { ba00 ba } 200 & 0.0591103 \\ \text { ab00 ab 20 } 0 & 0.0591103\end{array}$

${ }^{2} \mathrm{OVF}_{2} \mathrm{CI}$ vector

$\begin{array}{lr}2 \mathrm{a} 0020200 & 0.9257420 \\ 2 \mathrm{a} 0002200 & -0.1351653 \\ 20 \mathrm{a} 020200 & 0.0934923 \\ 0 \mathrm{a} 2020200 & -0.0933269 \\ 2 \mathrm{a} 0020020 & -0.0932925 \\ 2 \mathrm{a} 00 \mathrm{ab} \text { ba } 0 & -0.0824514 \\ 2 \mathrm{a} 00 \text { ba ab } 0 & -0.0809447 \\ \mathrm{baa} 0 \mathrm{ab} 200 & -0.0808083 \\ \mathrm{a} a b 0 \text { ba } 200 & -0.0798734 \\ \mathrm{baa} 0 \text { ba } 200 & 0.0713319 \\ \mathrm{aab} 020 \text { ba } 0 & 0.0711453 \\ \mathrm{baa} 020 \text { ab } 0 & 0.0698806 \\ 2 \mathrm{a} 00 \text { ba ba } 0 & 0.0688367 \\ \mathrm{baa} 020 \text { ba } 0 & -0.0644740 \\ \text { 2b00 aa } 200 & 0.0586756 \\ \mathrm{a} 0 \mathrm{ab} 0 \mathrm{ab} 200 & 0.0583417 \\ 2 \mathrm{a} 00 \mathrm{ab} \text { ab } 0 & 0.0552516 \\ \mathrm{aab} 020 \text { ab } 0 & -0.0546718 \\ 2 \mathrm{a} 0020 \text { ba } 0 & 0.0504617\end{array}$


${ }^{3} \mathrm{OCrF}_{2} \mathrm{CI}$ vector

$\begin{array}{lc}2 \mathrm{a} 002020 \mathrm{a} & 0.9089564 \\ 2 \mathrm{a} 000220 \mathrm{a} & -0.1417534 \\ 2 \mathrm{a} 002002 \mathrm{a} & -0.0987103 \\ 0 \mathrm{a} 202020 \mathrm{a} & -0.0868196 \\ 2 \mathrm{a} 00 \text { ba ab a } & 0.0843244 \\ \mathrm{aab} 0 \text { ba } 20 \mathrm{a} & 0.0841701 \\ 2 \mathrm{a} 00 \text { ba } 20 \mathrm{a} & -0.0792404 \\ 2 \mathrm{a} 00 \mathrm{ab} \text { ba a } & 0.0766004 \\ \mathrm{baa} 0 \mathrm{ab} 20 \mathrm{a} & 0.0762239 \\ \mathrm{baa} 0 \text { ba } 20 \mathrm{a} & -0.0752735 \\ 2 \mathrm{a} 00 \text { aa } 20 \mathrm{~b} & 0.0722281 \\ 2 \mathrm{a} 00 \text { ba ba a } & -0.0704170 \\ \mathrm{baa} 020 \mathrm{ab} \mathrm{a} & 0.0679924 \\ 20 \mathrm{a} 02020 \mathrm{a} & -0.0675412 \\ \mathrm{aab} 020 \text { ba a } & 0.0664367 \\ \mathrm{baa} 020 \text { ba a } & -0.0654704 \\ 2 \mathrm{~b} 00 \text { aa } 20 \mathrm{a} & 0.0632851 \\ 2 \mathrm{a} 0020 \text { ab a } & -0.0622485 \\ 2 \mathrm{a} 00 \text { ab } 20 \mathrm{a} & -0.0562728 \\ \mathrm{aaa} 02020 \mathrm{~b} & 0.0527248\end{array}$

${ }^{4} \mathrm{OMnF}_{2} \mathrm{CI}$ vector

\begin{tabular}{|c|c|}
\hline & \\
\hline $\mathrm{a} 002 \mathrm{a} b a \mathrm{a}$ & \\
\hline-100 & -0.1 \\
\hline $0 \mathrm{a}$ & 831 \\
\hline & -0.15 \\
\hline & -0 . \\
\hline & \\
\hline & \\
\hline 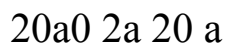 & 0.1 \\
\hline & 0 . \\
\hline & \\
\hline & 0.0 \\
\hline 100 & 0.0 \\
\hline & -0.0 \\
\hline & \\
\hline & 0.061870 \\
\hline & 0.06076 \\
\hline & -0.0593 \\
\hline
\end{tabular}




$$
\begin{array}{lr}
\text { aba0 2a 20 a } & 0.0574290 \\
\text { aab0 2a ab a } & -0.0559575 \\
\text { aab0 a2 20 a } & 0.0546857
\end{array}
$$

${ }^{5} \mathrm{OFeF}_{2} \mathrm{CI}$ vector

$$
\begin{aligned}
& \text { 2aa0 2a } 20 \text { a } \quad 0.7415234 \\
& 2 \mathrm{aa} 02 \mathrm{a} \text { ba a } \quad-0.4785734 \\
& 2 \mathrm{aa} 02 \mathrm{a} 02 \mathrm{a} \quad-0.2543081 \\
& 2 \mathrm{aa} 0 \text { a2 ba a } \quad-0.2099217 \\
& 2 \mathrm{ba} 02 \mathrm{a} \text { aa a } \quad 0.1379974 \\
& 2 \mathrm{aa} 02 \mathrm{a} \text { aa b } \quad 0.1344830 \\
& \text { aa20 2a ba a } \quad-0.1289091 \\
& 2 \mathrm{aa} 02 \mathrm{~b} \text { aa a } \quad 0.0934235 \\
& 2 \mathrm{ab0} 2 \mathrm{a} \text { aa a } \quad 0.0819324 \\
& 2 \mathrm{aa} 0 \text { a2 } 20 \text { a } \quad 0.0666242 \\
& 2 \mathrm{aa} 0 \mathrm{a} 2 \mathrm{ab} \text { a } \quad 0.0662767 \\
& 2 \mathrm{aa} 0 \text { a2 } 02 \text { a } \quad-0.0662040 \\
& 2 \mathrm{aa} 0 \mathrm{~b} 2 \text { aa a } \quad 0.0608754 \\
& \text { aa20 a2 } 20 \text { a } \quad-0.0529652 \\
& \text { aa20 } 2 \mathrm{a} \text { ab a } \quad 0.0528170
\end{aligned}
$$

${ }^{4} \mathrm{OCoF}_{2}$ CI vector

$\begin{array}{lr}22002 \mathrm{a} 2 \mathrm{a} a & 0.8863040 \\ \mathrm{~b} 2 \mathrm{a} 0 \text { 2a } 2 \mathrm{a} a & 0.1701559 \\ 2200 \mathrm{a} 2 \mathrm{a} 2 \mathrm{a} & -0.1597451 \\ 02202 \mathrm{a} 2 \mathrm{a} a & -0.1524874 \\ \mathrm{~b} 2 \mathrm{a} 0 \mathrm{a} 22 \mathrm{a} a & 0.1489484 \\ \mathrm{~b} 2 \mathrm{a} 0 \text { 2a } 2 \mathrm{a} & 0.1440356 \\ \mathrm{a} 2 \mathrm{~b} 02 \mathrm{a} \text { a2 a } & -0.1086709 \\ \mathrm{a} 2 \mathrm{~b} 0 \mathrm{a} 22 \mathrm{a} a & -0.1066330 \\ \mathrm{a} 2 \mathrm{a} 02 \mathrm{a} 2 \mathrm{a} \mathrm{b} & -0.0925987 \\ 2 \mathrm{ba} 02 \mathrm{a} 2 \mathrm{a} a & -0.0779263 \\ 0220 \mathrm{a} 2 \mathrm{a} 2 \mathrm{a} & 0.0632037 \\ 22002 \mathrm{a} 2 \mathrm{a} & -0.0627511 \\ 2 \mathrm{a} 00 \mathrm{aa} 22 \mathrm{2} & 0.0623440 \\ 20202 \mathrm{a} 2 \mathrm{a} a & -0.0586616 \\ 2 \mathrm{ba} 02 \mathrm{a} 2 \mathrm{a} & -0.0509543\end{array}$

${ }^{5} \mathrm{ONiF}_{2} \mathrm{CI}$ vector

$\begin{array}{lr}2 \mathrm{aa} 0 \text { 2a 2a } 2 & 0.9042954 \\ \text { 2aa0 a2 a2 2 } & -0.2510574 \\ \text { 2aa0 a2 2a 2 } & -0.1805659\end{array}$ 


$$
\begin{array}{lr}
\mathrm{aa} 20 \text { 2a a2 } 2 & -0.1417339 \\
22 \mathrm{a} 0 \text { 22 aa a } & 0.1363563 \\
\mathrm{aa} 20 \text { a2 } 2 \mathrm{a} 2 & -0.1156429 \\
\mathrm{a} 2 \mathrm{a} 0 \mathrm{a} 2 \text { 2a } 2 & -0.0859658 \\
2 \mathrm{a} 20 \text { a } 22 \mathrm{a} & -0.0820209
\end{array}
$$

\begin{tabular}{|c|c|}
\hline $22 \mathrm{a} 02 \mathrm{a} 2 \mathrm{a} 2$ & 0.8760929 \\
\hline $\mathrm{a} 2202 \mathrm{a} 2 \mathrm{a} 2$ & -0.2661782 \\
\hline $2 \mathrm{a} 202 \mathrm{a} a 22$ & -0.2327828 \\
\hline $22 \mathrm{a} 02 \mathrm{a} a 22$ & -0.1791623 \\
\hline $2 \mathrm{a} 20 \mathrm{a} 22 \mathrm{a} 2$ & -0.1480527 \\
\hline $2 \mathrm{a} 202 \mathrm{a} 2 \mathrm{a} 2$ & -0.1244714 \\
\hline $22 \mathrm{a} 0 \mathrm{a} 2 \mathrm{a} 2$ & -0.1193604 \\
\hline a220 2a a2 2 & -0.0773848 \\
\hline $\mathrm{a} 220 \mathrm{a} 2 \mathrm{2a} 2$ & -0.0705978 \\
\hline רת רת & -0.0573631 \\
\hline
\end{tabular}

${ }^{4} \mathrm{OCuF}_{2}$ CI vector 
Table S4. CAS orbital types and occupancies for $\mathrm{OMF}$ and $\mathrm{OMF}_{2}$ ground states.

${ }^{1} \mathrm{OScF}$

Orbital Occupation Energy Coefficients

$\begin{array}{llllll}1.1 & 2.00000 & -166.81273 & 1 & 1 \mathrm{~s} & 0.97310\end{array}$

$\begin{array}{lllll}2.1 & 2.00000 & -26.26262 & 31 \mathrm{~s} & 1.00060\end{array}$

$\begin{array}{lllll}3.1 & 2.00000 & -20.49886 & 21 \mathrm{~s} & 1.00091\end{array}$

$\begin{array}{lllll}4.1 & 2.00000 & -19.25991 & 11 \mathrm{~s} & 0.97311\end{array}$

$\begin{array}{lllllll}5.1 & 2.00000 & -15.68559 & 12 \mathrm{py} & 0.78889 & 12 \mathrm{pz} & -0.61451\end{array}$

$\begin{array}{lllllll}6.1 & 2.00000 & -15.68337 & 12 \mathrm{py} & 0.61447 & 12 \mathrm{pz} & 0.78886\end{array}$

$\begin{array}{lllll}7.1 & 2.00000 & -2.58764 & 11 \mathrm{~s} & 1.00079\end{array}$

$\begin{array}{llllllll}8.1 & 2.00000 & -1.61275 & 12 \mathrm{py} & 0.43203 & 12 \mathrm{pz} & 0.81436\end{array}$

$\begin{array}{lllllll}9.1 & 2.00000 & -1.58371 & 12 \mathrm{py} & 0.86619 & 12 \mathrm{pz} & -0.40583\end{array}$

$\begin{array}{lllll}10.1 & 2.00000 & -1.39879 & 31 \mathrm{~s} & 0.90758\end{array}$

$\begin{array}{lllll}11.1 & 2.00000 & -1.09006 & 21 \mathrm{~s} & 0.90178\end{array}$

$\begin{array}{lllllll}12.1 & 1.98016 & -0.66914 & 32 \mathrm{py} & -0.52641 & 32 \mathrm{pz} & 0.70270\end{array}$

$\begin{array}{lllllll}13.1 & 1.97921 & -0.57096 & 32 \mathrm{py} & 0.72717 & 32 \mathrm{pz} & 0.54581\end{array}$

$\begin{array}{lllllllllll}14.1 & 1.96052 & -0.44266 & 1 & 1 \mathrm{~s} & -0.26379 & 12 \mathrm{pz} & 0.45537 & 13 \mathrm{~d} 0 & -0.29043 & 22 \mathrm{py}\end{array}$

$\begin{array}{lll}0.78879 & 22 \mathrm{pz} & 0.25390\end{array}$

$\begin{array}{llllllll}15.1 & 1.95439 & -0.41517 & 12 \text { py } & -0.32628 & 13 \mathrm{~d} 1-0.30847 & 22 \mathrm{pz} & -0.77746\end{array}$

$\begin{array}{llllllllll}16.1 & 0.04604 & 0.51533 & 12 p y & 0.66598 & 12 p y & 0.35621 & 13 \mathrm{~d} 2+0.38821 & 13 \mathrm{~d} 1--\end{array}$ $0.55276 \quad 22$ py 0.36859

$$
22 \mathrm{pz}-1.01029 \quad 22 \mathrm{pz} \quad 0.58930
$$

$\begin{array}{lllllllllll}17.1 & 0.04117 & 0.62073 & 11 \mathrm{~s} & 0.25907 & 12 \mathrm{py} & -0.32100 & 12 \mathrm{pz} & -1.18935 & 12 \mathrm{py}-\end{array}$ $0.26523 \quad 12 \mathrm{pz}-0.61150$

$21 \mathrm{~s}-0.34724$

$$
12 \mathrm{pz}-0.36726 \quad 13 \mathrm{~d} 0 \quad 0.60339 \quad 13 \mathrm{~d} 1-0.42695 \quad 21 \mathrm{~s} \quad-0.34938
$$

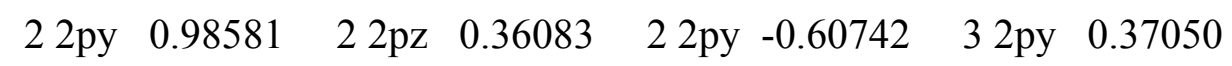

$\begin{array}{llllllllllll}18.1 & 0.01993 & 1.10777 & 12 \mathrm{py} & 0.35613 & 12 \mathrm{pz} & 0.39846 & 3 & 1 \mathrm{~s} & 0.49803 & 3 & 1 \mathrm{~s}\end{array}$ $0.41199 \quad 32$ py 0.29993

$$
\begin{array}{llllll}
32 \mathrm{pz} & 1.20648 & 32 \mathrm{py} & -0.29437 & 32 \mathrm{pz} & -1.24220
\end{array}
$$

\begin{tabular}{|c|c|c|c|c|c|c|c|}
\hline & & $32 \mathrm{py}$ & 1.16667 & 0.29128 & 1.23337 & $32 \mathrm{pz}$ & -0.32828 \\
\hline 1.2 & 2.00000 & -15.68509 & $12 \mathrm{px} \quad 0.99997$ & & & & \\
\hline 2.2 & 2.00000 & -1.57682 & $12 \mathrm{px} \quad 0.98931$ & & & & \\
\hline 3.2 & 1.97831 & -0.56728 & $32 \mathrm{px} \quad 0.90626$ & & & & \\
\hline 4.2 & 1.95408 & -0.40698 & $13 \mathrm{~d} 1+0.26376$ & $22 \mathrm{px} \quad 0.79513$ & & & \\
\hline 5.2 & 0.04624 & 0.48500 & $12 \mathrm{px}-0.52927$ & $13 \mathrm{~d} 2-0.37803$ & $13 \mathrm{~d} 1+$ & 0.55795 & $22 p x-$ \\
\hline 1.08093 & $22 p x$ & 0.61852 & & & & & \\
\hline 6.2 & 0.02150 & 1.00500 & $32 \mathrm{px} \quad 1.24700$ & $32 \mathrm{px}-1.21515$ & & & \\
\hline
\end{tabular}

$\begin{array}{llllllllll}19.1 & 0.01845 & 1.17630 & 12 \mathrm{pz} & -0.25824 & 22 \mathrm{py} & 0.37364 & 22 \mathrm{py} & -0.33356 & 31 \mathrm{~s}\end{array}$ $0.52097 \quad 31 \mathrm{~s} \quad-0.43681$ 


\section{${ }^{2} \mathrm{OTiF}$}

Orbital Occupation Energy Coefficients

$\begin{array}{llllll}1.1 & 2.00000 & -184.40637 & 1 & 1 \mathrm{~s} & 0.97461\end{array}$

$\begin{array}{lllll}2.1 & 2.00000 & -26.25897 & 31 \mathrm{~s} & 1.00064\end{array}$

$\begin{array}{lllll}3.1 & 2.00000 & -21.66463 & 11 \mathrm{~s} & 0.97446\end{array}$

$\begin{array}{lllll}4.1 & 2.00000 & -20.53540 & 21 \mathrm{~s} & 1.00094\end{array}$

$\begin{array}{lllllll}5.1 & 2.00000 & -17.83733 & 12 \mathrm{py} & 0.38089 & 12 \mathrm{pz} & 0.92455\end{array}$

$\begin{array}{lllllll}6.1 & 2.00000 & -17.83122 & 12 \mathrm{py} & 0.92458 & 12 \mathrm{pz} & -0.38095\end{array}$

$\begin{array}{llllll}7.1 & 2.00000 & -2.90742 & 1 & 1 \mathrm{~s} & 1.00260\end{array}$

$\begin{array}{lllllll}8.1 & 2.00000 & -1.83622 & 12 \mathrm{py} & -0.58856 & 12 \mathrm{pz} & -0.75687\end{array}$

$\begin{array}{llllllll}9.1 & 2.00000 & -1.80616 & 12 \mathrm{py} & 0.78088 & 12 \mathrm{pz} & -0.57799\end{array}$

$\begin{array}{llllll}10.1 & 2.00000 & -1.40384 & 31 \mathrm{~s} & 0.92884\end{array}$

$\begin{array}{lllll}11.1 & 2.00000 & -1.14427 & 21 \mathrm{~s} & 0.92014\end{array}$

$\begin{array}{lllllll}12.1 & 1.99639 & -0.58271 & 32 \mathrm{py} & 0.85628 & 32 \mathrm{pz} & 0.33460\end{array}$

$\begin{array}{lllllllll}13.1 & 1.98076 & -0.67191 & 12 \mathrm{pz} & -0.29760 & 32 \mathrm{py} & -0.31523 & 32 \mathrm{pz} & 0.81419\end{array}$

$\begin{array}{lllllllllll}14.1 & 1.95507 & -0.45833 & 12 \mathrm{pz} & 0.51081 & 12 \mathrm{pz} & -0.25760 & 13 \mathrm{~d} 0 & 0.30440 & 22 \mathrm{py} & -\end{array}$ 0.81356

$\begin{array}{llllllllll}15.1 & 1.94712 & -0.46820 & 12 \mathrm{py} & -0.47399 & 12 \mathrm{pz} & -0.44323 & 12 \mathrm{py} & 0.28630 & 12 \mathrm{pz}\end{array}$ $0.28585 \quad 13 \mathrm{~d} 1--0.38257$

\section{$22 \mathrm{pz} \quad 0.80331$}

$\begin{array}{lllllllll}16.1 & 1.00067 & -0.07095 & 11 \mathrm{~s} & \mathbf{0 . 3 6 4 7 7} & \mathbf{1} 3 \mathrm{~d} 0 & -0.31195 & 13 \mathrm{~d} 2+\mathbf{0 . 8 6 6 9 3}\end{array}$

$\begin{array}{lllllllllll}17.1 & 0.05325 & 0.46813 & 11 \mathrm{~s} & 0.29927 & 12 \mathrm{py} & 0.77394 & 12 \mathrm{pz} & 0.50760 & 12 \mathrm{py} & -\end{array}$ $0.45666 \quad 12 \mathrm{pz}-0.42629$

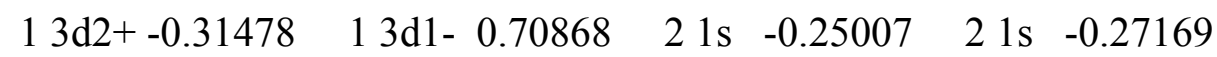

$22 \mathrm{pz} \quad 0.98864$

$$
22 \mathrm{pz}-0.52144
$$

$\begin{array}{lllllllllll}18.1 & 0.04811 & 0.46520 & 12 \mathrm{py} & -0.37781 & 12 \mathrm{pz} & -1.22215 & 12 \mathrm{py} & 0.37900 & 12 \mathrm{pz}\end{array}$ $0.65089 \quad 12 \mathrm{pz} \quad 0.36238$

$13 \mathrm{~d} 0-0.66673 \quad 13 \mathrm{~d} 1--0.32064 \quad 22$ py $-0.96715 \quad 22$ py $\quad 0.51932$

32 py -0.33934

$\begin{array}{llllllllll}19.1 & 0.01840 & 1.06396 & 11 \mathrm{~s} & -0.34503 & 12 \mathrm{py} & -0.33459 & 12 \mathrm{pz} & 0.28797 & 31 \mathrm{~s}\end{array}$ $0.71060 \quad 31 \mathrm{~s} \quad-0.55691$

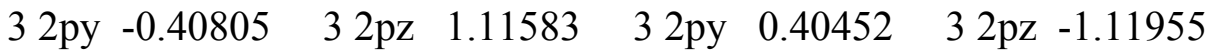

$\begin{array}{llllll}1.2 & 2.00000 & -17.83108 & 12 \mathrm{px} & 0.99998\end{array}$

$\begin{array}{lllll}2.2 & 2.00000 & -1.79455 & 12 \mathrm{px} & 0.99001\end{array}$

$\begin{array}{lllll}3.2 & 1.97979 & -0.56523 & 32 \mathrm{px} & 0.90497\end{array}$

$\begin{array}{llllll}4.2 & 1.94820 & -0.44114 & 13 \mathrm{~d} 1+0.25900 & 22 \mathrm{px} & 0.80194\end{array}$

$\begin{array}{llllllll}5.2 & 0.05211 & 0.41312 & 13 \mathrm{~d} 2-0.46850 & 13 \mathrm{~d} 1+0.57036 & 22 \mathrm{px} & -1.01177 & 22 \mathrm{px}\end{array}$ 0.53225

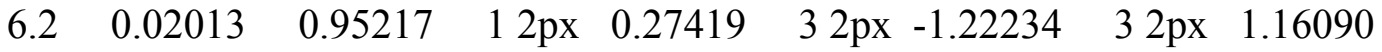




\begin{tabular}{|c|c|c|c|c|c|}
\hline \multicolumn{6}{|l|}{${ }^{3} \mathrm{OVF}$} \\
\hline Orbital & Occupation Energy & Coefficients & & & \\
\hline 1.1 & $2.00000-202.85214$ & $11 \mathrm{~s} \quad 0.97735$ & & & \\
\hline 2.1 & $2.00000 \quad-26.24489$ & $31 \mathrm{~s} \quad 1.00073$ & & & \\
\hline 3.1 & $2.00000-24.14854$ & $11 \mathrm{~s} \quad 0.97686$ & & & \\
\hline 4.1 & $2.00000-20.56653$ & $21 \mathrm{~s} \quad 1.00111$ & & & \\
\hline 5.1 & $2.00000-20.04757$ & 12 py 0.86493 & $12 \mathrm{pz}-0.50174$ & & \\
\hline 6.1 & $2.00000-20.02206$ & $5 \quad 12$ py $\quad 0.50145$ & $12 \mathrm{pz} \quad 0.86395$ & & \\
\hline 7.1 & $2.00000 \quad-3.20990$ & $11 \mathrm{~s} \quad 0.99730$ & & & \\
\hline 8.1 & $2.00000 \quad-1.98739$ & 12 py $\quad 0.89194$ & $12 \mathrm{pz}-0.33279$ & & \\
\hline 9.1 & $2.00000 \quad-1.33107$ & $31 \mathrm{~s} \quad 0.88328$ & $32 \mathrm{pz}-0.34628$ & & \\
\hline 10.1 & $2.00000-1.25027$ & $7 \quad 21 \mathrm{~s} \quad 0.92200$ & & & \\
\hline 11.1 & $2.00000 \quad-0.59706$ & 32 py 0.90355 & & & \\
\hline 12.1 & $1.99329-1.98160$ & 12 py -0.40209 & $12 \mathrm{pz}-0.86988$ & $12 \mathrm{pz} \quad 0.29782$ & \\
\hline 13.1 & $\begin{array}{ll}1.98869 & -0.78966\end{array}$ & $12 \mathrm{pz} \quad 0.31184$ & $12 \mathrm{pz}-0.33814$ & $31 \mathrm{~s} \quad 0.28932$ & $32 \mathrm{pz}$ \\
\hline \multicolumn{6}{|l|}{0.80785} \\
\hline 14.1 & $1.94218 \quad-0.48414$ & $12 \mathrm{pz}-0.38736$ & $13 \mathrm{~d} 0-0.31143$ & 22 py $\quad 0.81361$ & \\
\hline 15.1 & $\begin{array}{ll}1.93260 & -0.50119\end{array}$ & 12 py -0.26146 & $12 \mathrm{pz}-0.67138$ & $\begin{array}{lll}32 \mathrm{pz} & 0.42498\end{array}$ & $8 \quad 13 \mathrm{~d} 1--$ \\
\hline 0.37805 & $22 \mathrm{pz} \quad 0.82636$ & & & & \\
\hline 16.1 & $\begin{array}{ll}1.00023 & -0.06772\end{array}$ & $13 \mathrm{~d} 2+-0.97290$ & & & \\
\hline 17.1 & $0.07111 \quad 0.39385$ & $11 \mathrm{~s}-0.28237$ & 12 py -0.44920 & $12 \mathrm{pz}-1.65131$ & $12 \mathrm{pz}$ \\
\hline \multirow[t]{2}{*}{1.01526} & $5 \quad 12 \mathrm{pz} \quad 0.44534$ & & & & \\
\hline & $13 \mathrm{~d} 0$ & $-0.43895 \quad 13 \mathrm{~d} 1-$ & -0.75939 & $0.43425 \quad 22 \mathrm{pz}-$ & -0.82856 \\
\hline \multicolumn{6}{|c|}{$22 \mathrm{pz} \quad 0.29530$} \\
\hline 18.1 & $0.06129 \quad 0.34232$ & $12 \mathrm{pz} \quad 1.85898$ & $12 \mathrm{pz}-1.01115$ & $12 \mathrm{pz}-0.54977$ & $13 \mathrm{~d} 0$ \\
\hline \multicolumn{6}{|l|}{0.74500} \\
\hline & $21 \mathrm{~s}-$ & -0.25865 & $0.82426 \quad 22$ py -( & $-0.32362 \quad 31 \mathrm{~s} \quad 0$ & 0.29244 \\
\hline 19.1 & 1.13527 & $12 \mathrm{pz} 1.43150$ & 12 py -0.30306 & $12 \mathrm{pz}-0.70175$ & $5 \quad 12 \mathrm{pz}$ \\
\hline \multirow[t]{2}{*}{0.4760} & $\begin{array}{lll}31 \mathrm{~s} & 0.98747\end{array}$ & & & & \\
\hline & $31 \mathrm{~s}-$ & -0.82723 & $1.11180 \quad 32 \mathrm{pz}-1$ & -1.11482 & \\
\hline 1.2 & $2.00000-20.00079$ & $12 \mathrm{px} \quad 0.99898$ & & & \\
\hline 2.2 & $2.00000 \quad-0.58116$ & $32 \mathrm{px} \quad 0.91277$ & & & \\
\hline 3.2 & $\begin{array}{ll}1.99541 & -2.04527\end{array}$ & $12 \mathrm{px}-0.99121$ & & & \\
\hline 4.2 & $1.93366 \quad-0.46387$ & $13 \mathrm{~d} 1+0.32752$ & $22 p x \quad 0.80065$ & & \\
\hline 5.2 & $\begin{array}{ll}0.99978 & -0.06708\end{array}$ & $13 \mathrm{~d} 2-0.95704$ & $13 \mathrm{~d} 1+-0.29268$ & & \\
\hline 6.2 & $0.07172 \quad 0.28938$ & $13 \mathrm{~d} 1+-0.80996$ & $22 \mathrm{px} \quad 0.78764$ & $22 \mathrm{px}-0.28241$ & \\
\hline
\end{tabular}




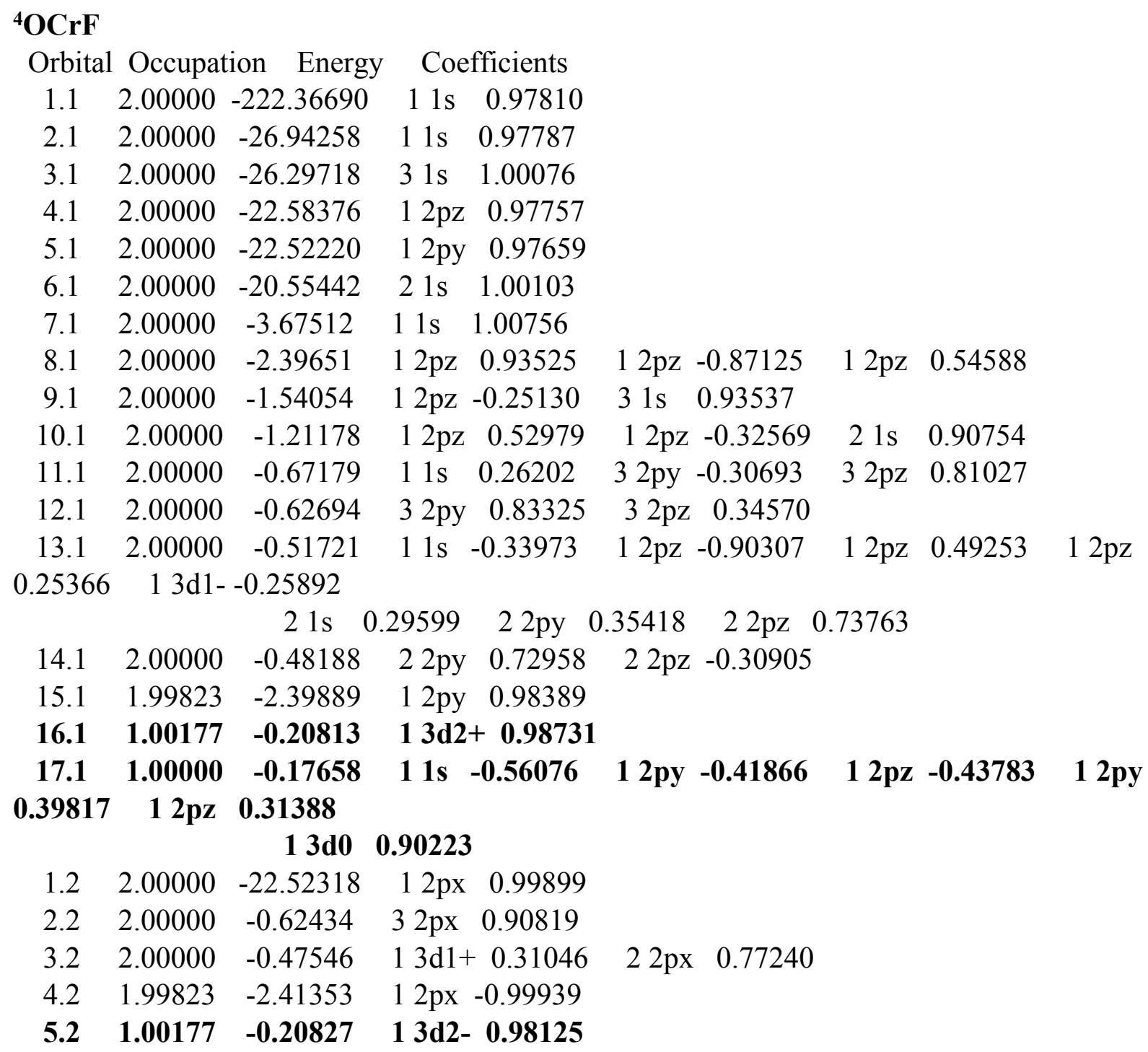




\begin{tabular}{|c|c|c|c|c|c|c|}
\hline \multicolumn{7}{|l|}{${ }^{5} \mathrm{OMnF}$} \\
\hline Orbital & Occupatio & on Energy & Coefficients & & & \\
\hline 1.1 & 2.00000 & -242.64778 & $11 \mathrm{~s} \quad 0.98900$ & & & \\
\hline 2.1 & 2.00000 & -29.70697 & $11 \mathrm{~s} \quad 0.98895$ & & & \\
\hline 3.1 & 2.00000 & -26.28649 & $31 \mathrm{~s} \quad 1.00066$ & & & \\
\hline 4.1 & 2.00000 & -25.06951 & 12 py 0.90727 & $12 \mathrm{pz} \quad 0.42044$ & & \\
\hline 5.1 & 2.00000 & -25.06236 & 12 py 0.42044 & $12 \mathrm{pz}-0.90728$ & & \\
\hline 6.1 & 2.00000 & -20.55367 & $21 \mathrm{~s} \quad 1.00092$ & & & \\
\hline 7.1 & 2.00000 & -4.02998 & $11 \mathrm{~s} \quad 1.00993$ & & & \\
\hline 8.1 & 2.00000 & -2.64004 & 12 py 0.97253 & & & \\
\hline 9.1 & 2.00000 & -2.63600 & $12 \mathrm{pz}-0.95635$ & & & \\
\hline 10.1 & 2.00000 & -1.52269 & $31 \mathrm{~s} \quad 0.94123$ & & & \\
\hline 11.1 & 2.00000 & -1.18636 & $21 \mathrm{~s} \quad 0.91741$ & & & \\
\hline 12.1 & 2.00000 & -0.64556 & $12 \mathrm{pz}-0.38178$ & $12 \mathrm{pz}-0.33616$ & 32 ру -0.42332 & $32 \mathrm{pz}$ \\
\hline \multicolumn{7}{|l|}{0.76450} \\
\hline 13.1 & 2.00000 & -0.60995 & 32 py 0.79226 & $32 \mathrm{pz} \quad 0.43969$ & & \\
\hline 14.1 & 2.00000 & -0.49030 & $11 \mathrm{~s} \quad-0.38885$ & $12 \mathrm{pz}-0.28614$ & $12 \mathrm{pz}-0.33976$ & $13 \mathrm{~d} 1--$ \\
\hline \multirow[t]{2}{*}{0.27986} & $22 p y$ & 0.59735 & & & & \\
\hline & & $22 \mathrm{pz}$ & 0.53992 & & & \\
\hline 15.1 & 2.00000 & -0.42729 & $13 \mathrm{~d} 1-0.38828$ & 2 2py 0.55384 & $22 \mathrm{pz}-0.45058$ & \\
\hline 16.1 & 1.00000 & -0.22300 & $13 \mathrm{~d} 0 \quad 0.94173$ & & & \\
\hline 17.1 & 1.00000 & -0.26078 & $13 \mathrm{~d} 2+0.94249$ & $13 d 1-0.26201$ & & \\
\hline 1.2 & 2.00000 & -25.05816 & $12 \mathrm{px} \quad 0.99996$ & & & \\
\hline 2.2 & 2.00000 & -2.63300 & $12 \mathrm{px} \quad 1.00090$ & & & \\
\hline 3.2 & 2.00000 & -0.61253 & $32 \mathrm{px} \quad 0.91995$ & & & \\
\hline 4.2 & 2.00000 & -0.46398 & $22 \mathrm{px}-0.85132$ & & & \\
\hline 5.2 & 1.00000 & -0.22551 & $13 \mathrm{~d} 1+-0.96905$ & & & \\
\hline 6.2 & 1.00000 & -0.26028 & $13 \mathrm{~d} 2-0.97569$ & & & \\
\hline
\end{tabular}




\section{${ }^{6} \mathrm{OFeF}$}

\begin{tabular}{|c|c|c|c|c|c|c|}
\hline Orbital & 1 Occupat & tion Energy & Coefficients & & & \\
\hline 1.1 & 2.00000 & -263.88525 & $11 \mathrm{~s} \quad 0.98435$ & & & \\
\hline 2.1 & 2.00000 & -32.67642 & $11 \mathrm{~s} \quad 0.98453$ & & & \\
\hline 3.1 & 2.00000 & -27.73901 & $12 \mathrm{pz} \quad 0.99997$ & & & \\
\hline 4.1 & 2.00000 & -26.31176 & $31 \mathrm{~s} \quad 1.00071$ & & & \\
\hline 5.1 & 2.00000 & -20.52602 & $2 \mathrm{ls} \quad 1.00098$ & & & \\
\hline 6.1 & 2.00000 & -4.47482 & $11 \mathrm{~s} \quad 1.01276$ & & & \\
\hline 7.1 & 2.00000 & -2.97285 & $12 \mathrm{pz} \quad 0.98417$ & & & \\
\hline 8.1 & 2.00000 & -1.55455 & $31 \mathrm{~s} \quad 0.93583$ & & & \\
\hline 9.1 & 2.00000 & -1.19511 & $21 \mathrm{~s} \quad 0.89589$ & & & \\
\hline 10.1 & 2.00000 & -0.68994 & $11 \mathrm{~s} \quad 0.30745$ & $\begin{array}{lll}32 \mathrm{pz} & 0.83999\end{array}$ & & \\
\hline 11.1 & 2.00000 & -0.50300 & $11 \mathrm{~s} \quad-0.39733$ & $13 \mathrm{~d} 0-0.26540$ & $22 \mathrm{pz} \quad 0.79091$ & $32 \mathrm{pz}$ \\
\hline 0.28777 & & & & & & \\
\hline 12.1 & 1.00000 & -0.30000 & $11 \mathrm{~s} \quad-0.28771$ & $13 \mathrm{~d} 0 \quad 0.93444$ & & \\
\hline 13.1 & 1.00000 & -0.38089 & $13 \mathrm{~d} 2+1.00184$ & & & \\
\hline 1.2 & 2.00000 & -27.74466 & $12 \mathrm{px} \quad 0.99997$ & & & \\
\hline 2.2 & 2.00000 & -2.97881 & $12 \mathrm{px} \quad 1.00100$ & & & \\
\hline 3.2 & 2.00000 & -0.64344 & $32 \mathrm{px} \quad 0.90681$ & & & \\
\hline 4.2 & 2.00000 & -0.48108 & $13 \mathrm{~d} 1+-0.31301$ & $22 p x-0.76175$ & & \\
\hline 5.2 & 1.00000 & -0.30686 & $13 \mathrm{~d} 1+0.95497$ & $22 p x-0.31847$ & & \\
\hline 1.3 & 2.00000 & -27.74466 & 12 py 0.99997 & & & \\
\hline 2.3 & 2.00000 & -2.97881 & 12 py 1.00100 & & & \\
\hline 3.3 & 2.00000 & -0.64344 & 32 py $\quad 0.90681$ & & & \\
\hline 4.3 & 2.00000 & -0.48108 & $13 \mathrm{~d} 1--0.31301$ & 2 2py -0.76175 & & \\
\hline 5.3 & 1.00000 & -0.30686 & $13 d 1-0.95497$ & 2 2py -0.31847 & & \\
\hline 1.4 & 1.00000 & -0.38089 & $13 \mathrm{~d} 2-1.00184$ & & & \\
\hline
\end{tabular}




\begin{tabular}{|c|c|c|c|c|c|}
\hline \multicolumn{6}{|c|}{${ }^{5} \mathrm{OCoF}$} \\
\hline Orbital & 1 Occupat & tion Energy & Coefficients & & \\
\hline 1.1 & 2.00000 & -285.79216 & $11 \mathrm{~s} \quad 0.98322$ & & \\
\hline 2.1 & 2.00000 & -35.49969 & $11 \mathrm{~s} \quad 0.98324$ & & \\
\hline 3.1 & 2.00000 & -30.27383 & $12 \mathrm{pz} \quad 0.99996$ & & \\
\hline 4.1 & 2.00000 & -26.25551 & $31 \mathrm{~s} \quad 1.00070$ & & \\
\hline 5.1 & 2.00000 & -20.59755 & $21 \mathrm{~s} \quad 1.00100$ & & \\
\hline 6.1 & 2.00000 & -4.65974 & $11 \mathrm{~s} \quad 1.00161$ & & \\
\hline 7.1 & 2.00000 & -3.07613 & $12 \mathrm{pz} \quad 0.99237$ & & \\
\hline 8.1 & 2.00000 & -1.49887 & $31 \mathrm{~s} \quad 0.93681$ & & \\
\hline 9.1 & 2.00000 & -1.25181 & $21 \mathrm{~s} \quad 0.92826$ & & \\
\hline 10.1 & 2.00000 & -0.65377 & $13 \mathrm{~d} 2+1.00576$ & & \\
\hline 11.1 & 2.00000 & -0.61933 & $32 \mathrm{pz}-0.88969$ & & \\
\hline 12.1 & 1.91021 & -0.56339 & $13 \mathrm{~d} 0-0.41575$ & $22 \mathrm{pz} \quad 0.82576$ & \\
\hline 13.1 & 1.08966 & -0.23139 & $11 \mathrm{~s} \quad-0.38035$ & $13 \mathrm{~d} 0 \quad 0.89093$ & $22 \mathrm{pz} \quad 0.35566$ \\
\hline 1.2 & 2.00000 & -30.26926 & $12 \mathrm{px} \quad 0.99997$ & & \\
\hline 2.2 & 2.00000 & -3.06889 & $12 \mathrm{px} \quad 0.99973$ & & \\
\hline 3.2 & 2.00000 & -0.58596 & $32 \mathrm{px} \quad 0.91681$ & & \\
\hline 4.2 & 1.85225 & -0.52771 & $13 \mathrm{~d} 1+-0.57405$ & $22 p x-0.69337$ & \\
\hline 5.2 & 1.14775 & -0.26557 & $13 \mathrm{~d} 1+0.83954$ & $22 \mathrm{px}-0.58829$ & \\
\hline 1.3 & 2.00000 & -30.26926 & 12 py 0.99997 & & \\
\hline 2.3 & 2.00000 & -3.06889 & 12 py 0.99973 & & \\
\hline 3.3 & 2.00000 & -0.58596 & 32 py 0.91681 & & \\
\hline 4.3 & 1.85225 & -0.52771 & 1 3d1- -0.57405 & 2 2py -0.69337 & \\
\hline 5.3 & 1.14775 & -0.26557 & $13 \mathrm{~d} 1-0.83954$ & 2 2py -0.58829 & \\
\hline 1.4 & 1.00013 & -0.22763 & $13 \mathrm{~d} 2-1.00741$ & & \\
\hline
\end{tabular}




\begin{tabular}{|c|c|c|c|c|c|c|}
\hline \multicolumn{7}{|l|}{${ }^{4} \mathrm{ONiF}$} \\
\hline Orbital & 1 Occupa & tion Energy & Coefficients & & & \\
\hline 1.1 & 2.00000 & -308.78239 & $11 \mathrm{~s} \quad 0.99247$ & & & \\
\hline 2.1 & 2.00000 & -38.64299 & $11 \mathrm{~s} \quad 0.99236$ & & & \\
\hline 3.1 & 2.00000 & -33.12068 & $12 \mathrm{pz} \quad 0.99995$ & & & \\
\hline 4.1 & 2.00000 & -26.26099 & $31 \mathrm{~s} \quad 1.00071$ & & & \\
\hline 5.1 & 2.00000 & -20.61073 & $21 \mathrm{~s} \quad 1.00103$ & & & \\
\hline 6.1 & 2.00000 & -5.03201 & $11 \mathrm{~s} \quad 1.00451$ & & & \\
\hline 7.1 & 2.00000 & -3.35205 & $12 \mathrm{pz} \quad 0.99351$ & & & \\
\hline 8.1 & 2.00000 & -1.50744 & $31 \mathrm{~s} \quad 0.93605$ & & & \\
\hline 9.1 & 2.00000 & -1.27012 & 2 1s $\quad 0.92652$ & & & \\
\hline 10.1 & 2.00000 & -0.66859 & $13 \mathrm{~d} 2+1.01206$ & & & \\
\hline 11.1 & 2.00000 & -0.63120 & $32 \mathrm{pz} \quad 0.88757$ & & & \\
\hline 12.1 & 1.90992 & -0.58966 & 1 3d0 -0.42744 & $22 \mathrm{pz} \quad 0.82220$ & & \\
\hline 13.1 & 1.13241 & -0.28574 & $11 \mathrm{~s} \quad-0.32917$ & $13 \mathrm{~d} 0 \quad 0.89870$ & $22 \mathrm{pz}$ & 0.36534 \\
\hline 1.2 & 2.00000 & -33.09809 & $12 \mathrm{px} \quad 0.99995$ & & & \\
\hline 2.2 & 2.00000 & -3.33267 & $12 \mathrm{px} \quad 0.99971$ & & & \\
\hline 3.2 & 2.00000 & -0.59308 & $32 \mathrm{px} \quad 0.91765$ & & & \\
\hline 4.2 & 1.82320 & -0.54892 & $13 \mathrm{~d} 1+-0.59964$ & $22 p x-0.68784$ & & \\
\hline 5.2 & 1.17680 & -0.30215 & $13 \mathrm{~d} 1+0.82373$ & $22 p x-0.60437$ & & \\
\hline 1.3 & 2.00000 & -33.09809 & 1 2py 0.99995 & & & \\
\hline 2.3 & 2.00000 & -3.33267 & 12 py 0.99971 & & & \\
\hline 3.3 & 2.00000 & -0.59308 & 32 py $\quad 0.91765$ & & & \\
\hline 4.3 & 1.82320 & -0.54892 & $13 d 1--0.59964$ & 2 2ру -0.68784 & & \\
\hline 5.3 & 1.17680 & -0.30215 & $13 \mathrm{~d} 1-0.82373$ & 22 py -0.60437 & & \\
\hline 1.4 & 1.95768 & -0.64996 & $13 \mathrm{~d} 2-1.01201$ & & & \\
\hline
\end{tabular}




\begin{tabular}{|c|c|c|c|c|c|}
\hline \multicolumn{6}{|c|}{${ }^{3} \mathrm{OCuF}$} \\
\hline Orbital & 1 Occupat & tion Energy & Coefficients & & \\
\hline 1.1 & 2.00000 & -332.73651 & $11 \mathrm{~s} \quad 0.97828$ & & \\
\hline 2.1 & 2.00000 & -41.96465 & $11 \mathrm{~s} \quad 0.97827$ & & \\
\hline 3.1 & 2.00000 & -36.10924 & $12 \mathrm{pz} \quad 0.99994$ & & \\
\hline 4.1 & 2.00000 & -26.27016 & $31 \mathrm{~s} \quad 1.00076$ & & \\
\hline 5.1 & 2.00000 & -20.65020 & $21 \mathrm{~s} \quad 1.00110$ & & \\
\hline 6.1 & 2.00000 & -5.44188 & $11 \mathrm{~s} \quad 0.99947$ & & \\
\hline 7.1 & 2.00000 & -3.58792 & $12 \mathrm{pz} \quad 0.97412$ & & \\
\hline 8.1 & 2.00000 & -1.45014 & $31 \mathrm{~s} \quad 0.93989$ & & \\
\hline 9.1 & 2.00000 & -1.19542 & $21 \mathrm{~s} \quad 0.93355$ & & \\
\hline 10.1 & 1.99960 & -0.75776 & $32 \mathrm{pz} \quad 0.84847$ & & \\
\hline 11.1 & 1.99924 & -0.76083 & $13 \mathrm{~d} 2+1.00938$ & & \\
\hline 12.1 & 1.98810 & -0.62047 & $11 \mathrm{~s} \quad-0.50500$ & $13 \mathrm{~d} 0 \quad 0.70930$ & $22 \mathrm{pz} \quad 0.50529$ \\
\hline 13.1 & 1.97547 & -0.77127 & $13 \mathrm{~d} 0-\mathbf{0 . 6 6 5 0 0}$ & $22 \mathrm{pz} \quad 0.66251$ & \\
\hline 1.2 & 2.00000 & -36.10520 & $12 \mathrm{px} \quad 0.99997$ & & \\
\hline 2.2 & 2.00000 & -3.63119 & $12 \mathrm{px} \quad 0.99729$ & & \\
\hline 3.2 & 1.99961 & -0.61052 & $32 \mathrm{px} \quad 0.91590$ & & \\
\hline 4.2 & 1.82802 & -0.59772 & $13 \mathrm{~d} 1+-0.73750$ & $22 p x-0.57909$ & \\
\hline 5.2 & 1.17243 & -0.34553 & $13 \mathrm{~d} 1+-0.69625$ & $22 p x \quad 0.73440$ & \\
\hline 1.3 & 2.00000 & -36.10520 & 12 py 0.99997 & & \\
\hline 2.3 & 2.00000 & -3.63119 & 12 py 0.99729 & & \\
\hline 3.3 & 1.99961 & -0.61052 & 3 2ру 0.91590 & & \\
\hline 4.3 & 1.82802 & -0.59772 & $13 \mathrm{dd1}-\mathbf{- 0 . 7 3 7 5 0}$ & 2 2py -0.57909 & \\
\hline 5.3 & 1.17243 & -0.34553 & $13 d 1--0.69625$ & 2 2py 0.73440 & \\
\hline 1.4 & 1.99924 & $-\mathbf{- 0 . 7 6 0 8 3}$ & $13 \mathrm{~d} 2-1.00938$ & & \\
\hline
\end{tabular}




\begin{tabular}{|c|c|c|c|c|c|c|c|c|}
\hline \\
\hline \multicolumn{9}{|c|}{ Orbital $\mathrm{O}$} \\
\hline 1.1 & 2.00000 & -166.94423 & $11 \mathrm{~s} \quad 0.97310$ & & & & & \\
\hline 2.1 & 2.00000 & -26.28258 & $31 \mathrm{~s} \quad 1.00062$ & & & & & \\
\hline 3.1 & 2.00000 & -20.63202 & $21 \mathrm{~s} \quad 1.00074$ & & & & & \\
\hline 4.1 & 2.00000 & -19.40819 & $11 \mathrm{~s} \quad 0.97308$ & & & & & \\
\hline 5.1 & 2.00000 & -15.83130 & $12 \mathrm{pz} \quad 0.99999$ & & & & & \\
\hline 6.1 & 2.00000 & -2.71254 & $11 \mathrm{~s} \quad 1.01726$ & & & & & \\
\hline 7.1 & 2.00000 & -1.71269 & $12 \mathrm{pz} \quad 0.90056$ & $12 \mathrm{pz}$ & 0.77249 & $12 \mathrm{pz}$ & 0.47843 & \\
\hline 8.1 & 2.00000 & -1.50921 & $12 \mathrm{pz} \quad 0.25496$ & $12 p z$ & 0.30737 & $31 \mathrm{~s}$ & 0.91985 & \\
\hline 9.1 & 2.00000 & -1.03637 & $12 \mathrm{pz}-0.26626$ & $12 \mathrm{pz}$ & -0.53577 & $12 \mathrm{pz}$ & -0.35285 & $21 \mathrm{~s}$ \\
\hline \multicolumn{9}{|c|}{$z-0.53437$} \\
\hline 10.1 & 2.00000 & -0.63423 & $12 \mathrm{pz} \quad 0.27710$ & $32 \mathrm{pz}$ & 0.68705 & 32 py & -0.57881 & \\
\hline 11.1 & 2.00000 & -0.60810 & $12 \mathrm{pz}-0.36374$ & $32 \mathrm{pz}$ & 0.57918 & 32 py & 0.70366 & \\
\hline 12.1 & 1.97557 & -0.77389 & $12 p z-0.30666$ & $21 \mathrm{~s}$ & -0.64036 & $22 \mathrm{pz}$ & -0.70956 & \\
\hline 1.2 & 2.00000 & -15.83116 & $12 \mathrm{px} \quad 0.99998$ & & & & & \\
\hline 2.2 & 2.00000 & -1.69501 & $12 \mathrm{px} \quad 0.98930$ & $12 \mathrm{px}$ & 0.52438 & $12 \mathrm{px}$ & 0.35885 & \\
\hline 3.2 & 2.00000 & -0.60253 & $32 \mathrm{px} \quad 0.91162$ & & & & & \\
\hline 4.2 & 1.97137 & -0.52657 & $22 \mathrm{px} \quad 0.87393$ & & & & & \\
\hline 1.3 & 2.00000 & -26.28258 & $31 \mathrm{~s} \quad 1.00058$ & & & & & \\
\hline 2.3 & 2.00000 & -15.83146 & 12 py 0.99999 & & & & & \\
\hline 3.3 & 2.00000 & -1.71898 & 12 ру 0.87166 & 12 py & 0.65766 & 12 py & 0.40001 & $31 \mathrm{~s}$ \\
\hline \multicolumn{9}{|l|}{0.31819} \\
\hline 4.3 & 2.00000 & -1.48908 & 12 py 0.40972 & $12 p y$ & 0.45748 & 12 py & 0.31845 & $31 \mathrm{~s}$ \\
\hline \multicolumn{9}{|l|}{0.88869} \\
\hline 5.3 & 2.00000 & -0.62504 & 12 py $\quad 0.50707$ & 12 py & 0.31682 & 32 py & 0.87162 & \\
\hline 6.3 & 2.00000 & -0.59654 & 12 py -0.28370 & $32 \mathrm{pz}$ & 0.89531 & & & \\
\hline 7.3 & 0.99377 & -0.26549 & 2 2py 0.93946 & & & & & \\
\hline 1.4 & 2.00000 & -0.60644 & $32 \mathrm{px} \quad 0.90812$ & & & & & \\
\hline
\end{tabular}




\begin{tabular}{|c|c|c|c|c|c|c|c|}
\hline \multicolumn{8}{|l|}{${ }^{1} \mathrm{OTiF}_{2}$} \\
\hline Orbital & 1 Occupat & tion Energy & Coefficients & & & & \\
\hline 1.1 & 2.00000 & -184.56004 & $11 \mathrm{~s} \quad 0.97461$ & & & & \\
\hline 2.1 & 2.00000 & -26.29750 & $31 \mathrm{~s} \quad 1.00070$ & & & & \\
\hline 3.1 & 2.00000 & -21.83694 & $11 \mathrm{~s} \quad 0.97461$ & & & & \\
\hline 4.1 & 2.00000 & -20.62998 & $21 \mathrm{~s} \quad 1.00094$ & & & & \\
\hline 5.1 & 2.00000 & -18.00020 & $12 \mathrm{pz} \quad 0.99990$ & & & & \\
\hline 6.1 & 2.00000 & -3.05297 & $11 \mathrm{~s} \quad 1.03391$ & & & & \\
\hline 7.1 & 2.00000 & -1.97302 & $12 \mathrm{pz} \quad 0.94901$ & $12 \mathrm{pz}-0.43806$ & $12 \mathrm{pz}$ & 0.28929 & \\
\hline 8.1 & 2.00000 & -1.53934 & $31 \mathrm{~s} \quad 0.93483$ & & & & \\
\hline 9.1 & 2.00000 & -1.23801 & $12 \mathrm{pz}-0.26027$ & $12 \mathrm{pz} \quad 0.30996$ & $21 \mathrm{~s} \quad \mathrm{c}$ & 0.92840 & \\
\hline 10.1 & 2.00000 & -0.66511 & $32 \mathrm{pz}-0.58105$ & 32 py 0.67611 & & & \\
\hline 11.1 & 2.00000 & -0.62704 & $32 \mathrm{pz} \quad 0.68993$ & 3 2py 0.60749 & & & \\
\hline 12.1 & 1.93811 & -0.56423 & $12 \mathrm{pz}-1.16389$ & $12 \mathrm{pz} \quad 0.70944$ & $12 \mathrm{pz}$ & 0.33240 & $13 \mathrm{~d} 0$ \\
\hline 0.49843 & $21 \mathrm{~s}$ & 0.29860 & & & & & \\
\hline & & $22 \mathrm{pz} \quad \mathrm{c}$ & 0.82792 & & & & \\
\hline 1.2 & 2.00000 & -18.00419 & $12 \mathrm{px} \quad 0.99998$ & & & & \\
\hline 2.2 & 2.00000 & -1.93752 & $12 \mathrm{px} \quad 0.98574$ & & & & \\
\hline 3.2 & 2.00000 & -0.61866 & $32 \mathrm{px} \quad 0.91195$ & & & & \\
\hline 4.2 & 1.93153 & -0.52377 & $13 \mathrm{~d} 1+0.39853$ & $22 \mathrm{px} \quad 0.78785$ & & & \\
\hline 1.3 & 2.00000 & -26.29750 & $31 \mathrm{~s} \quad 1.00067$ & & & & \\
\hline 2.3 & 2.00000 & -18.00688 & 12 py 1.00002 & & & & \\
\hline 3.3 & 2.00000 & -1.95077 & 12 py 0.90872 & 12 py -0.72648 & $12 p y$ & 0.41649 & \\
\hline 4.3 & 2.00000 & -1.52051 & 12 py -0.27052 & 12 py 0.48155 & $12 p y$ & -0.33500 & $31 \mathrm{~s}$ \\
\hline 0.92543 & & & & & & & \\
\hline 5.3 & 2.00000 & -0.64514 & 12 py -0.66575 & 12 py 0.41907 & $32 p y$ & 0.90960 & \\
\hline 6.3 & 2.00000 & -0.61600 & 12 py 0.46232 & 12 py -0.26486 & $32 \mathrm{pz}$ & 0.93237 & \\
\hline 7.3 & 1.94814 & -0.53474 & 12 py 0.52190 & 12 py -0.29824 & $13 \mathrm{~d} 1-$ & 0.31225 & 22 py \\
\hline 0.83192 & & & & & & & \\
\hline 1.4 & 2.00000 & -0.62718 & $32 \mathrm{px} \quad 0.90192$ & & & & \\
\hline
\end{tabular}




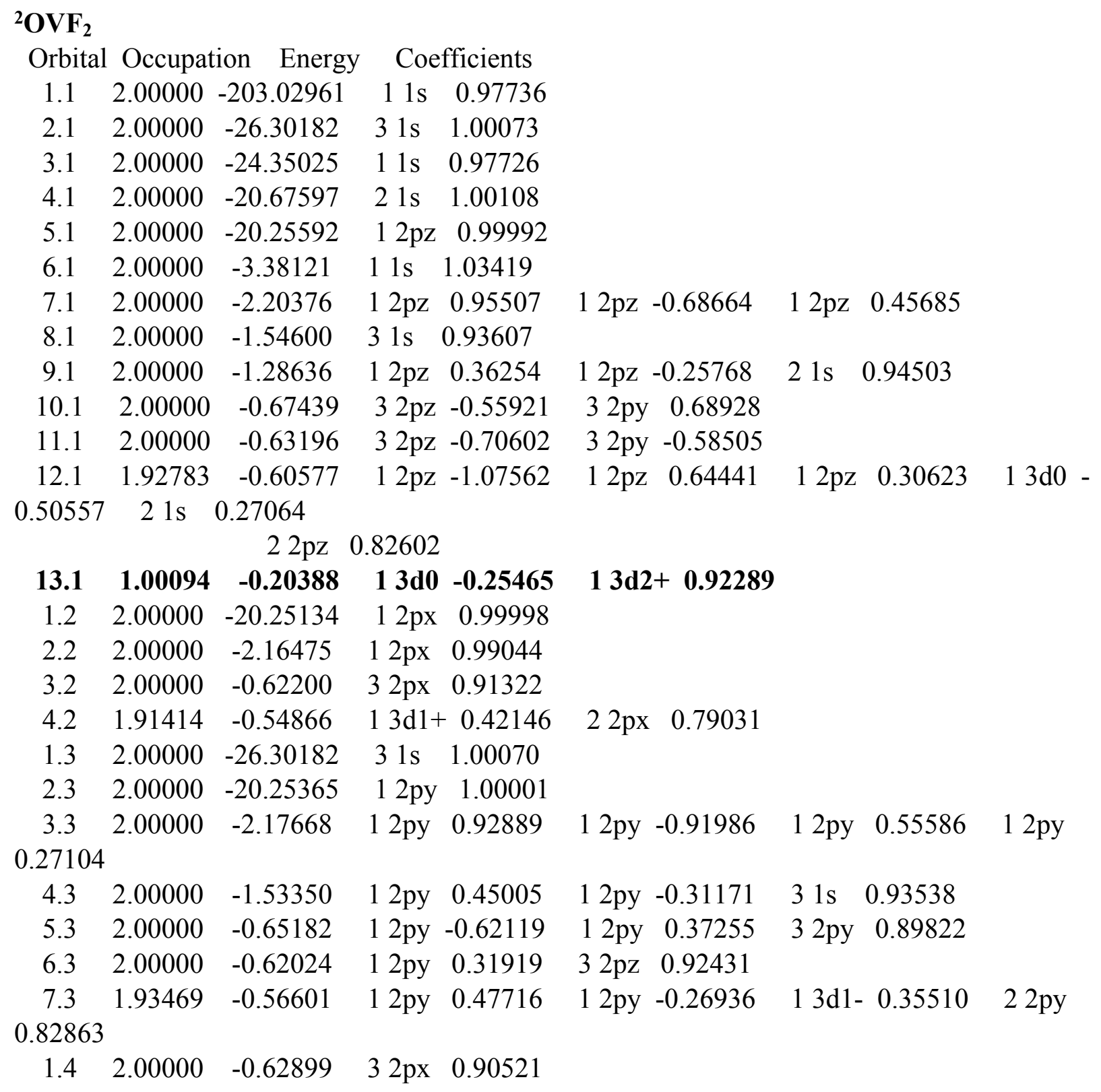




\begin{tabular}{|c|c|c|c|c|c|c|}
\hline \multicolumn{7}{|l|}{${ }^{3} \mathrm{OCrF}_{2}$} \\
\hline \multicolumn{7}{|c|}{ Orbital Oc } \\
\hline 1.1 & 2.00000 & -222.38459 & $11 \mathrm{~s} \quad 0.97810$ & & & \\
\hline 2.1 & 2.00000 & -26.96663 & $11 \mathrm{~s} \quad 0.97775$ & & & \\
\hline 3.1 & 2.00000 & -26.28938 & $31 \mathrm{~s} \quad 1.00074$ & & & \\
\hline 4.1 & 2.00000 & -22.61127 & $12 \mathrm{pz} \quad 0.99993$ & & & \\
\hline 5.1 & 2.00000 & -20.72513 & 2 1s 1.00106 & & & \\
\hline 6.1 & 2.00000 & -3.70024 & $11 \mathrm{~s} \quad 1.01012$ & & & \\
\hline 7.1 & 2.00000 & -2.43140 & $12 \mathrm{pz} \quad 0.96075$ & $12 \mathrm{pz}-0.70340$ & $12 \mathrm{pz} \quad 0.45946$ & \\
\hline 8.1 & 2.00000 & -1.53462 & $31 \mathrm{~s} \quad 0.93481$ & & & \\
\hline 9.1 & 2.00000 & -1.33257 & $12 \mathrm{pz} \quad 0.38927$ & $12 \mathrm{pz}-0.27165$ & $21 \mathrm{~s} \quad 0.95920$ & \\
\hline 10.1 & 2.00000 & -0.65829 & $11 \mathrm{~s} \quad-0.27328$ & $32 \mathrm{pz}-0.54273$ & 32 py $\quad 0.69622$ & \\
\hline 11.1 & 2.00000 & -0.62279 & $32 p z-0.70773$ & 32 py -0.56925 & & \\
\hline 12.1 & 1.91339 & -0.64942 & $12 \mathrm{pz} \quad 1.11829$ & $12 \mathrm{pz} \quad-0.66243$ & $12 \mathrm{pz}-0.30536$ & $13 \mathrm{~d} 0$ \\
\hline \multirow[t]{2}{*}{0.50032} & $21 \mathrm{~s}$ & -0.29326 & & & & \\
\hline & & $22 p z-$ & -0.83264 & & & \\
\hline 13.1 & 0.99575 & -0.20624 & $11 s \quad-0.36142$ & $13 \mathrm{~d} 0 \quad 0.33187$ & $13 \mathrm{~d} 2+-0.90462$ & \\
\hline 1.2 & 2.00000 & -22.59265 & $12 \mathrm{px} \quad 0.99998$ & & & \\
\hline 2.2 & 2.00000 & -2.38777 & $12 \mathrm{px} \quad 0.99485$ & & & \\
\hline 3.2 & 2.00000 & -0.61729 & $32 \mathrm{px} \quad 0.91538$ & & & \\
\hline 4.2 & 1.88332 & -0.57280 & $13 \mathrm{~d} 1+0.45060$ & $22 \mathrm{px} \quad 0.78867$ & & \\
\hline 5.2 & 0.11784 & 0.08905 & $12 \mathrm{px} \quad-0.55350$ & $12 \mathrm{px} \quad 0.58669$ & $13 \mathrm{~d} 1+0.85447$ & $22 \mathrm{px}-$ \\
\hline \multicolumn{7}{|l|}{0.77936} \\
\hline 1.3 & 2.00000 & -26.28938 & $31 \mathrm{~s} \quad 1.00074$ & & & \\
\hline 2.3 & 2.00000 & -22.59666 & 12 py 1.00000 & & & \\
\hline 3.3 & 2.00000 & -2.39421 & 12 py 0.94339 & 12 py -0.72896 & 12 py 0.41765 & \\
\hline 4.3 & 2.00000 & -1.52671 & 12 py 0.40078 & 12 py -0.26820 & $31 \mathrm{~s} \quad 0.93826$ & \\
\hline 5.3 & 2.00000 & -0.64031 & 12 py -0.52927 & 12 py 0.27773 & 32 ру 0.85309 & $32 \mathrm{pz}-$ \\
\hline \multicolumn{7}{|l|}{0.28355} \\
\hline 6.3 & 2.00000 & -0.60498 & 3 2py 0.30072 & $32 \mathrm{pz} \quad 0.88173$ & & \\
\hline 7.3 & 1.91514 & -0.59930 & 12 py 0.44208 & $13 \mathrm{~d} 1-0.39324$ & 2 2py 0.82393 & \\
\hline 8.3 & 0.08627 & 0.15536 & 12 py -1.68603 & 12 py 0.83588 & 12 py 0.54019 & $13 \mathrm{~d} 1--$ \\
\hline \multirow[t]{2}{*}{0.84716} & 2 2py & 0.77341 & & & & \\
\hline & & 22 py - & -0.25183 & 0.46805 & & \\
\hline 1.4 & 2.00000 & -0.62207 & $32 \mathrm{px} \quad 0.91835$ & & & \\
\hline 2.4 & 0.99830 & -0.20239 & $13 \mathrm{~d} 2-1.00078$ & & & \\
\hline
\end{tabular}




\begin{tabular}{|c|c|c|c|c|c|c|c|}
\hline \multicolumn{8}{|c|}{${ }^{4} \mathrm{OMnF}_{2}$} \\
\hline Orbital & 1 Occupat & tion Energy & Coefficients & & & & \\
\hline 1.1 & 2.00000 & -242.65026 & $11 \mathrm{~s} \quad 0.98900$ & & & & \\
\hline 2.1 & 2.00000 & -29.71744 & $11 \mathrm{~s} \quad 0.98871$ & & & & \\
\hline 3.1 & 2.00000 & -26.29131 & $3 \mathrm{ls} \quad 1.00073$ & & & & \\
\hline 4.1 & 2.00000 & -25.08042 & $12 \mathrm{pz} \quad 0.99993$ & & & & \\
\hline 5.1 & 2.00000 & -20.71520 & $21 \mathrm{~s} \quad 1.00114$ & & & & \\
\hline 6.1 & 2.00000 & -4.04207 & $11 \mathrm{~s} \quad 1.01702$ & & & & \\
\hline 7.1 & 2.00000 & -2.66502 & $12 \mathrm{pz} \quad 0.98314$ & & & & \\
\hline 8.1 & 2.00000 & -1.53980 & $31 \mathrm{~s} \quad 0.93537$ & & & & \\
\hline 9.1 & 2.00000 & -1.33923 & $21 \mathrm{~s} \quad 0.96034$ & & & & \\
\hline 10.1 & 2.00000 & -0.66991 & $11 \mathrm{~s} \quad-0.30153$ & $12 \mathrm{pz} \quad 0.37955$ & $12 \mathrm{pz}$ & 0.30083 & $32 \mathrm{pz}$ \\
\hline 0.48109 & 32 py & 0.72857 & & & & & \\
\hline 11.1 & 2.00000 & -0.62406 & $\begin{array}{lll}32 \mathrm{pz} & 0.75268\end{array}$ & 32 py 0.51306 & & & \\
\hline 12.1 & 1.86723 & -0.61407 & $12 \mathrm{pz}-0.26595$ & $12 \mathrm{pz}-0.31754$ & $13 \mathrm{~d} 0$ & -0.55191 & $22 \mathrm{pz}$ \\
\hline 0.79156 & & & & & & & \\
\hline 13.1 & 0.99987 & -0.23596 & $13 \mathrm{~d} 2+0.95553$ & & & & \\
\hline 14.1 & 0.13461 & 0.13661 & $13 \mathrm{~d} 0 \quad 0.84057$ & 2 1s $\quad-0.27455$ & $22 \mathrm{pz}$ & 0.76190 & \\
\hline 1.2 & 2.00000 & -25.06472 & $12 \mathrm{px} \quad 0.99996$ & & & & \\
\hline 2.2 & 2.00000 & -2.63570 & $12 \mathrm{px} \quad 0.99742$ & & & & \\
\hline 3.2 & 2.00000 & -0.62106 & $32 \mathrm{px} \quad 0.91685$ & & & & \\
\hline 4.2 & 1.96567 & -0.64271 & $13 \mathrm{~d} 1+0.31814$ & $22 \mathrm{px} \quad 0.86062$ & & & \\
\hline 5.2 & 1.03081 & -0.25193 & $13 \mathrm{~d} 1+-0.96165$ & 2 2px 0.38913 & & & \\
\hline 1.3 & 2.00000 & -26.29131 & $3 \mathrm{ls} \quad 1.00077$ & & & & \\
\hline 2.3 & 2.00000 & -25.07378 & 12 py 0.99996 & & & & \\
\hline 3.3 & 2.00000 & -2.64717 & 12 py 0.97362 & & & & \\
\hline 4.3 & 2.00000 & -1.53151 & $31 \mathrm{~s} \quad 0.94032$ & & & & \\
\hline 5.3 & 2.00000 & -0.64492 & 12 py -0.34060 & 12 py -0.32502 & 32 py & 0.88490 & \\
\hline 6.3 & 2.00000 & -0.61079 & $32 \mathrm{pz} \quad 0.91190$ & & & & \\
\hline 7.3 & 1.80220 & -0.53527 & $13 \mathrm{~d} 1-0.52314$ & 2 2py $\quad 0.75560$ & & & \\
\hline 8.3 & 0.20060 & 0.02686 & $13 \mathrm{~d} 1-0.83747$ & 2 2py -0.70065 & & & \\
\hline 1.4 & 2.00000 & -0.62761 & $32 \mathrm{px} \quad 0.91784$ & & & & \\
\hline 2.4 & 0.99902 & -0.23790 & $13 d 2-0.99873$ & & & & \\
\hline
\end{tabular}




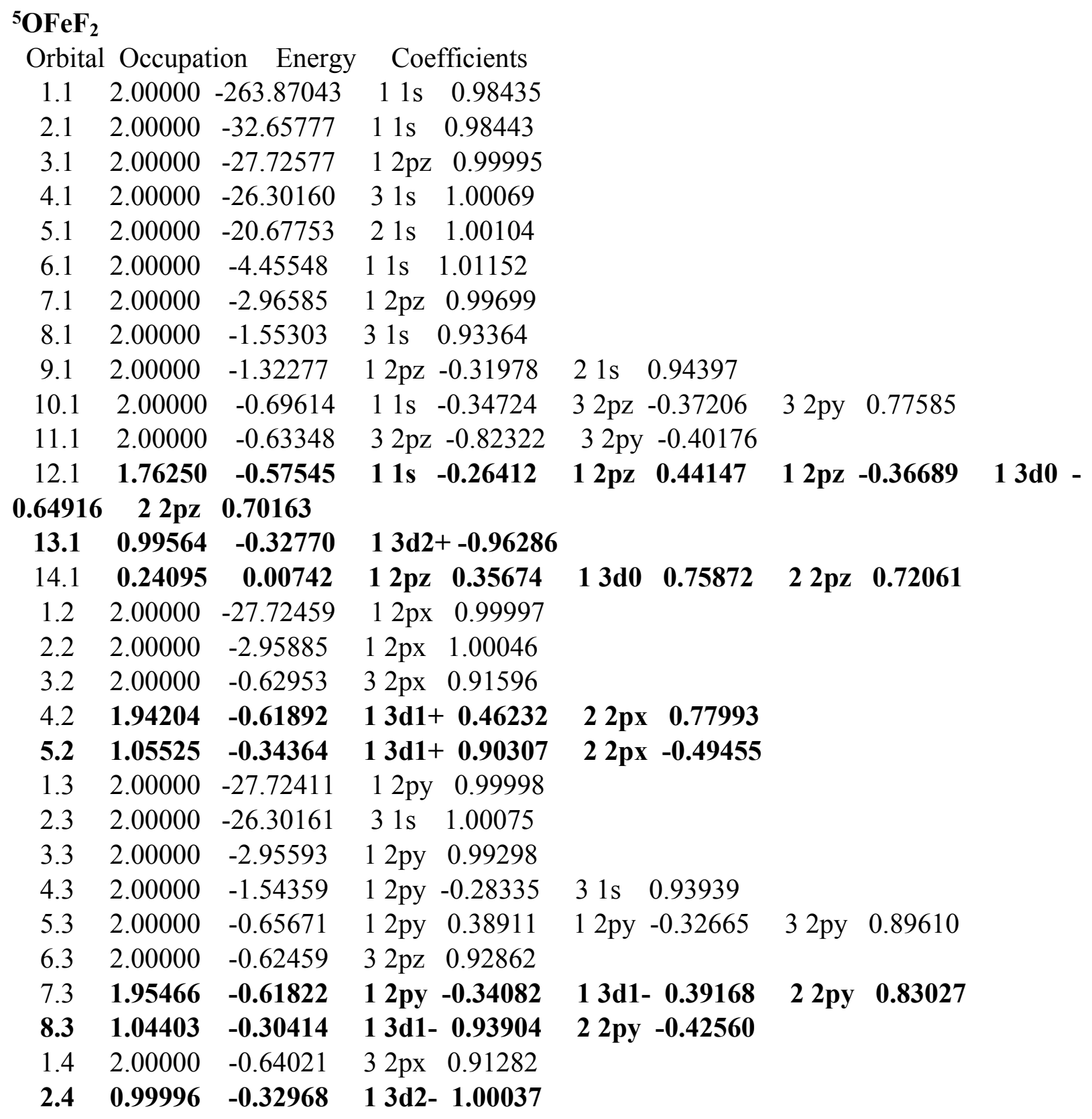




\begin{tabular}{|c|c|c|c|c|c|c|}
\hline \multicolumn{7}{|l|}{${ }^{4} \mathrm{OCoF}_{2}$} \\
\hline Orbital & 1 Occupat & ion Energy & Coefficients & & & \\
\hline 1.1 & 2.00000 & -285.92939 & $11 \mathrm{~s} \quad 0.98322$ & & & \\
\hline 2.1 & 2.00000 & -35.64946 & $11 \mathrm{~s} \quad 0.98309$ & & & \\
\hline 3.1 & 2.00000 & -30.42492 & $12 \mathrm{pz} \quad 0.99991$ & & & \\
\hline 4.1 & 2.00000 & -26.30167 & $31 \mathrm{~s} \quad 1.00070$ & & & \\
\hline 5.1 & 2.00000 & -20.72586 & $21 \mathrm{~s} \quad 1.00103$ & & & \\
\hline 6.1 & 2.00000 & -4.76317 & $11 \mathrm{~s} \quad 1.00345$ & & & \\
\hline 7.1 & 2.00000 & -3.04196 & $12 \mathrm{pz} \quad 0.92081$ & $21 \mathrm{~s} \quad 0.28273$ & & \\
\hline 8.1 & 2.00000 & -1.55270 & $31 \mathrm{~s} \quad 0.92908$ & & & \\
\hline 9.1 & 2.00000 & -0.93498 & $13 \mathrm{~d} 2+-0.51480$ & $21 \mathrm{~s} \quad 0.45008$ & $32 \mathrm{pz} \quad 0.39909$ & 32 py - \\
\hline 0.42400 & & & & & & \\
\hline 10.1 & 2.00000 & -0.69442 & $13 \mathrm{~d} 0 \quad-0.36631$ & $13 \mathrm{~d} 2+0.70820$ & $32 \mathrm{pz} \quad 0.51271$ & \\
\hline 11.1 & 2.00000 & -0.63759 & $11 \mathrm{~s} \quad-0.27211$ & $\begin{array}{lll}32 \mathrm{pz} & 0.51539\end{array}$ & 32 py $\quad 0.70362$ & \\
\hline 12.1 & 1.99767 & -1.35724 & $12 \mathrm{pz} \quad 0.38165$ & $12 \mathrm{pz} \quad-0.25719$ & $21 \mathrm{~s} \quad-0.77624$ & $32 \mathrm{pz}$ \\
\hline 0.34591 & 32 py & -0.26402 & & & & \\
\hline 13.1 & 1.81182 & -0.61028 & $11 \mathrm{~s} \quad 0.25060$ & $12 \mathrm{pz} \quad 0.27454$ & $12 \mathrm{pz} \quad 0.25573$ & $13 \mathrm{~d} 0$ \\
\hline 0.57860 & $22 \mathrm{pz}$ & -0.71802 & & & & \\
\hline 14.1 & 0.19455 & 0.00369 & $13 \mathrm{~d} 0 \quad 0.74926$ & $13 \mathrm{~d} 2+0.29019$ & $22 \mathrm{pz} \quad 0.74353$ & \\
\hline 1.2 & 2.00000 & -30.41085 & $12 \mathrm{px} \quad 0.99997$ & & & \\
\hline 2.2 & 2.00000 & -3.18463 & $12 \mathrm{px} \quad 0.99980$ & & & \\
\hline 3.2 & 2.00000 & -0.62832 & $32 \mathrm{px} \quad-0.91919$ & & & \\
\hline 4.2 & 1.92821 & -0.64671 & $13 \mathrm{~d} 1+0.52290$ & $22 \mathrm{px} \quad 0.76323$ & & \\
\hline 5.2 & 1.06969 & -0.35871 & $13 \mathrm{~d} 1+0.86967$ & $22 p x \quad-0.56714$ & & \\
\hline 1.3 & 2.00000 & -30.41947 & 12 ру 0.99997 & & & \\
\hline 2.3 & 2.00000 & -26.30167 & $31 \mathrm{~s} \quad 1.00076$ & & & \\
\hline 3.3 & 2.00000 & -3.19568 & 12 py 0.99367 & & & \\
\hline 4.3 & 2.00000 & -1.54323 & 3 1s 0.94012 & & & \\
\hline 5.3 & 2.00000 & -0.65783 & 32 ру 0.89892 & & & \\
\hline 6.3 & 2.00000 & -0.62378 & $32 \mathrm{pz} \quad 0.92716$ & & & \\
\hline 7.3 & 1.91815 & -0.65326 & $13 \mathrm{~d} 1--0.58060$ & 2 2py -0.73849 & & \\
\hline 8.3 & 1.07977 & -0.35557 & 1 3d1- 0.83592 & 22 ру -0.61634 & & \\
\hline 1.4 & 2.00000 & -0.63816 & $32 \mathrm{px} \quad 0.91675$ & & & \\
\hline 2.4 & 1.00015 & -0.30959 & $13 \mathrm{~d} 2-1.00505$ & & & \\
\hline
\end{tabular}




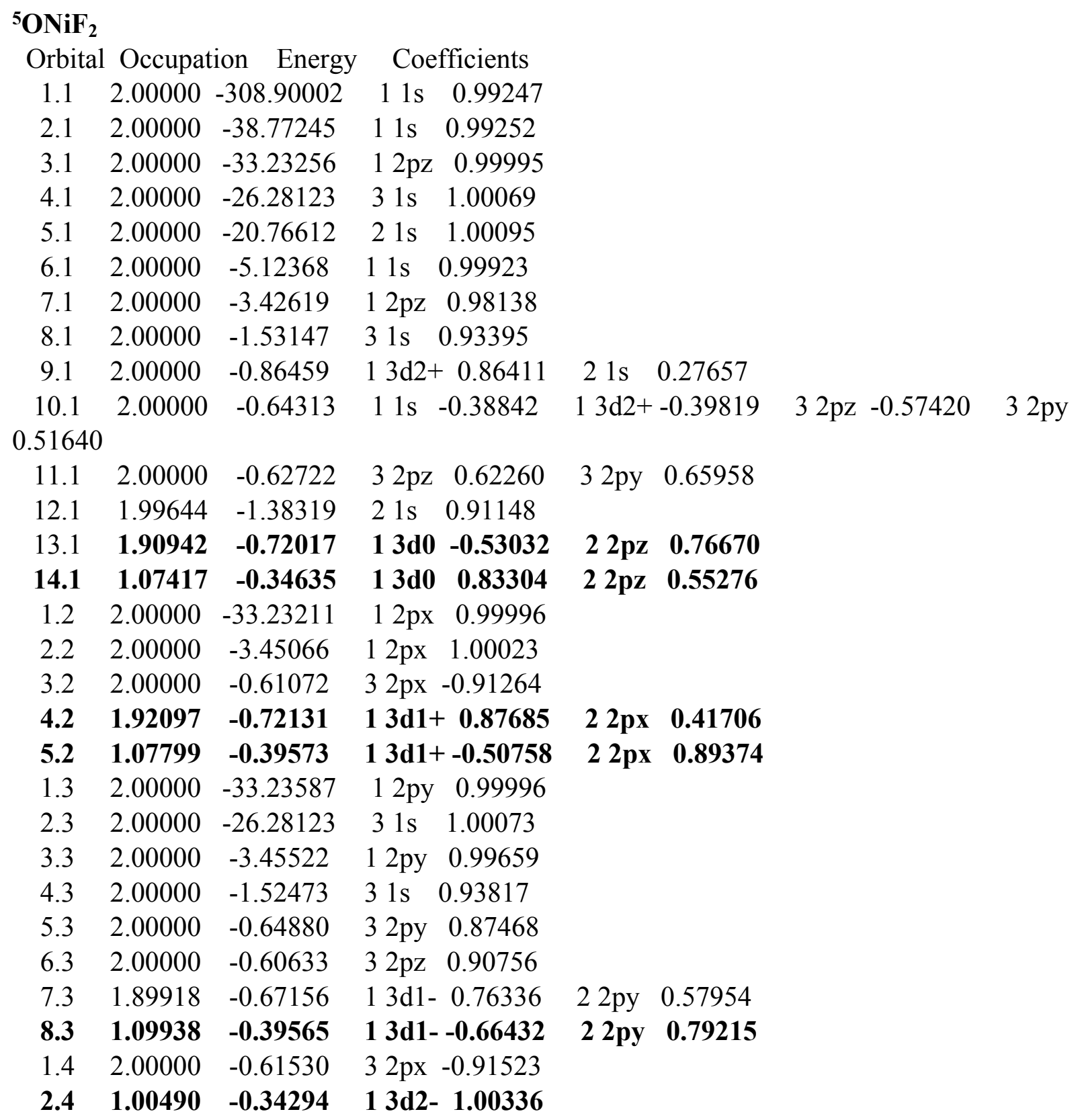




\begin{tabular}{|c|c|c|c|c|c|}
\hline \\
\hline \multicolumn{6}{|c|}{ Orbital Occupation } \\
\hline 1.1 & 2.00000 & -332.74704 & $11 \mathrm{~s} \quad 0.97828$ & & \\
\hline 2.1 & 2.00000 & -41.97256 & $11 \mathrm{~s} \quad 0.97825$ & & \\
\hline 3.1 & 2.00000 & -36.12304 & $12 \mathrm{pz} \quad 0.99997$ & & \\
\hline 4.1 & 2.00000 & -26.24622 & $31 \mathrm{~s} \quad 1.00069$ & & \\
\hline 5.1 & 2.00000 & -20.86330 & $21 \mathrm{~s} \quad 1.00099$ & & \\
\hline 6.1 & 2.00000 & -5.46513 & $11 \mathrm{~s} \quad 1.00203$ & & \\
\hline 7.1 & 2.00000 & -3.66973 & $12 \mathrm{pz} \quad 0.99777$ & & \\
\hline 8.1 & 2.00000 & -1.49007 & $31 \mathrm{~s} \quad 0.92855$ & & \\
\hline 9.1 & 2.00000 & -1.44685 & $21 \mathrm{~s} \quad 0.98056$ & & \\
\hline 10.1 & 2.00000 & -0.77228 & $13 \mathrm{~d} 0-0.43102$ & $13 \mathrm{~d} 2+0.85242$ & \\
\hline 11.1 & 2.00000 & -0.60955 & $11 \mathrm{~s}-0.45016$ & $32 \mathrm{pz}-0.45493$ & 32 py 0.67001 \\
\hline 12.1 & 2.00000 & -0.58539 & $32 \mathrm{pz}-0.73770$ & 32 py -0.52377 & \\
\hline 13.1 & 1.86433 & -0.74874 & $13 \mathrm{~d} 0 \quad-0.32920$ & $22 \mathrm{pz} \quad 0.86833$ & \\
\hline 14.1 & 1.13567 & -0.36130 & $13 \mathrm{~d} 0 \quad 0.83937$ & $13 \mathrm{~d} 2+0.43382$ & $22 \mathrm{pz} \quad 0.42620$ \\
\hline 1.2 & 2.00000 & -36.10676 & $12 \mathrm{px} \quad 0.99997$ & & \\
\hline 2.2 & 2.00000 & -3.65233 & $12 \mathrm{px} \quad 0.99989$ & & \\
\hline 3.2 & 2.00000 & -0.57716 & $32 \mathrm{px} \quad-0.91386$ & & \\
\hline 4.2 & 1.95538 & -0.75471 & $13 \mathrm{~d} 1+0.96920$ & & \\
\hline 5.2 & 1.04462 & -0.43881 & $13 \mathrm{~d} 1+-\mathbf{0 . 2 8 5 7 7}$ & $22 \mathrm{px} \quad 1.00804$ & \\
\hline 1.3 & 2.00000 & -36.11668 & 12 py 0.99997 & & \\
\hline 2.3 & 2.00000 & -26.24622 & $31 \mathrm{~s} \quad 1.00077$ & & \\
\hline 3.3 & 2.00000 & -3.65968 & 12 py 0.99812 & & \\
\hline 4.3 & 2.00000 & -1.48463 & $31 \mathrm{~s} \quad 0.93820$ & & \\
\hline 5.3 & 2.00000 & -0.61069 & 32 py $\quad 0.82779$ & $32 \mathrm{pz}-0.32774$ & \\
\hline 6.3 & 2.00000 & -0.56429 & 32 py -0.37040 & $32 \mathrm{pz}-0.85158$ & \\
\hline 7.3 & 1.89664 & -0.71641 & $13 d 1--0.92901$ & 2 2py -0.31560 & \\
\hline 8.3 & 1.10336 & -0.44806 & $13 d 1--0.38802$ & 2 2py 0.97346 & \\
\hline 1.4 & 2.00000 & -0.78533 & $13 \mathrm{~d} 2-0.98156$ & & \\
\hline 2.4 & 2.00000 & -0.55072 & $13 \mathrm{~d} 2--0.25954$ & $32 \mathrm{px} \quad 0.91901$ & \\
\hline
\end{tabular}


Table S5. OMF CASSCF orbitals. Molecule main axis along z.

\begin{tabular}{|c|c|c|c|c|c|c|c|c|c|}
\hline & ${ }^{1} \mathrm{OScF}$ & ${ }^{2} \mathrm{OTiF}$ & ${ }^{3} \mathrm{OVF}$ & ${ }^{4} \mathrm{OCrF}$ & ${ }^{5} \mathrm{OMnF}$ & ${ }^{6} \mathrm{OFeF}$ & ${ }^{5} \mathrm{OCoF}$ & ${ }^{4} \mathrm{ONiF}$ & ${ }^{3} \mathrm{OCuF}$ \\
\hline Paired $d$ orbs. & & & & & & & 1 & 2 & 3 \\
\hline unpaired $d$ orbs. & 0 & 1 & 2 & 3 & 4 & 5 & 4 & 3 & 2 \\
\hline angle & 125.7 & 136.2 & 149.8 & 137.8 & 126.1 & 180.0 & 180.0 & 180.0 & 180.0 \\
\hline$d(-2) d_{x y}$ & & & 1 & 1 & 1 & 1 & 1 & 2 & 2 \\
\hline$d(-1) d_{y z}$ & & & & & & $1-\mathrm{aO} 2 \mathrm{px}$ & $1-\mathrm{aO} 2 \mathrm{px}$ & $\begin{array}{l}1-\mathrm{aO} 2 \mathrm{px} \\
(1.18)\end{array}$ & $\begin{array}{l}1-\mathrm{aO} 2 \mathrm{px} \\
(1.17)\end{array}$ \\
\hline$d(0) d_{z}^{2}$ & & & & 1 & 1 & 1 & $\begin{array}{l}1+\mathrm{aO} 2 \mathrm{pz} \\
(1.09)\end{array}$ & $\begin{array}{l}1+\mathrm{aO} 2 \mathrm{pz} \\
(1.13)\end{array}$ & $2 \mathrm{~d}_{\mathrm{z}}^{2}+2 \mathrm{pz}$ \\
\hline$d(+1) d_{x z}$ & & & & & 1 & $1-\mathrm{aO} 2 \mathrm{py}$ & $1-\mathrm{aO} 2 \mathrm{py}$ & $\begin{array}{l}1-\mathrm{aO} 2 \mathrm{py} \\
(1.18)\end{array}$ & $\begin{array}{l}1-\mathrm{aO} 2 \mathrm{py} \\
(1.17)\end{array}$ \\
\hline$d(+2) d_{x-y}^{2}{ }^{2}$ & & 1 & 1 & 1 & 1 & 1 & 2 & 2 & 2 \\
\hline
\end{tabular}


Table S6. $\mathrm{OMF}_{2}$ CASSCF orbitals. Molecule $\mathrm{M}-\mathrm{O}$ main axis along $\mathrm{z}$

\begin{tabular}{|c|c|c|c|c|c|c|c|c|c|}
\hline & ${ }^{2} \mathrm{OScF}_{2}$ & ${ }^{1} \mathrm{OTiF}_{2}$ & ${ }^{2} \mathrm{OVF}_{2}$ & ${ }^{3} \mathrm{OCrF}_{2}$ & ${ }^{4} \mathrm{OMnF}_{2}$ & ${ }^{5} \mathrm{OFeF}_{2}$ & ${ }^{4} \mathrm{OCoF}_{2}$ & ${ }^{5} \mathrm{ONiF}_{2}$ & ${ }^{4} \mathrm{OCuF}_{2}$ \\
\hline Paired $d$ orbs. & & & & & & & 1 & 1 & 2 \\
\hline unpaired $d$ orbs. & $1(\mathrm{O})$ & 0 & 1 & 2 & 3 & 4 & 3 & 4 & 3 \\
\hline Angle F-M-F & 118.0 & 114.7 & 116.4 & 119.6 & 115.7 & 108.1 & 106.3 & 113.5 & 112.5 \\
\hline$d(-2) d_{x y}$ & & & & 1 & 1 & 1 & 1 & 1 & \\
\hline$d(-1) d_{y z}$ & & & & & & 1 & 1 & 1.90 & 1.90 \\
\hline$d(0) d_{z}^{2}$ & & & & & & & & 1.07 & 1.14 \\
\hline$d(+1) d_{x z}$ & & & & & 1 & 1 & 1.07 & 1.92 & 1.96 \\
\hline$d(+2) d_{x-y}^{2}{ }^{2}$ & & & 1 & 1 & 1 & 1 & 2 & 2 & \\
\hline
\end{tabular}




\section{DFT NBO Population Analysis.}

On the basis of the DFT NBOs, the Sc-O bonding in ${ }^{1} \mathrm{OScF}$ can be described as having a strong ionic interaction between the $\mathrm{Sc}$ and $\mathrm{O}$ with three highly polar bonds to which $\mathrm{O}$ contributes 80 to $85 \%$ while Sc contributes $15-20 \%$; these can be considered to be backbonding in the ionic model. The oxygen orbitals in the Sc-O bonding possess $90-100 \% p$ character and $d$ character dominates the contributions from Sc to the same bond. The Sc-F bond is even more ionic with $94 \%$ of the contributions coming from the $\mathrm{F}$. The bonding in the open shell species is harder to interpret using the NBO results because of the use of a spin unrestricted calculation. For example, ${ }^{2} \mathrm{OTiF}$ can be described as a molecule containing three Ti-O polar bonds with 75 to $82 \% \mathrm{O}$ and $18-25 \% \mathrm{Ti}$ contributions. The bonds are mostly $p$ character on $\mathrm{O}$ and $d$ character on Ti. The bonding in ${ }^{3} \mathrm{OVF}$ and ${ }^{4} \mathrm{OCrF}$ is essentially the same as that for ${ }^{2} \mathrm{OTiF}$. For ${ }^{5} \mathrm{OMnF}$, the $\mathrm{NBO}$ results show two Mn-O bonds with $\alpha$ electrons and three $\mathrm{Mn}-\mathrm{O}$ bonds with $\beta$ electrons. For $\mathrm{Fe}$ to $\mathrm{Cu}$, there is one $\mathrm{M}-\mathrm{O}$ bond with $\alpha$ electrons and three $\mathrm{M}-\mathrm{O}$ bonds with $\beta$ electrons. The $\alpha$ bonds for $\mathrm{Fe}$ to $\mathrm{Cu}$ have substantially more $\mathrm{O}$ character than $\mathrm{M}$ character as found for the earlier transition metals while the corresponding $\beta$ bonds have more metal character than do the $\mathrm{M}-\mathrm{O}$ bonds of earlier transition metals. As discussed in the manuscript. the results from the DFT/NBO analysis may not adequately describe the number of bonds between metal and oxygen due to the presence of very polar bonds and multireference character.

For ${ }^{1} \mathrm{OTiF}_{2}$, there are two very polar Ti-O bonds with $75 \% \mathrm{O}$ character and $25 \%$ Ti character. These bonds are dominated by $2 p-3 d$ bonding. A third polar Ti-O bond is $87 \%$ O character and $13 \%$ $\mathrm{Ti}$ character. The $\mathrm{O}$ provides predominantly $p$ electrons and the Ti is mostly $66 \% d$ and $30 \% s$. The Ti-F bonds are also very polar with $93 \%$ contributions from F. For trivalent ${ }^{2} \mathrm{OScF}_{2}$, there are two polar Sc-O bonds with $91 \% \mathrm{O}$ character and $9 \% \mathrm{Sc}$ character for the $\alpha$ electrons. One Sc-O bond has $p$ character on $\mathrm{O}$ and $d$ character on $\mathrm{Sc}$, and the other Sc-O bond has $25 \% \mathrm{~s}$ and $75 \% p$ characters on $\mathrm{O}$ and mostly $d$ character on Sc. For the $\beta$ electrons, there is only one polar Sc-O bond with $89 \% \mathrm{O}$ character and $11 \% \mathrm{Sc}$ character forming a $p(\mathrm{O})-d(\mathrm{Sc})$ bond. The bonding in ${ }^{2} \mathrm{OVF}_{2}$ can be described as two polar $\mathrm{V}-\mathrm{O}$ bonds with $72 \% \mathrm{O}$ character and $27 \% \mathrm{~V}$ character. For ${ }^{3} \mathrm{OCrF}_{2}$ there are $2 \mathrm{Cr}-\mathrm{O}$ bonds with $60-80 \% \mathrm{O}$ character and $20-40 \% \mathrm{Cr}$ character depending on if there is $\alpha$ or $\beta$ spin. For ${ }^{4} \mathrm{OMnF}_{2}$ there is only one $\alpha \mathrm{Mn}-\mathrm{O}$ bond with $60 \% \mathrm{O}$ character and there are two $\beta$ bonds with about $70 \% \mathrm{O}$ character. For the ${ }^{5} \mathrm{OFeF}_{2}$ and ${ }^{4} \mathrm{OCoF}_{2}$ there is only one $\alpha \mathrm{Fe}-\mathrm{O}$ bond with $50 \% \mathrm{O}$ character and there are $3 \beta$ bonds with about $55-70 \% \mathrm{O}$ character. For ${ }^{5} \mathrm{ONiF}_{2}$ there is only one $\alpha \mathrm{Ni}-\mathrm{O}$ bond with $95 \% \mathrm{O}$ character and there are two $\beta$ bonds, one with $63 \% \mathrm{O}$ character and one with only $39 \%$ $\mathrm{O}$ character. For ${ }^{4} \mathrm{OCuF}_{2}$ there is only one $\alpha \mathrm{Cu}-\mathrm{O}$ bond with $47 \% \mathrm{O}$ character and there is one $\beta$ bond with only $16 \% \mathrm{O}$ character. The number of $\mathrm{M}-\mathrm{O}$ bonds from a simple valency model would be expected to be 2 for Ti-O. There is not much change in the $\mathrm{M}-\mathrm{O}$ bond distance or the $\mathrm{M}-\mathrm{O}$ frequency from $\mathrm{M}=\mathrm{Ti}$ to $\mathrm{Co}$. The NBOs do predict multiple bond character for the $\mathrm{M}-\mathrm{O}$ bond for these metals, consistent with the frequencies and bond distances. The M-O bond for these species is highly polarized towards oxygen, although as $d$ orbitals become occupied, the amount of metal character increases. For $\mathrm{M}=\mathrm{Ni}$ and $\mathrm{Cu}$, the increase in $\mathrm{M}-\mathrm{O}$ bond distance and decrease in $\mathrm{M}-\mathrm{O}$ frequency are consistent with a decrease in the number of bonding orbitals from the NBO analysis. The excess $\alpha$ spin on the $\mathrm{O}$ atom is $0.7-0.8$ e for $\mathrm{M}=\mathrm{Fe}$ and $\mathrm{Co}$ and 1.5 e for $\mathrm{M}=\mathrm{Ni}$ and $\mathrm{Cu}$, consistent with a decrease on the bond order for $\mathrm{Ni}-\mathrm{O}$ and $\mathrm{Cu}-\mathrm{O}$ bonds. 
Table S7. Population Analysis for OMF at the PW91/aD Level.

\begin{tabular}{|c|c|c|c|c|c|c|c|c|c|}
\hline MOF & $\mathrm{S}^{2} /<\mathrm{S}>^{2}$ & M NPA & Pop M & M Excess Spin & M s $\alpha$ & M d $\alpha$ & Pop O & O 2p $\alpha$ & O 2p $\alpha-\beta$ \\
\hline${ }^{1} \mathrm{OScF}$ & $0.00 / 0.00$ & 1.71 & $4 \mathrm{~s}^{0.03} 3 \mathrm{~d}^{1.26}$ & $3 \mathrm{~d}^{0}$ & 0.01 & 0.63 & $2 \mathrm{p}^{5.02}$ & 2.51 & 0 \\
\hline${ }^{2} \mathrm{OTiF}$ & $0.75 / 0.75$ & 1.33 & $4 \mathrm{~s}^{0.42} 3 \mathrm{~d}^{2.24}$ & $4 \mathrm{~s}^{0.43} \mathrm{~d}^{0.7}$ & 0.40 & 1.46 & $2 \mathrm{p}^{4.78}$ & 2.35 & -0.08 \\
\hline${ }^{3} \mathrm{OVF}$ & $2.02 / 2.00$ & 1.25 & $4 \mathrm{~s}^{0.33} 3 \mathrm{~d}^{3.39}$ & $4 \mathrm{~s}^{0.3} 3 \mathrm{~d}^{1.8}$ & 0.31 & 2.62 & $2 \mathrm{p}^{4.69}$ & 2.26 & -0.17 \\
\hline${ }^{4} \mathrm{OCrF}$ & $3.82 / 3.75$ & 1.21 & $4 \mathrm{~s}^{0.45} 3 \mathrm{~d}^{4.30}$ & $4 \mathrm{~s}^{0.43} \mathrm{~d}^{2.8}$ & 0.42 & 3.54 & $2 \mathrm{p}^{4.65}$ & 2.20 & -0.25 \\
\hline${ }^{5} \mathrm{OMnF}$ & $6.07 / 6.00$ & 1.35 & $4 \mathrm{~s}^{0.39} 3 \mathrm{~d}^{5.22}$ & $4 \mathrm{~s}^{0.33} \mathrm{~d}^{3.5}$ & 0.34 & 4.35 & $2 \mathrm{p}^{4.75}$ & 2.42 & 0.10 \\
\hline${ }^{6} \mathrm{OFeF}$ & $8.76 / 8.75$ & 1.51 & $4 \mathrm{~s}^{0.37} 3 \mathrm{~d}^{6.07}$ & $4 \mathrm{~s}^{0.3} 3 \mathrm{~d}^{3.7}$ & 0.33 & 4.87 & $2 \mathrm{p}^{4.82}$ & 2.84 & 0.87 \\
\hline${ }^{5} \mathrm{OCoF}$ & $6.00 / 6.00$ & 1.41 & $4 \mathrm{~s}^{0.33} 3 \mathrm{~d}^{7.23}$ & $4 \mathrm{~s}^{0.23 \mathrm{~d}^{2.6}}$ & 0.29 & 4.89 & $2 \mathrm{p}^{4.72}$ & 2.88 & 1.04 \\
\hline${ }^{4} \mathrm{ONiF}$ & $3.76 / 3.75$ & 1.26 & $4 \mathrm{~s}^{0.29} 3 \mathrm{~d}^{8.42}$ & $4 \mathrm{~s}^{0.23 \mathrm{~d}^{1.4}}$ & 0.25 & 4.91 & $2 \mathrm{p}^{4.60}$ & 2.90 & 1.20 \\
\hline${ }^{3} \mathrm{OCuF}$ & $2.01 / 2.00$ & 1.15 & $4 \mathrm{~s}^{0.54} 3 \mathrm{~d}^{9.28}$ & $3 \mathrm{~d}^{0.6}$ & 0.26 & 4.93 & $2 \mathrm{p}^{4.51}$ & 2.90 & 1.29 \\
\hline
\end{tabular}

Table S8. Population Analysis for OMF at the B3LYP/aD Level.

\begin{tabular}{|c|c|c|c|c|c|c|c|c|}
\hline OMF & $\mathrm{S}^{2} /<\mathrm{S}>2$ & Pop M & M Excess Spin & Ms $\alpha$ & Md $\alpha$ & Pop O & O $2 p \alpha$ & $\mathrm{O} 2 \mathrm{p} \alpha-\beta$ \\
\hline${ }^{1} \mathrm{OScF}$ & $0.00 / 0.00$ & $4 \mathrm{~s}^{0.03} 3 \mathrm{~d}^{1.16}$ & $3 \mathrm{~d}^{0}$ & 0.01 & 0.58 & $2 \mathrm{p}^{5.09}$ & 2.54 & 0 \\
\hline${ }^{2} \mathrm{OTiF}$ & $0.75 / 0.75$ & $4 \mathrm{~s}^{0.183} \mathrm{~d}^{2.25}$ & $4 \mathrm{~s}^{0.13 \mathrm{~d}^{0.9}}$ & 0.16 & 1.59 & $2 \mathrm{p}^{4.90}$ & 2.41 & 0 \\
\hline${ }^{3} \mathrm{OVF}$ & $2.03 / 2.00$ & $4 \mathrm{~s}^{0.06} 3 \mathrm{~d}^{3.42}$ & $3 \mathrm{~d}^{2.2}$ & 0.05 & 2.80 & $2 \mathrm{p}^{4.82}$ & 2.30 & -0.22 \\
\hline${ }^{4} \mathrm{OCrF}$ & $3.86 / 3.75$ & $4 \mathrm{~s}^{0.40} 3 \mathrm{~d}^{4.21}$ & $4 \mathrm{~s}^{0.3} 3 \mathrm{~d}^{2.9}$ & 0.37 & 3.57 & $2 \mathrm{p}^{4.73}$ & 2.21 & -0.31 \\
\hline${ }^{5} \mathrm{OMnF}$ & $6.67 / 6.00$ & $4 \mathrm{~s}^{0.36} 3 \mathrm{~d}^{5.10}$ & $4 \mathrm{~s}^{0.33} \mathrm{~d}^{4.4}$ & 0.33 & 4.72 & $2 \mathrm{p}^{4.76}$ & 2.02 & -0.72 \\
\hline${ }^{6} \mathrm{OFeF}$ & $8.78 / 8.75$ & $4 \mathrm{~s}^{0.33} 3 \mathrm{~d}^{5.96}$ & $4 \mathrm{~s}^{0.3} 3 \mathrm{~d}^{3.8}$ & 0.29 & 4.89 & $2 \mathrm{p}^{4.90}$ & 2.87 & 0.84 \\
\hline${ }^{5} \mathrm{OCoF}$ & $6.02 / 6.00$ & $4 \mathrm{~s}^{0.29} 3 \mathrm{~d}^{7.15}$ & $4 \mathrm{~s}^{0.23} \mathrm{~d}^{2.7}$ & 0.25 & 4.91 & $2 \mathrm{p}^{4.78}$ & 2.89 & 1.00 \\
\hline${ }^{4} \mathrm{ONiF}$ & $3.77 / 3.75$ & $4 \mathrm{~s}^{0.25} 3 \mathrm{~d}^{8.35}$ & $4 \mathrm{~s}^{0.23} \mathrm{~d}^{1.5}$ & 0.21 & 4.93 & $2 \mathrm{p}^{4.64}$ & 2.91 & 1.21 \\
\hline${ }^{3} \mathrm{OCuF}$ & $2.02 / 2.00$ & $4 \mathrm{~s}^{0.48} 3 \mathrm{~d}^{9.29}$ & $3 \mathrm{~d}^{0.6}$ & 0.22 & 4.93 & $2 \mathrm{p}^{4.48}$ & 2.92 & 1.35 \\
\hline
\end{tabular}


Table S9. Population Analysis for OMF at the BP86/aD Level.

\begin{tabular}{|c|c|c|c|c|c|c|c|c|}
\hline OMF & $\mathrm{S}^{2} /<\mathrm{S}>^{2}$ & Pop M & M Excess Spin & M s $\alpha$ & M d $\alpha$ & Pop O & O $2 p \alpha$ & O $2 p \alpha-\beta$ \\
\hline${ }^{1} \mathrm{OScF}$ & $0.00 / 0.00$ & $4 \mathrm{~s}^{0.03} 3 \mathrm{~d}^{1.26}$ & $3 \mathrm{~d}^{0}$ & 0.01 & 0.63 & $2 \mathrm{p}^{5.03}$ & 2.51 & 0 \\
\hline${ }^{2} \mathrm{OTiF}$ & $0.75 / 0.75$ & $4 \mathrm{~s}^{0.43} 3 \mathrm{~d}^{2.23}$ & $4 \mathrm{~s}^{0.4} 3 \mathrm{~d}^{0.7}$ & 0.40 & 1.45 & $2 \mathrm{p}^{4.78}$ & 2.36 & -0.06 \\
\hline${ }^{3} \mathrm{OVF}$ & $2.02 / 2.00$ & $4 \mathrm{~s}^{0.34} 3 \mathrm{~d}^{3.38}$ & $4 \mathrm{~s}^{0.3} 3 \mathrm{~d}^{1.8}$ & 0.31 & 2.61 & $2 \mathrm{p}^{4.69}$ & 2.26 & -0.17 \\
\hline${ }^{4} \mathrm{OCrF}$ & $3.82 / 3.75$ & $4 \mathrm{~s}^{0.46} 3 \mathrm{~d}^{4.30}$ & $4 \mathrm{~s}^{0.4} 3 \mathrm{~d}^{2.8}$ & 0.43 & 3.54 & $2 \mathrm{p}^{4.65}$ & 2.21 & -0.23 \\
\hline${ }^{5} \mathrm{OMnF}$ & $6.07 / 6.00$ & $4 \mathrm{~s}^{0.39} 3 \mathrm{~d}^{5.22}$ & $4 \mathrm{~s}^{0.3} 3 \mathrm{~d}^{3.5}$ & 0.35 & 4.34 & $2 \mathrm{p}^{4.74}$ & 2.42 & 0.10 \\
\hline${ }^{6} \mathrm{OFeF}$ & $8.76 / 8.75$ & $4 \mathrm{~s}^{0.38} 3 \mathrm{~d}^{6.08}$ & $4 \mathrm{~s}^{0.3} 3 \mathrm{~d}^{3.7}$ & 0.33 & 4.87 & $2 \mathrm{p}^{4.82}$ & 2.84 & 0.87 \\
\hline${ }^{5} \mathrm{OCoF}$ & $6.00 / 6.00$ & $4 \mathrm{~s}^{0.33} 3 \mathrm{~d}^{7.23}$ & $4 \mathrm{~s}^{0.2} 3 \mathrm{~d}^{2.6}$ & 0.29 & 4.89 & $2 \mathrm{p}^{4.72}$ & 2.87 & 1.03 \\
\hline${ }^{4} \mathrm{ONiF}$ & $3.76 / 3.75$ & $4 \mathrm{~s}^{0.29} 3 \mathrm{~d}^{8.42}$ & $4 \mathrm{~s}^{0.2} 3 \mathrm{~d}^{1.4}$ & 0.25 & 4.91 & $2 \mathrm{p}^{4.60}$ & 2.90 & 1.20 \\
\hline${ }^{3} \mathrm{OCuF}$ & $2.01 / 2.00$ & $4 \mathrm{~s}^{0.54} 3 \mathrm{~d}^{9.28}$ & $3 \mathrm{~d}^{0.6}$ & 0.26 & 4.93 & $2 \mathrm{p}^{4.51}$ & 2.89 & 1.27 \\
\hline
\end{tabular}


Table S10. Population Analysis for $\mathrm{OMF}_{2}$ at the PW91/aD Level.

\begin{tabular}{|c|c|c|c|c|c|c|c|c|c|}
\hline $\mathrm{MOF}_{2}$ & $\mathrm{~S}^{2} /<\mathrm{S}>^{2}$ & M NPA & Pop M & M Excess Spin & M s $\alpha$ & M d $\alpha$ & Pop O & O 2p $\alpha$ & O 2p $\alpha-\beta$ \\
\hline${ }^{2} \mathrm{OScF}_{2}$ & $0.75 / 0.75$ & 1.89 & $4 \mathrm{~s}^{0.04} 3 \mathrm{~d}^{1.07}$ & $3 \mathrm{~d}^{0}$ & 0.02 & 0.54 & $2 \mathrm{p}^{4.66}$ & 2.78 & 0.90 \\
\hline${ }^{1} \mathrm{OTiF}_{2}$ & $0.00 / 0.00$ & 1.88 & $4 \mathrm{~s}^{0.05} 3 \mathrm{~d}^{2.06}$ & $3 \mathrm{~d}^{0}$ & 0.03 & 1.03 & $2 \mathrm{p}^{4.74}$ & 2.37 & 0 \\
\hline${ }^{2} \mathrm{OVF}_{2}$ & $0.76 / 0.75$ & 1.59 & $4 \mathrm{~s}^{0.22} 3 \mathrm{~d}^{3.18}$ & $3 \mathrm{~d}^{1}$ & 0.18 & 2.06 & $2 \mathrm{p}^{4.59}$ & 2.25 & -0.09 \\
\hline${ }^{3} \mathrm{OCrF}_{2}$ & $2.04 / 2.00$ & 1.52 & $4 \mathrm{~s}^{0.21} 3 \mathrm{~d}^{4.25}$ & $3 \mathrm{~d}^{2}$ & 0.16 & 3.15 & $2 \mathrm{p}^{4.49}$ & 2.12 & -0.25 \\
\hline${ }^{4} \mathrm{OMnF}_{2}$ & $3.82 / 3.75$ & 1.58 & $4 \mathrm{~s}^{0.20} 3 \mathrm{~d}^{5.19}$ & $3 \mathrm{~d}^{3}$ & 0.14 & 3.98 & $2 \mathrm{p}^{4.55}$ & 2.27 & 0 \\
\hline${ }^{5} \mathrm{OFeF}_{2}$ & $6.03 / 6.00$ & 1.64 & $4 \mathrm{~s}^{0.21} 3 \mathrm{~d}^{6.11}$ & $3 \mathrm{~d}^{3}$ & 0.15 & 4.52 & $2 \mathrm{p}^{4.54}$ & 2.59 & 0.68 \\
\hline${ }^{4} \mathrm{OCoF}_{2}$ & $3.76 / 3.75$ & 1.43 & $4 \mathrm{~s}^{0.33} 3 \mathrm{~d}^{7.20}$ & $3 \mathrm{~d}^{2}$ & 0.16 & 4.52 & $2 \mathrm{p}^{4.41}$ & 2.62 & 0.83 \\
\hline${ }^{5} \mathrm{ONiF}_{2}$ & $6.01 / 6.00$ & 1.51 & $4 \mathrm{~s}^{0.32} 3 \mathrm{~d}^{8.12}$ & $3 \mathrm{~d}^{2}$ & 0.16 & 4.97 & $2 \mathrm{p}^{4.42}$ & 2.95 & 1.48 \\
\hline${ }^{4} \mathrm{OCuF}_{2}$ & $3.76 / 3.75$ & 1.51 & $4 \mathrm{~s}^{0.34} 3 \mathrm{~d}^{9.10}$ & $3 \mathrm{~d}^{1}$ & 0.15 & 4.98 & $2 \mathrm{p}^{4.35}$ & 2.95 & 1.55 \\
\hline
\end{tabular}

Table S11. Population Analysis for $\mathrm{OMF}_{2}$ at the B3LYP/aD Level.

\begin{tabular}{|c|c|c|c|c|c|c|c|c|}
\hline $\mathrm{OMF}_{2}$ & $\mathrm{~S}^{2} /<\mathrm{S}>^{2}$ & Pop M & M Excess Spin & M s $\alpha$ & $\mathrm{Md} \alpha$ & Pop O & O 2p $\alpha$ & $\mathrm{O} 2 \mathrm{p} \alpha-\beta$ \\
\hline${ }^{2} \mathrm{OScF}_{2}$ & $0.75 / 0.75$ & $4 \mathrm{~s}^{0.05} 3 \mathrm{~d}^{0.88}$ & $3 \mathrm{~d}^{0}$ & 0.02 & 0.43 & $2 \mathrm{p}^{4.66}$ & 2.83 & 1.00 \\
\hline${ }^{1} \mathrm{OTiF}_{2}$ & $0.00 / 0.00$ & $4 \mathrm{~s}^{0.06} 3 \mathrm{~d}^{1.93}$ & $3 \mathrm{~d}^{0}$ & 0.03 & 0.87 & $2 \mathrm{p}^{4.79}$ & 2.39 & 0 \\
\hline${ }^{2} \mathrm{OVF}_{2}$ & $0.76 / 0.75$ & $4 \mathrm{~s}^{0.19} 3 \mathrm{~d}^{3.04}$ & $3 \mathrm{~d}^{1}$ & 0.14 & 2.02 & $2 \mathrm{p}^{4.65}$ & 2.27 & -0.11 \\
\hline${ }^{3} \mathrm{OCrF}_{2}$ & $2.06 / 2.00$ & $4 \mathrm{~s}^{0.19} 3 \mathrm{~d}^{4.12}$ & $3 \mathrm{~d}^{2}$ & 0.14 & 3.13 & $2 \mathrm{p}^{4.54}$ & 2.11 & -0.32 \\
\hline${ }^{4} \mathrm{OMnF}_{2}$ & $3.90 / 3.75$ & $4 \mathrm{~s}^{0.18} 3 \mathrm{~d}^{5.06}$ & $3 \mathrm{~d}^{3}$ & 0.13 & 4.03 & $2 \mathrm{p}^{4.58}$ & 2.19 & -0.20 \\
\hline${ }^{5} \mathrm{OFeF}_{2}$ & $6.64 / 6.00$ & $4 \mathrm{~s}^{0.21} 3 \mathrm{~d}^{5.85}$ & $3 \mathrm{~d}^{4}$ & 0.14 & 4.87 & $2 \mathrm{p}^{4.60}$ & 2.08 & -0.44 \\
\hline${ }^{4} \mathrm{OCoF}_{2}$ & $4.41 / 3.75$ & $4 \mathrm{~s}^{0.30} 3 \mathrm{~d}^{6.93}$ & $3 \mathrm{~d}^{3}$ & 0.15 & 4.90 & $2 \mathrm{p}^{4.51}$ & 2.05 & -0.41 \\
\hline${ }^{5} \mathrm{ONiF}_{2}$ & $6.02 / 6.00$ & $4 \mathrm{~s}^{0.30} 3 \mathrm{~d}^{8.01}$ & $3 \mathrm{~d}^{2}$ & 0.14 & 4.97 & $2 \mathrm{p}^{4.38}$ & 2.95 & 1.52 \\
\hline${ }^{4} \mathrm{OCuF}_{2}$ & $3.77 / 3.75$ & $4 \mathrm{~s}^{0.31} 3 \mathrm{~d}^{9.08}$ & $3 \mathrm{~d}^{1}$ & 0.14 & 4.98 & $2 \mathrm{p}^{4.26}$ & 2.96 & 1.66 \\
\hline
\end{tabular}


Table S12. Population Analysis for $\mathrm{OMF}_{2}$ at the BP86/aD Level.

\begin{tabular}{|c|c|c|c|c|c|c|c|c|}
\hline $\mathrm{OMF}_{2}$ & $\mathrm{~S}^{2} /<\mathrm{S}>$ & Pop M & M Excess Spin & M s $\alpha$ & Md $\alpha$ & Pop O & O $2 \mathrm{p} \alpha$ & O $2 \mathrm{p} \alpha-\beta$ \\
\hline${ }^{2} \mathrm{OScF}_{2}$ & $0.75 / 0.75$ & $4 \mathrm{~s}^{0.04} 3 \mathrm{~d}^{1.07}$ & $3 \mathrm{~d}^{0}$ & 0.02 & 0.54 & $2 \mathrm{p}^{4.66}$ & 2.85 & 1.00 \\
\hline${ }^{1} \mathrm{OTiF}_{2}$ & $0.00 / 0.00$ & $4 \mathrm{~s}^{0.06} 3 \mathrm{~d}^{2.06}$ & $3 \mathrm{~d}^{0}$ & 0.03 & 1.03 & $2 \mathrm{p}^{4.74}$ & 2.37 & 0 \\
\hline${ }^{2} \mathrm{OVF}_{2}$ & $0.76 / 0.75$ & $4 \mathrm{~s}^{0.22} 3 \mathrm{~d}^{3.18}$ & $3 \mathrm{~d}^{1}$ & 0.18 & 2.06 & $2 \mathrm{p}^{4.59}$ & 2.26 & -0.08 \\
\hline${ }^{3} \mathrm{OCrF}_{2}$ & $2.04 / 2.00$ & $4 \mathrm{~s}^{0.21} 3 \mathrm{~d}^{4.25}$ & $3 \mathrm{~d}^{2}$ & 0.16 & 3.14 & $2 \mathrm{p}^{4.50}$ & 2.13 & -0.24 \\
\hline${ }^{4} \mathrm{OMnF}_{2}$ & $3.82 / 3.75$ & $4 \mathrm{~s}^{0.20} 3 \mathrm{~d}^{5.20}$ & $3 \mathrm{~d}^{3}$ & 0.15 & 3.97 & $2 \mathrm{p}^{4.54}$ & 2.28 & 0 \\
\hline${ }^{5} \mathrm{OFeF}_{2}$ & $6.03 / 6.00$ & $4 \mathrm{~s}^{0.22} 3 \mathrm{~d}^{6.12}$ & $3 \mathrm{~d}^{3}$ & 0.15 & 4.51 & $2 \mathrm{p}^{4.53}$ & 2.60 & 0.67 \\
\hline${ }^{4} \mathrm{OCoF}_{2}$ & $3.76 / 3.75$ & $4 \mathrm{~s}^{0.33} 3 \mathrm{~d}^{7.21}$ & $3 \mathrm{~d}^{2}$ & 0.16 & 4.52 & $2 \mathrm{p}^{4.41}$ & 2.62 & 0.83 \\
\hline${ }^{5} \mathrm{ONiF}_{2}$ & $6.01 / 6.00$ & $4 \mathrm{~s}^{0.33} 3 \mathrm{~d}^{8.12}$ & $3 \mathrm{~d}^{2}$ & 0.16 & 4.97 & $2 \mathrm{p}^{4.42}$ & 2.94 & 1.46 \\
\hline${ }^{4} \mathrm{OCuF}_{2}$ & $3.76 / 3.75$ & $4 \mathrm{~s}^{0.34} 3 \mathrm{~d}^{9.10}$ & $3 \mathrm{~d}^{1}$ & 0.16 & 4.98 & $2 \mathrm{p}^{4.35}$ & 2.94 & 1.53 \\
\hline
\end{tabular}


Table S13. Total Atomization Energies for OMF.

\begin{tabular}{|c|c|c|c|c|c|c|c|c|c|}
\hline Molecule & $\Delta \mathrm{E}_{\mathrm{ZPE}}$ & $\Delta \mathrm{E}_{\mathrm{aD}(\mathrm{V})}$ & $\Delta \mathrm{E}_{\mathrm{aT}(\mathrm{V})}$ & $\Delta \mathrm{E}_{\mathrm{aQ}(\mathrm{V})}$ & $\begin{array}{c}\Delta \mathrm{E}_{\mathrm{CBS}} \\
(\mathrm{V})\end{array}$ & $\begin{array}{c}\Delta \mathrm{E}_{\mathrm{aWD}} \\
(\mathrm{CV})\end{array}$ & $\begin{array}{c}\Delta \mathrm{E}_{\mathrm{aWT}} \\
(\mathrm{CV})\end{array}$ & $\begin{array}{c}\Delta \mathrm{E}_{\mathrm{aWQ}} \\
(\mathrm{CV})\end{array}$ & $\begin{array}{c}\Delta \mathrm{E}_{\mathrm{CBS}} \\
(\mathrm{CV})\end{array}$ \\
\hline${ }^{1} \mathrm{OScF}$ & -2.55 & 281.36 & 289.27 & 292.91 & $\mathbf{2 9 5 . 0 9}$ & 284.12 & 293.31 & 297.67 & $\mathbf{3 0 0 . 2 9}$ \\
\hline${ }^{2} \mathrm{OTiF}$ & -2.57 & 279.54 & 286.94 & 291.18 & $\mathbf{2 9 3 . 7 8}$ & 281.35 & 289.33 & 293.75 & $\mathbf{2 9 6 . 4 7}$ \\
\hline${ }^{3} \mathrm{OVF}$ & -2.56 & 257.91 & 265.80 & 269.78 & $\mathbf{2 7 2 . 2 0}$ & 260.87 & 268.66 & 272.48 & $\mathbf{2 7 4 . 7 9}$ \\
\hline${ }^{4} \mathrm{OCrF}$ & -2.60 & 228.76 & 236.45 & 240.26 & $\mathbf{2 4 2 . 5 7}$ & 231.19 & 238.33 & 241.45 & $\mathbf{2 4 3 . 3 2}$ \\
\hline${ }^{5} \mathrm{OMnF}$ & -1.89 & 186.84 & 194.62 & 198.23 & $\mathbf{2 0 2 . 5 1}$ & 187.37 & 194.64 & 198.24 & $\mathbf{2 0 0 . 4 1}$ \\
\hline${ }^{6} \mathrm{OFeF}$ & -2.26 & 206.89 & 213.91 & 217.20 & $\mathbf{2 1 9 . 1 9}$ & 206.40 & 212.79 & 216.26 & $\mathbf{2 1 8 . 3 9}$ \\
\hline${ }^{5} \mathrm{OCoF}$ & -2.59 & 200.79 & 208.37 & 211.77 & $\mathbf{2 1 3 . 7 8}$ & 200.69 & 207.87 & 211.35 & $\mathbf{2 1 3 . 4 5}$ \\
\hline${ }^{4} \mathrm{ONiF}$ & -2.68 & 192.70 & 200.18 & 203.37 & $\mathbf{2 0 5 . 2 7}$ & 187.17 & 195.98 & 199.67 & $\mathbf{2 0 1 . 8 7}$ \\
\hline${ }^{3} \mathrm{OCuF}$ & -2.30 & 157.65 & 163.46 & 165.62 & $\mathbf{1 6 7 . 0 3}$ & 155.13 & 160.11 & 161.99 & $\mathbf{1 6 3 . 0 8}$ \\
\hline
\end{tabular}

\begin{tabular}{|c|c|c|c|c|c|c|c|c|c|}
\hline & & \multicolumn{4}{|c|}{ HF Orbital } & \multicolumn{4}{|c|}{ PW91 Orbital } \\
\hline Molecule & $\Delta \mathrm{E}_{\mathrm{ZPE}}$ & $\begin{array}{c}\Delta \mathrm{E}_{\mathrm{aWD}-} \\
\mathrm{DK}(\mathrm{CV})\end{array}$ & $\begin{array}{c}\Delta \mathrm{E}_{\mathrm{aWT}-} \\
\mathrm{DK}(\mathrm{CV})\end{array}$ & $\begin{array}{c}\Delta \mathrm{E}_{\mathrm{aWQ}}- \\
\mathrm{DK}(\mathrm{CV})\end{array}$ & $\begin{array}{c}\Delta \mathbf{E}_{\text {CBS- }} \\
\text { DK(CV) }\end{array}$ & $\begin{array}{l}\Delta \mathrm{E}_{\mathrm{aWD}} \\
-\mathrm{DK}(\mathrm{CV})\end{array}$ & $\begin{array}{c}\Delta \mathrm{E}_{\mathrm{aWT}}- \\
\mathrm{DK}(\mathrm{CV})\end{array}$ & $\begin{array}{l}\Delta \mathrm{E}_{\mathrm{aWQ}} \\
-\mathrm{DK}(\mathrm{CV})\end{array}$ & $\begin{array}{l}\Delta \mathbf{E}_{\mathrm{CBS}}- \\
\mathrm{DK}(\mathrm{CV})\end{array}$ \\
\hline${ }^{1} \mathrm{OScF}$ & -2.55 & 284.43 & 292.87 & 297.25 & 299.92 & 284.35 & 292.61 & 296.97 & 299.63 \\
\hline${ }^{2} \mathrm{OTiF}$ & -2.57 & 280.01 & 288.75 & 293.15 & 295.83 & 282.55 & 291.15 & 295.50 & 298.13 \\
\hline${ }^{3} \mathrm{OVF}$ & -2.56 & 259 & 266.86 & & 27 & 263.26 & 270.87 & 274.62 & 276.89 \\
\hline${ }^{4} \mathrm{OCrF}$ & -2.60 & 229 & 235.69 & 0 & 240 & 233.11 & 239.29 & 241.98 & 243.58 \\
\hline${ }^{5} \mathrm{OMnF}$ & -1.89 & 187. & 193.12 & 196 & 199.40 & 188.44 & 194.88 & 198.31 & 200.41 \\
\hline${ }^{6} \mathrm{OFeF}$ & -2.26 & 206.42 & 211.58 & 215.12 & 217.33 & 205.42 & 210.63 & 213.97 & 216.05 \\
\hline${ }^{5} \mathrm{OCoF}$ & -2.59 & 200.17 & 205.78 & 209.34 & 211.56 & 198.58 & 203.92 & 207.46 & 209.67 \\
\hline${ }^{4} \mathrm{ONiF}$ & -2.68 & 187.30 & 193.91 & 197.68 & 199.99 & 187.05 & 193.47 & 197.25 & 199.58 \\
\hline${ }^{3} \mathrm{OCuF}$ & -2.30 & 155.70 & 158.40 & 160.23 & 161.38 & 155.13 & 157.70 & 159.41 & 160.48 \\
\hline \multicolumn{10}{|c|}{ Brueckner } \\
\hline${ }^{1} \mathrm{OScF}$ & -2.55 & 283.58 & 292.03 & 296.45 & 299.15 & & & & \\
\hline
\end{tabular}


Table S14. Total Atomization Energies for $\mathrm{OMF}_{2}$

\begin{tabular}{|c|c|c|c|c|c|c|c|c|c|}
\hline Molecule & $\Delta \mathrm{E}_{\mathrm{ZPE}}$ & $\Delta \mathrm{E}_{\mathrm{aD}(\mathrm{V})}$ & $\Delta \mathrm{E}_{\mathrm{aT}(\mathrm{V})}$ & $\Delta \mathrm{E}_{\mathrm{aQ}(\mathrm{V})}$ & $\begin{array}{c}\Delta \mathbf{E}_{\mathbf{C B S}} \\
(\mathrm{V})\end{array}$ & $\begin{array}{c}\Delta \mathrm{E}_{\mathrm{aWD}} \\
(\mathrm{CV})\end{array}$ & $\begin{array}{c}\Delta \mathrm{E}_{\mathrm{aWT}} \\
(\mathrm{CV})\end{array}$ & $\begin{array}{c}\Delta \mathrm{E}_{\mathrm{aWQ}} \\
(\mathrm{CV})\end{array}$ & $\begin{array}{c}\Delta \mathbf{E}_{\mathbf{C B S}} \\
(\mathbf{C V})\end{array}$ \\
\hline${ }^{2} \mathrm{OScF}_{2}$ & -3.55 & 372.06 & 378.97 & 382.55 & $\mathbf{3 8 4 . 7 2}$ & 375.18 & 384.30 & 388.57 & $\mathbf{3 9 1 . 1 5}$ \\
\hline${ }^{1} \mathrm{OTiF}_{2}$ & -4.28 & 426.46 & 435.88 & 441.25 & $\mathbf{4 4 4 . 5 6}$ & 430.46 & 439.56 & 444.67 & $\mathbf{4 4 7 . 8 2}$ \\
\hline${ }^{2} \mathrm{OVF}_{2}$ & -4.41 & 397.77 & 408.46 & 413.67 & $\mathbf{4 1 6 . 8 1}$ & 401.72 & 411.91 & 416.85 & $\mathbf{4 1 9 . 8 4}$ \\
\hline${ }^{3} \mathrm{OCrF}_{2}$ & -4.46 & 338.95 & 350.89 & 355.96 & $\mathbf{3 5 8 . 9 8}$ & 343.21 & 352.94 & 357.19 & $\mathbf{3 5 9 . 7 4}$ \\
\hline${ }^{4} \mathrm{OMnF}_{2}$ & -4.30 & 278.26 & 291.27 & 296.74 & $\mathbf{3 0 2 . 0 9}$ & 282.70 & 294.48 & 299.62 & $\mathbf{3 0 2 . 6 9}$ \\
\hline${ }^{5} \mathrm{OFeF}_{2}$ & -3.59 & 281.71 & 292.41 & 296.91 & $\mathbf{2 9 9 . 5 7}$ & 282.17 & 291.51 & 296.16 & $\mathbf{2 9 8 . 9 7}$ \\
\hline${ }^{4} \mathrm{OCoF}_{2}$ & -3.63 & 257.08 & 268.40 & 272.79 & $\mathbf{2 7 5 . 3 5}$ & 258.92 & 268.83 & 273.24 & $\mathbf{2 7 5 . 8 7}$ \\
\hline${ }^{5} \mathrm{ONiF}_{2}$ & -3.58 & 235.70 & 244.58 & 248.18 & $\mathbf{2 5 0 . 3 1}$ & 234.33 & 241.51 & 245.18 & $\mathbf{2 4 7 . 4 2}$ \\
\hline${ }^{4} \mathrm{OCuF}_{2}$ & -3.33 & 183.75 & 191.25 & 194.13 & $\mathbf{1 9 5 . 9 7}$ & 182.91 & 188.96 & 191.37 & $\mathbf{1 9 2 . 7 8}$ \\
\hline
\end{tabular}

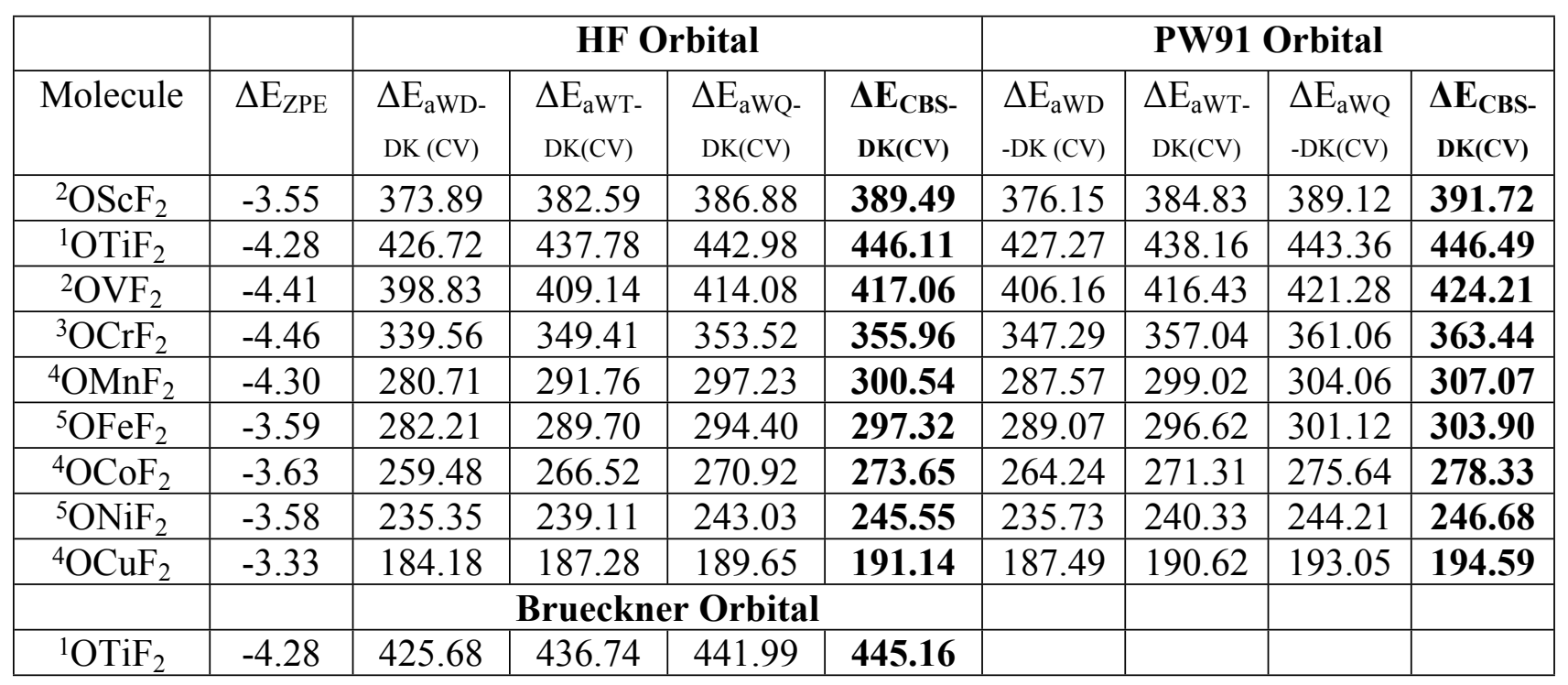


Table S15. T1 values for OMF

\begin{tabular}{|c|c|c|c|c|}
\hline Molecule & $T_{1}$ (PP/aT-HF) & $T_{1}$ (DK/awT-HF) & $T_{1}$ (DK/awT-PW91) & $\begin{array}{c}T_{1} \text { (DK/awT- } \\
\text { Brueckner })\end{array}$ \\
\hline${ }^{1} \mathrm{OScF}$ & 0.02778 & 0.02562 & 0.02011 & 0.00593 \\
\hline${ }^{2} \mathrm{OTiF}$ & 0.05529 & 0.03464 & 0.05343 & \\
\hline${ }^{3} \mathrm{OVF}$ & 0.05225 & 0.04171 & 0.02320 & \\
\hline${ }^{4} \mathrm{OCrF}$ & 0.05959 & 0.04976 & 0.02454 & \\
\hline${ }^{5} \mathrm{OMnF}$ & 0.06277 & 0.05221 & 0.03884 & \\
\hline${ }^{6} \mathrm{OFeF}$ & 0.07353 & 0.06464 & 0.02434 & \\
\hline${ }^{5} \mathrm{OCoF}$ & 0.09005 & 0.06888 & 0.02420 & \\
\hline${ }^{4} \mathrm{ONiF}$ & 0.13363 & 0.06098 & 0.02515 & \\
${ }^{3} \mathrm{OCuF}$ & 0.09316 & 0.07069 & 0.02559 & \\
\hline
\end{tabular}

Table S16. T1 values for $\mathrm{OMF}_{2}$

\begin{tabular}{|c|c|c|c|c|}
\hline Molecule & $T_{1}$ (PP/aT-HF) & $T_{1}$ (DK/awT-HF) & $T_{1}$ (DK/awT-PW91) & $\begin{array}{c}T_{1} \text { (DK/awT- } \\
\text { Brueckner) }\end{array}$ \\
\hline${ }^{2} \mathrm{OScF}_{2}$ & 0.01956 & 0.01867 & 0.02865 & \\
\hline${ }^{1} \mathrm{OTiF}_{2}$ & 0.03464 & 0.02680 & 0.01951 & 0.00524 \\
\hline${ }^{2} \mathrm{OVF}_{2}$ & 0.03761 & 0.03320 & 0.02355 & \\
\hline${ }^{3} \mathrm{OCrF}_{2}$ & 0.04139 & 0.03595 & 0.02384 & \\
\hline${ }^{4} \mathrm{OMnF}_{2}$ & 0.04925 & 0.04219 & 0.02697 & \\
\hline${ }^{5} \mathrm{OFeF}_{2}$ & 0.04431 & 0.03807 & 0.02775 & \\
\hline${ }^{4} \mathrm{OCoF}_{2}$ & 0.03123 & 0.03647 & 0.02679 & \\
\hline${ }^{5} \mathrm{ONiF}_{2}$ & 0.08322 & 0.04926 & 0.03540 & \\
\hline${ }^{4} \mathrm{OCuF}_{2}$ & 0.07739 & 0.06458 & 0.03806 & \\
\hline
\end{tabular}

Table S17. Experimental Atomic Ionization Potentials, Hardness and Electronegativity for the +III oxidation state in $\mathrm{eV}$

\begin{tabular}{|l|l|l|l|l|r|r|}
\hline Metal & IP1 & IP2 & IP3 & IP4 & $\eta$ & $\sum($ IP(1-3) \\
\hline $\mathrm{Sc}$ & 6.56 & 12.80 & 24.76 & 73.49 & 24.37 & 44.12 \\
\hline $\mathrm{Ti}$ & 6.83 & 13.58 & 27.49 & 43.27 & 7.89 & 47.9 \\
\hline $\mathrm{V}$ & 6.75 & 14.63 & 29.31 & 46.71 & 8.70 & 50.69 \\
\hline $\mathrm{Cr}$ & 6.77 & 16.49 & 30.96 & 49.16 & 9.10 & 54.22 \\
\hline $\mathrm{Mn}$ & 7.43 & 15.64 & 33.67 & 51.21 & 8.77 & 56.74 \\
\hline $\mathrm{Fe}$ & 7.90 & 16.20 & 30.65 & 54.91 & 12.13 & 54.75 \\
\hline $\mathrm{Co}$ & 7.88 & 17.08 & 33.50 & 51.27 & 8.89 & 58.46 \\
\hline $\mathrm{Ni}$ & 7.64 & 18.17 & 35.19 & 54.92 & 9.87 & 61 \\
\hline $\mathrm{Cu}$ & 7.73 & 20.29 & 36.84 & 57.38 & 10.27 & 64.86 \\
\hline
\end{tabular}


Table S18. Second order perturbation theory energy analysis from NBOs analysis for OMF and $\mathrm{OMF}_{2}$.

\section{OScF}

7. (1.98959) LP (1) O $1 \quad$ s( 89.27\%)p 0.12( $10.73 \%)$ d $0.00(0.00 \%)$

8. (1.99608) LP ( 1) F $3 \quad$ s( 81.32\%)p 0.23(18.68\%)d 0.00( $0.00 \%)$

9. (1.94351) LP (2) F $3 \quad$ s( $1.84 \%)$ p53.41( $98.10 \%) d 0.03(0.06 \%)$

10. (1.91650) LP (3) F $3 \quad$ s( $0.00 \%)$ p $1.00(99.92 \%)$ d $0.00(0.08 \%)$

11. (1.99974) BD ( 1) O 1-Sc 2

(83.74\%) $\quad 0.9151 * \mathrm{O} 1 \mathrm{~s}(0.00 \%) \mathrm{p} 1.00(99.74 \%) \mathrm{d} 0.00(0.26 \%)$

( $16.26 \%) \quad 0.4033 *$ Sc 2 s( $0.00 \%)$ p 1.00( 0.66\%)d99.99( 99.25\%) f $0.14(0.09 \%)$

12. (1.99590) BD (2) O 1-Sc 2

( $80.79 \%) \quad 0.8988^{*} \mathrm{O} 1 \mathrm{~s}(10.62 \%) \mathrm{p} 8.38(89.07 \%) \mathrm{d} 0.03(0.31 \%)$

(19.21\%) $0.4383 *$ Sc 2 s( $2.48 \%)$ p 0.32( $0.80 \%) d 38.87(96.45 \%)$ f $0.11(0.27 \%)$

13. (1.98004) BD (3) O 1-Sc 2

( 85.49\%) $0.9246^{*}$ O $1 \mathrm{~s}(0.24 \%) p 99.99(99.54 \%) d 0.93(0.22 \%)$

(14.51\%) $0.3810 * \operatorname{Sc} 2 \mathrm{~s}(2.05 \%) \mathrm{p} 0.60(1.22 \%) \mathrm{d} 47.15(96.54 \%)$ f 0.09 ( $0.19 \%)$

14. (1.98347) BD ( 1)Sc 2- F 3

( 6.08\%) $0.2466 * \operatorname{Sc} 2 \mathrm{~s}(14.27 \%) \mathrm{p} 0.03(0.41 \%) \mathrm{d} 5.96(85.06 \%)$ f $0.02(0.26 \%)$

(93.92\%) $0.9691 *$ F 3 s( 16.84\%)p 4.93( 83.03\%)d 0.01( 0.13\%)

\section{${ }^{2} \mathrm{OTiF}$}

Alpha

7. (0.99379) LP ( 1) O $1 \quad$ s( 87.89\%)p 0.14( $12.10 \%) d 0.00(0.00 \%)$

8. (0.99781) LP ( 1)Ti $2 \quad \mathrm{~s}(33.98 \%)$ p 0.03( $1.09 \%) \mathrm{d} 1.91(64.84 \%)$ f $0.00(0.09 \%)$

9. (0.99799) LP ( 1) F $3 \quad$ s( 80.64\%)p 0.24( $19.36 \%) d 0.00(0.00 \%)$

10. (0.95957) LP (2) F $3 \quad$ s( $1.44 \%)$ p $68.51(98.49 \%) d 0.05(0.08 \%)$

11. (0.93611) LP (3) F $3 \quad$ s( $0.00 \%)$ p $1.00(99.91 \%)$ d $0.00(0.09 \%)$

12. (0.90157) LP (4) F 3 s( $17.91 \%)$ p 4.57( 81.94\%)d 0.01( $0.15 \%)$

13. (0.99909) BD ( 1) O 1-Ti 2

( 74.62\%) $0.8638^{*} \mathrm{O} 1 \mathrm{~s}(12.20 \%) \mathrm{p} 7.17(87.56 \%) \mathrm{d} 0.02(0.24 \%)$

(25.38\%) $0.5038^{*} \mathrm{Ti} 2 \mathrm{~s}(10.02 \%) \mathrm{p} 0.11(1.06 \%) \mathrm{d} 8.85(88.65 \%)$ f $0.03(0.27 \%)$

14. (0.99986) BD (2) O 1-Ti 2

( 77.98\%) $0.8830 *$ O $1 \mathrm{~s}(0.00 \%) \mathrm{p} 1.00(99.76 \%) \mathrm{d} 0.00(0.24 \%)$

( $22.02 \%) \quad 0.4693 * \mathrm{Ti} 2 \mathrm{~s}(0.00 \%) \mathrm{p} 1.00(0.54 \%) \mathrm{d} 99.99$ (99.31\%) f $0.29(0.16 \%)$

15. (0.99849) BD (3) O 1-Ti 2

(78.90\%) $0.8882 *$ O $1 \mathrm{~s}(0.05 \%) p 99.99(99.73 \%) \mathrm{d} 4.32(0.22 \%)$

(21.10\%) $0.4594 * \operatorname{Ti} 2 \mathrm{~s}(0.03 \%) \mathrm{p} 19.89(0.64 \%) \mathrm{d} 99.99(99.16 \%)$ f $5.44(0.17 \%)$ 


\section{Beta}

7. (0.99053) LP ( 1$) \mathrm{O} 1$

s( $88.10 \%)$ p 0.14( $11.90 \%)$ d $0.00(0.00 \%)$

8. (0.99693) LP ( 1) F 3

s( 80.89\%)p 0.24( $19.11 \%) d 0.00(0.00 \%)$

9. (0.96168) LP ( 2) F 3

s( $1.41 \%)$ p69.65( $98.51 \%) d 0.05(0.08 \%)$

10. (0.94317) LP ( 3) F 3 s( $0.00 \%)$ p 1.00( 99.91\%)d 0.00( 0.09\%)

11. (0.90416) LP ( 4) F $3 \quad$ s( $17.68 \%)$ p 4.65( 82.17\%)d 0.01( $0.15 \%)$

12. $(0.99953) \mathrm{BD}$ ( 1) O 1-Ti 2

$\left(\begin{array}{ll}75.80 \%) & 0.8706 * \text { O } 1 \mathrm{~s}(11.99 \%) p ~ 7.32(87.77 \%) \mathrm{d} 0.02(0.24 \%)\end{array}\right.$

(24.20\%) $0.4920 *$ Ti 2 s( 2.94\%)p 0.21( 0.62\%)d32.77( 96.18\%) f $0.09(0.27 \%)$

13. (0.99990) BD ( 2) O 1-Ti 2

$\begin{array}{ll}(81.05 \%) & 0.9003 * \text { O } 1 \mathrm{~s}(0.00 \%) p ~ 1.00(99.77 \%) \mathrm{d} 0.00(0.23 \%)\end{array}$

( $18.95 \%) \quad 0.4353 * \mathrm{Ti} 2 \mathrm{~s}(0.00 \%) \mathrm{p} 1.00(0.36 \%) \mathrm{d} 99.99(99.46 \%)$ f $0.50(0.18 \%)$

14. (0.99900) BD ( 3) O 1-Ti 2

( 81.96\%) $0.9053 *$ O $1 \mathrm{~s}(0.01 \%) p 99.99(99.78 \%) \mathrm{d} 16.87(0.21 \%)$

( $18.04 \%) \quad 0.4247 *$ Ti 2 s( 0.04\%)p15.09( 0.60\%)d99.99(99.16\%) f $5.10(0.20 \%)$

\section{${ }^{3}$ OVF \\ Alpha}

9. (0.99349) LP (2) V $2 \quad s(0.00 \%) p 1.00(1.02 \%) \mathrm{d} 96.82(98.95 \%)$

10. (0.99813) LP ( 1) F 3 f $0.03(0.03 \%)$

11. (0.95953) LP ( 2) F 3

s( 82.14\%)p 0.22( $17.86 \%) d 0.00(0.00 \%)$

12. $(0.95115)$ LP ( 3$)$ F 3

s( $0.00 \%)$ p $1.00(99.91 \%) d 0.00(0.09 \%)$

13. (0.89570) LP ( 4) F 3

s( 2.34\%)p41.71( 97.57\%)d 0.04( $0.09 \%)$

14. (0.99944) BD ( 1) O 1- V 2

s( $15.51 \%)$ p 5.44( 84.32\%)d 0.01( 0.16\%)

( 70.93\%) $0.8422 *$ O $1 \mathrm{~s}(10.25 \%)$ p 8.73( 89.49\%)d 0.03( $0.27 \%)$

(29.07\%) $0.5392 * \mathrm{~V} 2 \mathrm{~s}(9.55 \%)$ p 0.08( $0.79 \%) \mathrm{d} 9.37(89.44 \%)$ f $0.02(0.22 \%)$

15. (0.99886) BD ( 2) O 1- V 2

( 74.69\%) $0.8642 *$ O 1 s( $0.22 \%) p 99.99(99.52 \%) d 1.13(0.25 \%)$

(25.31\%) $0.5031 * \mathrm{~V} 2 \mathrm{~s}(0.35 \%) \mathrm{p} 2.04(0.71 \%) \mathrm{d} 99.99(98.85 \%)$ f $0.28(0.10 \%)$

16. (0.99905) BD ( 3) O 1- V 2

$(74.73 \%) \quad 0.8645 *$ O 1 s( $0.00 \%)$ p 1.00 (99.72\%)d $0.00(0.28 \%)$

(25.27\%) $0.5027 *$ V 2 s( $0.00 \%) p$ 1.00( 1.25\%)d79.11(98.67\%) f $0.06(0.08 \%)$

\section{Beta}

7. (0.99093) LP ( 1) O 1

s( 87.65\%)p 0.14( $12.35 \%) d 0.00(0.00 \%)$

8. (0.99758) LP ( 1) F 3

9. (0.95693) LP ( 2) F 3

s( 80.34\%)p 0.24( 19.66\%)d 0.00( $0.00 \%)$

s( $1.62 \%)$ p60.86( $98.30 \%) d 0.05(0.09 \%)$ 
10. (0.94698) LP ( 3 ) F $3 \quad$ s( $0.00 \%)$ p 1.00 ( 99.91\%)d $0.00(0.09 \%)$

11. (0.90563) LP (4) F $3 \quad$ s( $18.03 \%)$ p 4.54(81.81\%)d 0.01( $0.16 \%)$

12. (0.99987) BD ( 1) O 1- V 2

( 75.63\%) $0.8696 *$ O $1 \mathrm{~s}(11.52 \%) \mathrm{p} 7.66(88.23 \%) \mathrm{d} 0.02(0.25 \%)$

( $24.37 \%) \quad 0.4937 * \mathrm{~V} 2 \mathrm{~s}(2.56 \%) \mathrm{p} 0.23(0.58 \%) \mathrm{d} 37.77(96.62 \%)$ f $0.10(0.25 \%)$

13. (0.99976) BD (2) O 1- V 2

(81.12\%) $0.9007 *$ O $1 \mathrm{~s}(0.00 \%)$ p $1.00(99.76 \%) \mathrm{d} 0.00(0.24 \%)$

( $18.88 \%) \quad 0.4345 * \mathrm{~V} 2 \mathrm{~s}(0.00 \%) \mathrm{p} 1.00(0.38 \%) \mathrm{d} 99.99(99.49 \%)$ f $0.32(0.12 \%)$

14. (0.99947) BD ( 3) O 1- V 2

(82.04\%) $0.9058^{*} \mathrm{O} 1 \mathrm{~s}(0.88 \%) \mathrm{p} 99.99(98.88 \%) \mathrm{d} 0.27(0.24 \%)$

(17.96\%) $0.4237 * \mathrm{~V} 2 \mathrm{~s}(0.04 \%) \mathrm{p} 15.30(0.54 \%) \mathrm{d} 99.99(99.28 \%)$ f $4.07(0.14 \%)$

\begin{abstract}
${ }^{4} \mathrm{OCrF}$
Alpha

7. (0.99702) LP ( 1) O $1 \quad$ s( 81.36\%)p 0.23( $18.63 \%)$ d $0.00(0.01 \%)$

8. (0.99964) LP (1)Cr 2 s( 6.70\%)p 0.00( 0.01\%)d13.93(93.29\%) f $0.00(0.00 \%)$

9. (0.99816) LP (2)Cr 2 s( 0.00\%)p 1.00( 0.14\%)d99.99( 99.85\%) f $0.03(0.00 \%)$

10. (0.99235) LP (3)Cr $2 \quad$ s( 21.52\%)p 0.04( $0.90 \%) d 3.60(77.57 \%)$ f $0.00(0.01 \%)$

11. (0.99900) LP ( 1) F $3 \quad$ s( $76.51 \%)$ p 0.31(23.48\%)d 0.00( $0.01 \%)$

12. (0.96544) LP (2) F $3 \quad$ s( 9.42\%)p 9.61(90.48\%)d 0.01( $0.10 \%)$

13. (0.94201) LP (3) F $3 \quad$ s( $0.00 \%)$ p $1.00(99.89 \%)$ d $0.00(0.11 \%)$

14. (0.89548) LP (4) F $3 \quad$ s( $14.06 \%)$ p 6.10 ( 85.76\%)d 0.01( $0.18 \%)$

15. (0.98731) BD ( 1) O 1-Cr 2

(72.04\%) $0.8488^{*} \mathrm{O} 1 \mathrm{~s}(13.44 \%) \mathrm{p} 6.42(86.28 \%) \mathrm{d} 0.02(0.28 \%)$

(27.96\%) $0.5287^{*} \mathrm{Cr} 2 \mathrm{~s}(35.33 \%) \mathrm{p} 0.03(1.17 \%) \mathrm{d} 1.79(63.30 \%)$ f $0.01(0.21 \%)$
\end{abstract}

16. (0.99900) BD (2) O 1-Cr 2

(62.70\%) $0.7919 *$ O $1 \mathrm{~s}(0.00 \%) \mathrm{p} 1.00(99.67 \%) \mathrm{d} 0.00(0.33 \%)$

(37.30\%) $0.6107^{*} \mathrm{Cr} 2 \mathrm{~s}(0.00 \%) \mathrm{p} 1.00(0.61 \%) \mathrm{d} 99.99(99.35 \%)$ f $0.08(0.05 \%)$

17. (0.98702) BD (3) O 1-Cr 2

( 79.89\%) $0.8938^{*} \mathrm{O} 1 \mathrm{~s}(5.22 \%) \mathrm{p} 18.11(94.49 \%) \mathrm{d} 0.06(0.30 \%)$ ( $20.11 \%) \quad 0.4484 * \operatorname{Cr} 2 \mathrm{~s}(30.72 \%)$ p 0.14( 4.34\%)d 2.11( 64.82\%) f $0.00(0.12 \%)$

\title{
Beta
}

7. (0.98847) LP ( 1) O $1 \quad$ s( 87.55\%)p 0.14( $12.45 \%)$ d $0.00(0.00 \%)$

8. (0.99725) LP ( 1) F $3 \quad$ s( 78.17\%)p 0.28(21.83\%)d 0.00( $0.01 \%)$

9. (0.95652) LP (2) F $3 \quad$ s( $3.44 \%)$ p28.03( 96.46\%)d $0.03(0.10 \%)$

10. (0.94325) LP (3) F $3 \quad$ s( $0.00 \%)$ p $1.00(99.89 \%)$ d $0.00(0.11 \%)$ 
11. (0.99409) BD ( 1) O 1-Cr 2

(77.54\%) $0.8806^{*} \mathrm{O} 1 \mathrm{~s}(9.06 \%) \mathrm{p} 10.00(90.65 \%) \mathrm{d} 0.03(0.28 \%)$

( $22.46 \%) \quad 0.4739^{*} \mathrm{Cr} 2 \mathrm{~s}(6.78 \%) \mathrm{p} 0.08(0.52 \%) \mathrm{d} 13.64(92.43 \%)$ f $0.04(0.27 \%)$

12. (0.99972) BD (2) O 1-Cr 2

( 80.65\%) $0.8981 *$ O $1 \mathrm{~s}(0.00 \%) \mathrm{p} 1.00(99.72 \%) \mathrm{d} 0.00(0.28 \%)$

( $19.35 \%) \quad 0.4398^{*} \mathrm{Cr} 2 \mathrm{~s}(0.00 \%) \mathrm{p} 1.00(0.42 \%) \mathrm{d} 99.99(99.47 \%)$ f $0.28(0.12 \%)$

13. (0.97354) BD (3) O 1-Cr 2

( $85.12 \%) \quad 0.9226 *$ O $1 \mathrm{~s}(3.39 \%) p 28.40(96.33 \%) d 0.08(0.28 \%)$

( $14.88 \%) \quad 0.3858 * \mathrm{Cr} 2 \mathrm{~s}(3.75 \%) \mathrm{p} 0.57(2.14 \%) \mathrm{d} 24.94(93.61 \%)$ f $0.13(0.49 \%)$

14. (0.97874) BD ( 1)Cr 2- F 3

( $7.43 \%) \quad 0.2726 * \mathrm{Cr} 2 \mathrm{~s}(31.89 \%)$ p 0.07( $2.11 \%) \mathrm{d} 2.05(65.33 \%)$ f $0.02(0.66 \%)$

(92.57\%) $0.9621 *$ F 3 s( 18.37\%)p 4.43( 81.45\%)d 0.01( 0.18\%)

\section{${ }^{5} \mathrm{OMnF}$ \\ Alpha}

7. (0.99730) LP ( 1) O $1 \quad$ s( 87.38\%)p 0.14( 12.61\%)d 0.00( $0.01 \%)$

8. (0.98873) LP (2) O 1 s( $0.00 \%)$ p $1.00(99.74 \%)$ d $0.00(0.26 \%)$

9. (0.99959) LP (1)Mn 2 s( 4.93\%)p 0.00( 0.02\%)d19.27(95.04\%) f $0.00(0.00 \%)$

10. (0.99862) LP (2)Mn $2 \quad$ s( $0.00 \%)$ p 1.00( 0.08\%)d99.99( 99.92\%) f $0.07(0.01 \%)$

11. (0.99449) LP (3)Mn $2 \quad$ s( $0.00 \%)$ p 1.00( 0.27\%)d99.99( 99.73\%) f $0.01(0.00 \%)$

12. (0.99358) LP (4)Mn $2 \quad$ s( $13.80 \%)$ p 0.06( $0.88 \%) d$ 6.18( 85.32\%) f $0.00(0.00 \%)$

13. (0.99915) LP ( 1) F $3 \quad$ s( $83.46 \%)$ p 0.20 ( $16.54 \%)$ d $0.00(0.01 \%)$ 14. (0.99743) LP (2) F $3 \quad$ s( $0.00 \%)$ p $1.00(99.92 \%)$ d $0.00(0.08 \%)$ 15. (0.97141) LP (3) F $3 \quad$ s( $6.78 \%)$ p13.73(93.13\%)d 0.01( $0.09 \%)$ 16. (0.87643) LP (4) F 3 s( 9.74\%)p 9.25(90.06\%)d 0.02( $0.21 \%)$

17. (0.97314) BD ( 1) O 1-Mn 2

(61.85\%) $\quad 0.7865 *$ O $1 \mathrm{~s}(7.46 \%) p 12.34(92.11 \%) \mathrm{d} 0.06(0.43 \%)$

(38.15\%) $0.6176^{*} \mathrm{Mn} 2 \mathrm{~s}(29.48 \%)$ p 0.05 ( 1.47\%)d 2.34( 68.92\%) f $0.00(0.12 \%)$

18. (0.97520) BD ( 2) O 1-Mn 2

(78.22\%) $\quad 0.8844 * \mathrm{O} 1 \mathrm{~s}(5.17 \%) \mathrm{p} 18.27(94.52 \%) \mathrm{d} 0.06(0.31 \%)$

( $21.78 \%) \quad 0.4667 * \operatorname{Mn} 2 \mathrm{~s}(43.39 \%)$ p $0.15(6.52 \%) d 1.15(49.97 \%)$ f $0.00(0.12 \%)$

\section{Beta}

7. (0.99057) LP ( 1) O 1

8. (0.99779) LP ( 1) F 3 s( 90.82\%)p 0.10( 9.17\%)d 0.00( $0.00 \%)$

s( $84.24 \%)$ p 0.19( $15.76 \%) d 0.00(0.00 \%)$ 
9. (0.95256) LP ( 2) F 3

10. (0.93774) LP ( 3$)$ F 3

11. (0.89074) LP ( 4) F 3

12. (0.99972) BD ( 1) O 1-Mn 2

( 74.56\%) $0.8635^{*} \mathrm{O} 1 \mathrm{~s}(6.49 \%) \mathrm{p} 14.34(93.14 \%) \mathrm{d} 0.06(0.37 \%)$

( $25.44 \%) \quad 0.5044 * \operatorname{Mn} 2 \mathrm{~s}(3.10 \%) \mathrm{p} 0.07(0.21 \%) \mathrm{d} 31.16(96.54 \%)$ f $0.05(0.15 \%)$

13. (0.99984) BD (2) O 1-Mn 2

( 76.03\%) $0.8720 *$ O $1 \mathrm{~s}(0.00 \%) \mathrm{p} 1.00(99.68 \%) \mathrm{d} 0.00(0.32 \%)$

(23.97\%) $0.4895^{*} \mathrm{Mn} 2 \mathrm{~s}(0.00 \%) \mathrm{p} 1.00(0.77 \%) \mathrm{d} 99.99(99.12 \%)$ f $0.15(0.12 \%)$

14. (0.99935) BD ( 3) O 1-Mn 2

( 78.53\%) $0.8862 * \mathrm{O} 1 \mathrm{~s}(2.66 \%) \mathrm{p} 36.49(97.01 \%) \mathrm{d} 0.12(0.33 \%)$

(21.47\%) $0.4634 * \mathrm{Mn} 2 \mathrm{~s}(0.74 \%) \mathrm{p}$ 0.95( 0.70\%)d99.99( 98.42\%) f $0.19(0.14 \%)$

\section{${ }^{6} \mathrm{OFeF}$}

\section{Alpha}

7. (0.99909) LP ( 1) O 1

8. (0.99090) LP (2) O 1

9. (0.99038) LP (3) O 1

10. (0.99979) LP ( 1)Fe 2

11. (0.99975) LP ( 2)Fe 2

12. (0.99751) LP ( 3)Fe 2

13. (0.99725) LP ( 4)Fe 2

14. (0.99687) LP ( 5)Fe 2

15. (0.99955) LP ( 1) F 3

16. (0.99677) LP ( 2) F 3

17. (0.99664) LP ( 3 ) F 3

18. (0.92734) LP ( 4) F 3

19. (0.99950) BD ( 1) O 1-Fe 2

(84.29\%) $0.9181 *$ O $1 \mathrm{~s}(13.00 \%)$ p $6.67(86.68 \%) d 0.02(0.32 \%)$

( $15.71 \%) \quad 0.3963 * \mathrm{Fe} 2 \mathrm{~s}(82.60 \%) \mathrm{p} 0.02(1.67 \%) \mathrm{d} 0.19(15.59 \%)$ f 0.00 ( $0.14 \%)$

\section{Beta}

7. (0.98728) LP ( 1) O 1

8. (0.99824) LP ( 1) F 3

9. (0.94390) LP ( 2) F 3

10. (0.93042) LP ( 3$) \mathrm{F} 3$

11. (0.98740) BD (1) O 1-Fe 2 s( 82.17\%)p 0.22( $17.82 \%) d 0.00(0.01 \%)$ s( $4.79 \%) p 19.80(94.92 \%) d 0.06(0.28 \%)$ s( $0.00 \%) p 1.00(99.70 \%) d 0.00(0.30 \%)$ s( $0.19 \%) p$ p.07( $0.01 \%) d 99.99(99.79 \%)$ f $0.01(0.00 \%)$ f $0.08(0.00 \%)$ s( $9.69 \%)$ p 0.01( $0.09 \%) d$ 9.31( 90.22\%) f $0.00(0.00 \%)$ f $0.01(0.00 \%)$ s( $5.01 \%)$ p 0.04( $0.22 \%) \mathrm{d} 18.91(94.77 \%)$ f $0.00(0.00 \%)$ s( $83.33 \%)$ p $0.20(16.66 \%) d 0.00(0.00 \%)$ s( $0.00 \%)$ p $1.00(99.91 \%) d 0.00(0.09 \%)$ s( $1.72 \%)$ p57.02( 98.18\%)d $0.06(0.10 \%)$ s( 14.95\%)p 5.68( 84.87\%)d 0.01( 0.19\%)

\begin{abstract}
s( 94.77\%)p 0.06( 5.23\%)d 0.00( $0.00 \%)$ s( $84.55 \%)$ p 0.18( $15.45 \%) d 0.00(0.00 \%)$ s( $5.72 \%)$ p16.45( $94.15 \%) d 0.02(0.13 \%)$ s( $0.00 \%)$ p $1.00(99.88 \%) d 0.00(0.12 \%)$
\end{abstract}


(64.97\%) $0.8060 *$ O 1 s( 2.47\%)p39.31( 97.02\%)d 0.21( 0.51\%) (35.03\%) $0.5919 * \mathrm{Fe} 2 \mathrm{~s}(6.29 \%) \mathrm{p} 0.14(0.88 \%) \mathrm{d} 14.75(92.74 \%)$ f $0.01(0.09 \%)$

12. (0.99956) BD (2) O 1-Fe 2

(59.23\%) $0.7696 *$ O $1 \mathrm{~s}(0.00 \%)$ p $1.00(99.57 \%) \mathrm{d} 0.00(0.43 \%)$

( 40.77\%) $0.6385 * \mathrm{Fe} 2 \mathrm{~s}(0.00 \%) \mathrm{p} 1.00(0.78 \%) \mathrm{d} 99.99(99.18 \%)$ f $0.05(0.04 \%)$

13. (0.95404) BD ( 3 ) O 1-Fe 2

( 73.66\%) $0.8583 *$ O 1 s( $2.72 \%) p 35.62(96.82 \%) d 0.17(0.46 \%)$

( $26.34 \%) \quad 0.5132 * \mathrm{Fe} 2 \mathrm{~s}(2.81 \%) \mathrm{p} 1.26(3.55 \%) \mathrm{d} 33.17(93.35 \%)$ f $0.10(0.29 \%)$

14. (0.97812) BD ( 1)Fe 2- F 3

( $7.90 \%) \quad 0.2810 * \mathrm{Fe} 2 \mathrm{~s}(34.56 \%) \mathrm{p} 0.16(5.49 \%) \mathrm{d} 1.72(59.51 \%)$ f $0.01(0.44 \%)$

(92.10\%) $0.9597 *$ F 3 s( 9.72\%)p 9.27( 90.07\%)d 0.02( 0.21\%)

\section{${ }^{5} \mathrm{OCoF}$ \\ Alpha}

7. (0.99926) LP ( 1) O $1 \quad$ s( 83.65\%)p 0.20( 16.35\%)d 0.00( $0.00 \%)$

8. (0.99332) LP (2) O 1 s( $0.00 \%)$ p $1.00(99.73 \%) d 0.00(0.27 \%)$

9. (0.99332) LP (3) O 1 s( $0.00 \%)$ p $1.00(99.73 \%)$ d $0.00(0.27 \%)$

10. (0.99997) LP ( 1)Co 2 s( $0.00 \%)$ p 0.00( $0.00 \%)$ d $1.00(100.00 \%)$ f $0.00(0.00 \%)$

11. (0.99995) LP (2)Co 2 s( $0.00 \%)$ p 0.00( $0.00 \%)$ d $1.00(100.00 \%)$ f $0.00(0.00 \%)$

12. (0.99770) LP (3)Co 2 s( $0.00 \%)$ p 1.00( 0.03\%)d99.99( 99.97\%) f $0.00(0.00 \%)$

13. (0.99770) LP (4)Co 2 s( $0.00 \%)$ p 1.00( $0.03 \%) \mathrm{d} 99.99(99.97 \%)$ f $0.00(0.00 \%)$

14. (0.99748) LP (5)Co $2 \quad$ s( $11.82 \%)$ p 0.00( 0.00\%)d 7.46( 88.18\%) f $0.00(0.00 \%)$

15. (0.99962) LP ( 1) F $3 \quad$ s( $84.33 \%)$ p $0.19(15.67 \%) d 0.00(0.00 \%)$ 16. (0.99687) LP (2) F $3 \quad$ s( $0.00 \%)$ p $1.00(99.90 \%) d 0.00(0.10 \%)$ 17. (0.99687) LP (3) F $3 \quad$ s( $0.00 \%)$ p $1.00(99.90 \%)$ d $0.00(0.10 \%)$ 18. (0.92575) LP (4) F $3 \quad$ s( $15.67 \%)$ p 5.37( 84.12\%)d 0.01( $0.21 \%)$ 19. (0.99979) BD ( 1) O 1-Co 2

(86.96\%) $0.9325^{*}$ O $1 \mathrm{~s}(16.31 \%)$ p 5.11( 83.37\%)d 0.02( $\left.0.32 \%\right)$ ( $13.04 \%) \quad 0.3611^{*}$ Co 2 s( $\left.87.18 \%\right)$ p $0.01(0.45 \%) d$ 0.14( $\left.12.22 \%\right)$ f $0.00(0.15 \%)$

\section{Beta}

7. (0.98579) LP ( 1) O 1

8. (0.99999) LP ( 1)Co 2

9. (0.99823) LP ( 1) F 3

10. (0.93211) LP ( 2) F 3 s( $96.07 \%)$ p $0.04(3.93 \%) d 0.00(0.01 \%)$

s( $0.00 \%)$ p $0.00(0.00 \%) d 1.00(100.00 \%)$ f $0.00(0.00 \%)$

s( $85.25 \%)$ p $0.17(14.75 \%) d 0.00(0.00 \%)$

s( $0.00 \%)$ p $1.00(99.88 \%) d 0.00(0.12 \%)$ 
11. (0.93211) LP ( 3 ) F $3 \quad$ s( $0.00 \%)$ p 1.00 ( 99.88\%)d $0.00(0.12 \%)$

12. (0.91812) LP (4) F $3 \quad \mathrm{~s}(14.75 \%)$ p 5.77( 85.02\%)d 0.02( $0.23 \%)$

13. (0.99943) BD ( 1) O 1-Co 2

(53.93\%) $0.7343 *$ O $1 \mathrm{~s}(0.00 \%) \mathrm{p} 1.00(99.57 \%) \mathrm{d} 0.00(0.43 \%)$

( 46.07\%) $0.6788 *$ Co 2 s( $0.00 \%)$ p 1.00( 0.71\%)d $99.99(99.27 \%)$ f $0.04(0.03 \%)$

14. (0.99943) BD (2) O 1-Co 2

(53.93\%) $0.7343 *$ O $1 \mathrm{~s}(0.00 \%) \mathrm{p} 1.00(99.57 \%) \mathrm{d} 0.00(0.43 \%)$

( 46.07\%) $0.678 *^{*}$ Co 2 s( $\left.0.00 \%\right)$ p $1.00(0.71 \%) \mathrm{d} 99.99(99.27 \%)$ f $0.04(0.03 \%)$

15. (0.99907) BD ( 3) O 1-Co 2

(68.81\%) $0.8295 *$ O $1 \mathrm{~s}(3.88 \%)$ p24.61( $95.51 \%) d 0.16(0.61 \%)$

(31.19\%) $0.5585^{*}$ Co 2 s( $\left.2.10 \%\right)$ p $0.05(0.11 \%) d 46.60(97.72 \%)$ f $0.04(0.07 \%)$

\footnotetext{
${ }^{4} \mathrm{ONiF}$

Alpha

7. (0.99942) LP ( 1) O $1 \quad$ s( 81.59\%)p 0.23( $18.40 \%) d 0.00(0.01 \%)$

8. (0.99546) LP (2) O 1 s( $0.00 \%)$ p $1.00(99.77 \%)$ d $0.00(0.23 \%)$

9. (0.99546) LP (3) O 1 s( $0.00 \%)$ p $1.00(99.77 \%)$ d $0.00(0.23 \%)$

10. (0.99999) LP( 1$) \mathrm{Ni} 2$ s( $0.00 \%)$ p $0.00(0.00 \%) \mathrm{d} 1.00(100.00 \%)$ f $0.00(0.00 \%)$

11. (0.99999) LP (2)Ni $2 \quad$ s( $0.00 \%)$ p $0.00(0.00 \%) d 1.00(100.00 \%)$ f $0.00(0.00 \%)$

12. (0.99791) LP (3)Ni 2 s( $0.00 \%)$ p $1.00(0.02 \%) \mathrm{d} 99.99(99.98 \%)$ f $0.01(0.00 \%)$

13. (0.99791) LP(4)Ni 2 s( $0.00 \%)$ p 1.00( $0.02 \%) \mathrm{d} 99.99(99.98 \%)$ f $0.01(0.00 \%)$

14. (0.99712) LP (5)Ni $2 \quad$ s( 9.18\%)p 0.00( $0.00 \%) d$ 9.89(90.81\%) f $0.00(0.00 \%)$

15. (0.99967) LP ( 1) F $3 \quad$ s( $83.50 \%)$ p $0.20(16.50 \%) d 0.00(0.00 \%)$

16. (0.99724) LP (2) F $3 \quad$ s( $0.00 \%)$ p $1.00(99.90 \%) d 0.00(0.10 \%)$

17. (0.99724) LP (3) F $3 \quad$ s( $0.00 \%)$ p $1.00(99.90 \%) d 0.00(0.10 \%)$

18. (0.92947) LP (4) F 3 s( $16.50 \%)$ p 5.05( 83.27\%)d 0.01( $0.23 \%)$

19. (0.99984) BD ( 1) O 1-Ni 2

(89.00\%) $\quad 0.9434 *$ O $1 \mathrm{~s}(18.37 \%)$ p $4.43(81.31 \%) d 0.02(0.32 \%)$

( $11.00 \%) \quad 0.3317 * \mathrm{Ni} 2 \mathrm{~s}(89.88 \%)$ p 0.01( 0.54\%)d 0.10( 9.40\%) f $0.00(0.17 \%)$
}

\section{Beta}

7. (0.98546) LP ( 1) O 1

8. (0.99999) LP ( 1)Ni 2

s( $97.25 \%)$ p $0.03(2.74 \%) d 0.00(0.01 \%)$

9. (0.99999) LP ( 2)Ni 2

s( $0.00 \%)$ p $0.00(0.00 \%) d 1.00(100.00 \%)$ f $0.00(0.00 \%)$

s( $0.00 \%)$ p $0.00(0.00 \%) d 1.00(100.00 \%)$

f $0.00(0.00 \%)$ 
10. (0.99789) LP ( 1) F 3

s( 87.44\%)p 0.14( $12.56 \%) d 0.00(0.00 \%)$

11. (0.92045) LP ( 2) F 3

s( $0.00 \%)$ p $1.00(99.87 \%)$ d $0.00(0.13 \%)$

12. $(0.92045) \mathrm{LP}$ ( 3$) \mathrm{F} 3$

s( $0.00 \%)$ p $1.00(99.87 \%)$ d $0.00(0.13 \%)$

13. (0.90810) LP ( 4) F 3

14. (0.99938) BD ( 1) O 1-Ni 2

( 48.20\%) $0.6943 *$ O $1 \mathrm{~s}(0.00 \%)$ p $1.00(99.62 \%) d 0.00(0.38 \%)$

( $51.80 \%) \quad 0.7197 * \mathrm{Ni} 2 \mathrm{~s}(0.00 \%)$ p $1.00(0.57 \%) \mathrm{d} 99.99(99.41 \%)$ f $0.03(0.02 \%)$

15. (0.99938) BD (2) O 1-Ni 2

s( $12.55 \%)$ p 6.95( $87.20 \%) d 0.02(0.26 \%)$

( 48.20\%) $0.6943 *$ O $1 \mathrm{~s}(0.00 \%)$ p $1.00(99.62 \%) \mathrm{d} 0.00(0.38 \%)$

(51.80\%) $0.7197 * \mathrm{Ni} 2 \mathrm{~s}(0.00 \%)$ p $1.00(0.57 \%) \mathrm{d} 99.99(99.41 \%)$ f $0.03(0.02 \%)$

16. (0.99905) BD ( 3) O 1-Ni 2

(63.78\%) $0.7986^{*} \mathrm{O} 1 \mathrm{~s}(2.72 \%) \mathrm{p} 35.59(96.63 \%) \mathrm{d} 0.24(0.65 \%)$

(36.22\%) $0.6018^{*} \mathrm{Ni} 2 \mathrm{~s}(1.82 \%) \mathrm{p} 0.06(0.11 \%) \mathrm{d} 53.86(98.01 \%)$ f $0.03(0.06 \%)$

\author{
${ }^{3} \mathrm{OCuF}$ \\ Alpha \\ 7. (0.99965) LP ( 1) O 1 \\ s( 83.01\%)p 0.20( $16.96 \%) d 0.00(0.02 \%)$ \\ 8. (0.99735) LP ( 2) O 1 \\ s( $0.00 \%)$ p $1.00(99.81 \%) d 0.00(0.19 \%)$ \\ 9. (0.99735) LP ( 3) O 1 \\ 10. (0.99999) LP ( 1)Cu 2 \\ s( $0.00 \%)$ p $1.00(99.81 \%)$ d $0.00(0.19 \%)$ \\ s( $0.00 \%)$ p $0.00(0.00 \%) d 1.00(100.00 \%)$ \\ f $0.00(0.00 \%)$ \\ 11. (0.99999) LP ( 2)Cu 2 \\ 12. (0.99875) LP ( 3) Cu 2 \\ s( $0.00 \%)$ p $0.00(0.00 \%) d 1.00(100.00 \%)$ \\ f $0.00(0.00 \%)$ \\ 13. (0.99875) LP (4)Cu 2 \\ s( $0.00 \%)$ p $0.00(0.00 \%) d 1.00(100.00 \%)$ \\ f $0.00(0.00 \%)$ \\ 14. (0.99715) LP ( 5)Cu 2 \\ s( $0.00 \%)$ p $0.00(0.00 \%) d 1.00(100.00 \%)$ \\ f $0.00(0.00 \%)$ \\ s( 8.31\%)p 0.00( 0.00\%)d11.04( 91.69\%) \\ 15. (0.99972) LP ( 1) F 3 \\ f $0.00(0.00 \%)$ \\ 16. (0.99729) LP ( 2) F 3 \\ s( $83.34 \%)$ p 0.20 ( $16.66 \%) d 0.00(0.01 \%)$ \\ s( $0.00 \%)$ p 1.00 ( 99.89\%)d $0.00(0.11 \%)$ \\ 17. (0.99729) LP ( 3$) \mathrm{F} 3$ \\ s( $0.00 \%)$ p $1.00(99.89 \%)$ d $0.00(0.11 \%)$ \\ 18. (0.91782) LP ( 4) F 3 \\ s( $16.70 \%) p$ 4.98( $83.08 \%) d 0.01(0.22 \%)$ \\ 19. (0.99961) BD ( 1) O 1-Cu 2 \\ (88.08\%) $0.9385^{*} \mathrm{O} 1 \mathrm{~s}(17.02 \%) \mathrm{p} 4.86(82.69 \%) \mathrm{d} 0.02(0.29 \%)$ \\ ( $11.92 \%) \quad 0.3453 * \mathrm{Cu} 2 \mathrm{~s}(90.85 \%)$ p 0.01( $0.89 \%) \mathrm{d} 0.09(8.14 \%)$ \\ f $0.00(0.11 \%)$
}

\title{
Beta
}

7. (0.99960) LP ( 1) O $1 \quad$ s( 82.96\%)p 0.21( $17.04 \%) d 0.00(0.00 \%)$

8. (0.99999) LP ( 1)Cu 2 s( $0.00 \%)$ p 0.00( $0.00 \%) d 1.00(100.00 \%)$

9. (1.00000) LP (2)Cu $2 \quad$ s( $0.00 \%)$ p 0.00( $0.00 \%) d 1.00(100.00 \%)$ 


$$
\begin{aligned}
& \text { f } 0.00(0.00 \%) \\
& \text { 10. (0.99679) LP (3)Cu } 2 \text { s( 9.42\%)p 0.00( 0.00\%)d 9.62( 90.58\%) } \\
& \text { f } 0.00(0.00 \%) \\
& \text { 11. (0.99973) LP ( 1) F } 3 \quad \text { s( 82.88\%)p 0.21( } 17.12 \%) d 0.00(0.00 \%) \\
& \text { 12. (0.91850) LP ( 2) F } 3 \quad s(17.16 \%) \text { p 4.82( } 82.62 \%) d 0.01(0.22 \%) \\
& \text { 13. (0.91130) LP ( 3) F } 3 \quad \text { s( } 0.00 \%) \text { p } 1.00(99.87 \%) d 0.00(0.13 \%) \\
& \text { 14. (0.91130) LP ( 4) F } 3 \quad \text { s( } 0.00 \%) \text { p 1.00( 99.87\%)d 0.00( } 0.13 \%) \\
& \text { 15. (0.99952) BD ( 1) O 1-Cu } 2
\end{aligned}
$$

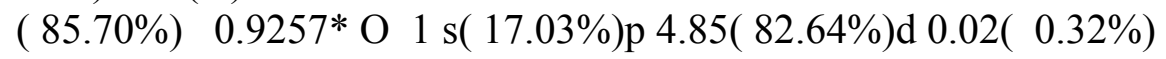

$$
\begin{aligned}
& (14.30 \%) \quad 0.3782 * \mathrm{Cu} 2 \text { s( 89.49\%)p 0.01( 1.19\%)d 0.10( 9.21\%) } \\
& \text { f } 0.00(0.10 \%)
\end{aligned}
$$

16. (0.99929) BD ( 2) O 1-Cu 2

$$
\begin{array}{ll}
(30.92 \%) & 0.5560 * \mathrm{O} 1 \mathrm{~s}(0.00 \%) \mathrm{p} 1.00(99.72 \%) \mathrm{d} 0.00(0.28 \%) \\
(69.08 \%) & 0.8312 * \mathrm{Cu} 2 \mathrm{~s}(0.00 \%) \mathrm{p} 1.00(0.32 \%) \mathrm{d} 99.99(99.67 \%)
\end{array}
$$
f $0.01(0.00 \%)$

17. (0.99929) BD ( 3) O 1-Cu 2

(30.92\%) $0.5560 *$ O 1 s( $0.00 \%)$ p 1.00 (99.72\%)d 0.00( $0.28 \%)$

(69.08\%) $0.8312 * \mathrm{Cu} 2 \mathrm{~s}(0.00 \%) \mathrm{p} 1.00(0.32 \%) \mathrm{d} 99.99(99.67 \%)$ f $0.01(0.00 \%)$ 


\section{${ }^{2} \mathrm{OScF}_{2}$ \\ Alpha}

8. (0.99483) LP ( 1) O 1

s( 74.50\%)p 0.34( 25.49\%)d 0.00( $0.01 \%)$

9. (0.93976) LP ( 2) O 1

s( $0.00 \%)$ p $1.00(99.87 \%) d 0.00(0.13 \%)$

10. (0.99517) LP ( 1) F 3

s( 79.57\%)p 0.26( 20.43\%)d 0.00( $0.01 \%)$

11. (0.96497) LP ( 2) F 3

s( $0.17 \%)$ p99.99( $99.73 \%) d 0.59(0.10 \%)$

12. (0.94957) LP ( 3) F 3

s( $0.00 \%)$ p $1.00(99.88 \%) d 0.00(0.12 \%)$

13. (0.99517) LP ( 1) F 4

s( 79.57\%)p 0.26( 20.43\%)d 0.00( 0.01\%)

14. (0.96497) LP ( 2) F 4

s( $0.17 \%)$ p99.99( $99.73 \%) d 0.59(0.10 \%)$

15. (0.94957) LP ( 3) F 4

16. (0.99967) BD ( 1) O 1-Sc 2

s( $0.00 \%)$ p 1.00( 99.88\%)d 0.00( $0.12 \%)$

(91.08\%) $0.9544 *$ O $1 \mathrm{~s}(0.00 \%)$ p 1.00 ( 99.84\%)d 0.00( $0.16 \%)$

( $8.92 \%) \quad 0.2986 *$ Sc $2 \mathrm{~s}(0.00 \%) \mathrm{p} 1.00(0.95 \%) \mathrm{d} 99.99(98.81 \%)$

f $0.25(0.24 \%)$

17. (0.99958) BD ( 2) O 1-Sc 2

(90.83\%) $0.9530 *$ O 1 s( $25.39 \%)$ p 2.93( 74.39\%)d 0.01( $0.22 \%)$

( 9.17\%) $0.3028 *$ Sc 2 s( 6.01\%)p 0.01( 0.09\%)d15.55(93.48\%) f $0.07(0.43 \%)$

18. (0.99958) BD ( 1)Sc 2- F 3

$$
\text { ( } 0.07(0.43 \%)
$$

( 7.71\%) $0.2777 *$ Sc 2 s( 8.14\%)p 0.01( 0.09\%)d11.26( 91.67\%) f $0.01(0.10 \%)$

(92.29\%) $0.9607 *$ F 3 s( 20.25\%)p 3.93( 79.59\%)d 0.01( $0.16 \%)$ 19. (0.99958) BD ( 1)Sc 2-F 4

( 7.71\%) $0.2777 *$ Sc 2 s( 8.14\%)p 0.01( 0.09\%)d11.26( 91.67\%) f $0.01(0.10 \%)$

( 92.29\%) $0.9607 *$ F 4 s( 20.25\%)p 3.93( 79.59\%)d 0.01( $0.16 \%)$

\section{Beta}

8. (0.96638) LP ( 1) O $1 \quad$ s( 99.61\%)p 0.00( $0.38 \%) d 0.00(0.01 \%)$

9. (0.92832) LP ( 2) O $1 \quad \mathrm{~s}(0.00 \%)$ p $1.00(99.84 \%) \mathrm{d} 0.00(0.16 \%)$

10. (0.99466) LP ( 1) F $3 \quad$ s( 80.52\%)p 0.24( $19.48 \%) d 0.00(0.01 \%)$

11. (0.94973) LP ( 2) F $3 \quad s(0.00 \%)$ p $1.00(99.88 \%) d 0.00(0.12 \%)$

12. (0.94251) LP ( 3) F $3 \quad$ s( $6.02 \%)$ p15.60(93.87\%)d 0.02( $0.11 \%)$

13. (0.99466) LP ( 1) F $4 \quad \mathrm{~s}(80.52 \%)$ p 0.24( $19.48 \%) \mathrm{d} 0.00(0.01 \%)$

14. (0.94973) LP ( 2) F $4 \quad s(0.00 \%)$ p $1.00(99.88 \%) d 0.00(0.12 \%)$

15. (0.94251) LP ( 3) F $4 \quad$ s( 6.02\%)p15.60(93.87\%)d 0.02( $0.11 \%)$

16. (0.99940) BD ( 1) O 1-Sc 2

$(89.13 \%) \quad 0.9441 *$ O 1 s( $0.00 \%)$ p $1.00(99.79 \%) d 0.00(0.21 \%)$

(10.87\%) $0.3297 *$ Sc 2 s( 0.00\%)p 1.00( 1.01\%)d $97.51(98.84 \%)$ f $0.15(0.15 \%)$

17. (0.98477) BD ( 1)Sc 2- F 3

( 7.18\%) $0.2680 *$ Sc 2 s( 10.36\%)p 0.02( 0.24\%)d 8.62( 89.27\%) f $0.01(0.14 \%)$

(92.82\%) $0.9634 * \mathrm{~F} 3 \mathrm{~s}(13.45 \%) \mathrm{p}$ 6.42( 86.39\%)d 0.01( $0.15 \%)$

18. (0.98477) BD ( 1)Sc 2- F 4

( 7.18\%) $0.2680 *$ Sc 2 s( 10.36\%)p 0.02( 0.24\%)d 8.62( 89.27\%) 


$$
\text { f } 0.01(0.14 \%)
$$

( $92.82 \%) \quad 0.9634 *$ F 4 s( $13.45 \%)$ p 6.42( 86.39\%)d 0.01( $0.15 \%)$

\section{${ }^{1} \mathrm{OTiF}_{2}$}

5. (1.98441) CR (4)Ti 2

8. (1.97842) LP ( 1) O 1

9. (1.99202) LP ( 1) F 3

10. (1.92655) LP ( 2) F 3

11. (1.86147) LP ( 3) F 3

12. (1.99202) LP ( 1) F 4

13. (1.92655) LP ( 2) F 4 s( $0.00 \%)$ p $1.00(100.00 \%)$

s( $89.45 \%)$ p $0.12(10.55 \%) d 0.00(0.00 \%)$ s( $81.96 \%)$ p 0.22( $18.04 \%) d 0.00(0.00 \%)$ s( $0.25 \%) p 99.99(99.67 \%) d 0.35(0.08 \%)$ s( $0.00 \%)$ p 1.00( 99.88\%)d $0.00(0.12 \%)$ s( $81.96 \%)$ p $0.22(18.04 \%) d 0.00(0.00 \%)$ s( $0.25 \%) p 99.99(99.67 \%) d 0.35(0.08 \%)$ $-0.0001-0.0193-0.0003$

14. (1.86147) LP ( 3) F 4 15. (1.99894) BD ( 1) O 1-Ti 2 s( $0.00 \%) p 1.00(99.88 \%) d 0.00(0.12 \%)$

( 76.16\%) $0.8727 *$ O $1 \mathrm{~s}(0.00 \%)$ p $1.00(99.70 \%) d 0.00(0.30 \%)$ ( $23.84 \%) \quad 0.4882 *$ Ti 2 s( $0.00 \%) p$ 1.00( 0.44\%)d99.99( $99.39 \%)$ f $0.38(0.17 \%)$

16. (1.99314) BD (2) O 1-Ti 2

( 74.23\%) $0.8616^{*} \mathrm{O} 1 \mathrm{~s}(10.61 \%) \mathrm{p} 8.40(89.10 \%) \mathrm{d} 0.03(0.30 \%)$

( $25.77 \%) \quad 0.5076 *$ Ti 2 s( $1.94 \%)$ p 0.10( $0.20 \%) d 50.17(97.53 \%)$ f $0.17(0.33 \%)$

17. (1.90381) BD (3) O 1-Ti 2

( 87.02\%) $0.9329 *$ O $1 \mathrm{~s}(0.00 \%)$ p $1.00(99.76 \%) \mathrm{d} 0.00(0.24 \%)$

( $12.98 \%) \quad 0.3602 * \mathrm{Ti} 2 \mathrm{~s}(0.00 \%)$ p $1.00(30.29 \%) \mathrm{d} 2.18(66.06 \%)$ f $0.12(3.65 \%)$

18. (1.93838) BD ( 1)Ti 2- F 3

( 6.88\%) $0.2623 *$ Ti $2 \mathrm{~s}(6.16 \%) \mathrm{p} 4.73(29.14 \%) \mathrm{d} 9.97(61.38 \%)$ f $0.54(3.32 \%)$

(93.12\%) $0.9650 * \mathrm{~F} 3 \mathrm{~s}(17.79 \%)$ p 4.61( 82.05\%)d 0.01( 0.16\%) 19. (1.93838) BD ( 1)Ti 2- F 4

( 6.88\%) $0.2623 * \mathrm{Ti} 2 \mathrm{~s}(6.16 \%) \mathrm{p} 4.73(29.14 \%) \mathrm{d} 9.97(61.38 \%)$ f $0.54(3.32 \%)$

( 93.12\%) $0.9650 *$ F 4 s( $17.79 \%)$ p 4.61( 82.05\%)d 0.01( 0.16\%)

\section{${ }^{2} \mathrm{OVF}_{2}$ \\ Alpha}

8. (0.98939) LP ( 1) O 1

9. (0.78791) LP ( 2) O 1

10. (0.99680) LP ( 1) V 2

11. (0.99629) LP ( 1) F 3

12. (0.95449) LP ( 2) F 3

13. (0.99629) LP ( 1) F 4

14. (0.95449) LP ( 2) F 4

15. (0.98536) BD ( 1) O 1- V 2 s( $87.75 \%)$ p 0.14( $12.25 \%)$ d $0.00(0.00 \%)$ s( $0.00 \%)$ p 1.00 ( 99.71\%)d $0.00(0.29 \%)$ s( 9.79\%)p 0.00( $0.00 \%) d$ 9.21( 90.19\%) f $0.00(0.01 \%)$ s( $79.73 \%)$ p $0.25(20.27 \%)$ d $0.00(0.00 \%)$ s( $0.00 \%)$ p $1.00(99.89 \%) d 0.00(0.10 \%)$ s( $79.73 \%) p 0.25(20.27 \%) d 0.00(0.00 \%)$ s( $0.00 \%)$ p $1.00(99.89 \%) d 0.00(0.10 \%)$ 
(72.96\%) $0.8542 * \mathrm{O} 1 \mathrm{~s}(12.37 \%) \mathrm{p} 7.07(87.37 \%) \mathrm{d} 0.02(0.26 \%)$

(27.04\%) $0.5200 * \mathrm{~V} 2 \mathrm{~s}(20.48 \%) \mathrm{p} 0.00(0.06 \%) \mathrm{d} 3.86(79.03 \%)$ f $0.02(0.43 \%)$

16. (0.97173) BD (2) O 1- V 2

( 72.10\%) $0.8491 *$ O $1 \mathrm{~s}(0.00 \%) \mathrm{p} 1.00(99.63 \%) \mathrm{d} 0.00(0.37 \%)$

(27.90\%) $0.5282 * \mathrm{~V} 2 \mathrm{~s}(0.00 \%) \mathrm{p} 1.00(11.22 \%) \mathrm{d} 7.82(87.78 \%)$ f $0.09(0.99 \%)$

17. (0.99555) BD ( 1) V 2- F 3

(11.19\%) $0.3345^{*} \mathrm{~V} 2 \mathrm{~s}(34.38 \%)$ p 0.01( 0.24\%)d 1.89( 65.09\%) f $0.01(0.29 \%)$

( 88.81\%) $0.9424 *$ F 3 s( 20.26\%)p 3.93( 79.58\%)d 0.01( 0.16\%)

18. (0.96813) BD (2) V 2- F 3

( 6.24\%) $0.2498 * \mathrm{~V} 2 \mathrm{~s}(0.00 \%) \mathrm{p} 1.00(37.52 \%) \mathrm{d} 1.57(59.01 \%)$ f $0.09(3.47 \%)$

(93.76\%) $0.9683 *$ F 3 s( $0.00 \%) p 1.00(99.87 \%) d 0.00(0.13 \%)$

19. (0.99555) BD ( 1) V 2- F 4

(11.19\%) $0.3345^{*} \mathrm{~V} 2 \mathrm{~s}(34.38 \%) \mathrm{p} 0.01(0.24 \%) \mathrm{d} 1.89(65.09 \%)$ f $0.01(0.29 \%)$

( 88.81\%) $0.9424 *$ F 4 s( 20.26\%)p 3.93( 79.58\%)d 0.01( 0.16\%) 20. (0.96813) BD (2) V 2- F 4

( 6.24\%) $0.2498 * \mathrm{~V} 2 \mathrm{~s}(0.00 \%) \mathrm{p} 1.00(37.52 \%) \mathrm{d} 1.57(59.01 \%)$ f $0.09(3.47 \%)$

(93.76\%) $0.9683 *$ F 4 s( $0.00 \%)$ p 1.00( 99.87\%)d 0.00( $0.13 \%)$

\title{
Beta
}

8. (0.98794) LP ( 1) O $1 \quad$ s( 87.57\%)p 0.14( 12.43\%)d 0.00( $0.00 \%)$ 9. (0.81948) LP (2) O $1 \quad$ s( $0.00 \%)$ p $1.00(99.72 \%) d 0.00(0.28 \%)$

10. (0.99587) LP ( 1) F $3 \quad$ s( $79.90 \%)$ p $0.25(20.09 \%)$ d $0.00(0.00 \%)$ 11. (0.95598) LP (2) F $3 \quad$ s( $0.16 \%)$ p99.99( $99.74 \%)$ d $0.65(0.10 \%)$ 12. (0.99587) LP ( 1) F $4 \quad$ s( $79.90 \%)$ p $0.25(20.09 \%) d 0.00(0.00 \%)$ 13. (0.95598) LP ( 2) F 4 s( $0.16 \%) p 99.99(99.74 \%) d 0.65(0.10 \%)$ 14. (0.99517) BD ( 1$) \mathrm{O} 1-\mathrm{V} 2$

( 73.35\%) $0.8564 * \mathrm{O} 1 \mathrm{~s}(12.56 \%) \mathrm{p} 6.94(87.16 \%) \mathrm{d} 0.02(0.28 \%)$ ( $26.65 \%) \quad 0.5162 * \mathrm{~V} 2 \mathrm{~s}(4.45 \%) \mathrm{p} 0.01(0.05 \%) \mathrm{d} 21.39(95.12 \%)$ f 0.09 ( $0.39 \%)$

15. (0.97530) BD (2) O 1- V 2

( 76.77\%) $0.8762 *$ O $1 \mathrm{~s}(0.00 \%)$ p $1.00(99.68 \%) \mathrm{d} 0.00(0.32 \%)$

(23.23\%) $0.4819 * \mathrm{~V} 2 \mathrm{~s}(0.00 \%) \mathrm{p} 1.00(11.05 \%) \mathrm{d} 7.96(87.98 \%)$ f $0.09(0.97 \%)$

16. (0.99793) BD ( 1) V 2- F 3

\author{
$3.09(0.97 \%)$
}

(11.98\%) $0.3461 * \mathrm{~V} 2 \mathrm{~s}(10.18 \%) \mathrm{p} 0.02(0.20 \%) \mathrm{d} 8.78(89.38 \%)$ f $0.02(0.25 \%)$

( 88.02\%) $0.9382 *$ F 3 s( 19.93\%)p 4.01( 79.90\%)d 0.01( 0.17\%) 17. $(0.97125) \mathrm{BD}(2) \mathrm{V} 2-\mathrm{F} 3$

( 5.38\%) $0.2320 *$ V 2 s( $0.00 \%)$ p $1.00(37.07 \%) d 1.61(59.59 \%)$ f $0.09(3.34 \%)$ 
(94.62\%) $0.9727 *$ F 3 s( $0.00 \%) p 1.00(99.87 \%) d 0.00(0.13 \%)$

18. (0.99793) BD ( 1) V 2- F 4

(11.98\%) $0.3461 * \mathrm{~V} 2 \mathrm{~s}(10.18 \%) \mathrm{p} 0.02(0.20 \%) \mathrm{d} 8.78(89.38 \%)$ f $0.02(0.25 \%)$

( 88.02\%) $0.9382 *$ F 4 s( 19.93\%)p 4.01( 79.90\%)d 0.01( 0.17\%) 19. (0.97125) BD ( 2) V 2- F 4

( 5.38\%) $0.2320 * \mathrm{~V} 2 \mathrm{~s}(0.00 \%) \mathrm{p} 1.00(37.07 \%) \mathrm{d} 1.61(59.59 \%)$ f $0.09(3.34 \%)$

(94.62\%) $0.9727 *$ F 4 s( $0.00 \%) p 1.00(99.87 \%) d 0.00(0.13 \%)$

\section{${ }^{3} \mathrm{OCrF}_{2}$ \\ Alpha}

8. (0.98847) LP ( 1) O $1 \quad$ s( 90.26\%)p 0.11( 9.74\%)d 0.00( $0.00 \%)$

9. (0.75214) LP ( 2) O 1 s( $0.00 \%)$ p 1.00( $99.67 \%)$ d $0.00(0.33 \%)$

10. (0.99776) LP ( 1)Cr 2 s( $0.00 \%)$ p $0.00(0.00 \%) d 1.00(100.00 \%)$ f $0.00(0.00 \%)$

11. (0.99735) LP (2)Cr 2 s( 7.11\%)p 0.00( 0.00\%)d13.05( 92.87\%) f $0.00(0.01 \%)$

12. (0.99654) LP ( 1) F $3 \quad$ s( $85.29 \%)$ p 0.17( $14.71 \%) d 0.00(0.00 \%)$

13. (0.97242) LP (2) F $3 \quad$ s( $0.00 \%)$ p $1.00(99.89 \%)$ d $0.00(0.11 \%)$

14. (0.94221) LP (3) F $3 \quad$ s( $0.27 \%)$ p99.99( $99.63 \%)$ d $0.37(0.10 \%)$

15. (0.99654) LP ( 1) F $4 \quad$ s( $85.29 \%)$ p 0.17( $14.71 \%) d 0.00(0.00 \%)$

16. (0.97242) LP (2) F 4 s( $0.00 \%)$ p $1.00(99.89 \%) d 0.00(0.11 \%)$

17. (0.94221) LP (3) F 4 s( $0.27 \%)$ p99.99(99.63\%)d 0.37( $0.10 \%)$

18. (0.99909) BD ( 1) O 1-Cr 2

(62.58\%) $0.7911 *$ O $1 \mathrm{~s}(0.00 \%)$ p $1.00(99.58 \%) \mathrm{d} 0.00(0.42 \%)$

(37.42\%) $0.6117^{*} \mathrm{Cr} 2 \mathrm{~s}(0.00 \%) \mathrm{p} 1.00(0.33 \%) \mathrm{d} 99.99(99.61 \%)$ f $0.20(0.07 \%)$

19. (0.97913) BD (2) O 1-Cr 2

(69.74\%) $0.8351 *$ O $1 \mathrm{~s}(9.83 \%)$ p 9.15( 89.88\%)d 0.03( 0.30\%)

(30.26\%) $0.5501{ }^{*} \mathrm{Cr} 2 \mathrm{~s}(23.05 \%) \mathrm{p} 0.00(0.06 \%) \mathrm{d} 3.32(76.55 \%)$ f $0.01(0.35 \%)$

20. (0.99255) BD ( 1)Cr 2- F 3

( $14.15 \%) \quad 0.3761{ }^{*} \mathrm{Cr} 2 \mathrm{~s}(34.46 \%) \mathrm{p} 0.01(0.26 \%) \mathrm{d} 1.89(65.07 \%)$ f $0.01(0.21 \%)$

( 85.85\%) $0.9266 * \mathrm{~F} 3 \mathrm{~s}(14.42 \%)$ p 5.92( 85.41\%)d 0.01( 0.18\%) 21. (0.99255) BD ( 1)Cr 2- F 4

( $14.15 \%) \quad 0.3761 * \mathrm{Cr} 2 \mathrm{~s}(34.46 \%) \mathrm{p} 0.01(0.26 \%) \mathrm{d} 1.89(65.07 \%)$ f $0.01(0.21 \%)$

( 85.85\%) $0.9266 *$ F $4 \mathrm{~s}(14.42 \%)$ p 5.92( 85.41\%)d 0.01( 0.18\%)

\section{Beta}

8. (0.98904) LP ( 1) O 1

9. (0.82785) LP ( 2) O 1

10. (0.99672) LP ( 1) F 3 s( 87.05\%)p 0.15( 12.94\%)d 0.00( $0.00 \%)$ s( $0.00 \%)$ p 1.00( 99.71\%)d 0.00( $0.29 \%)$ s( 82.28\%)p 0.22( $17.72 \%) d 0.00(0.00 \%)$ 
11. (0.94848) LP ( 2) F 3

s( $0.35 \%)$ p99.99( 99.55\%)d $0.30(0.10 \%)$

12. (0.99672) LP ( 1) F $4 \quad$ s( $82.28 \%)$ p $0.22(17.72 \%) d 0.00(0.00 \%)$

13. (0.94848) LP (2) F $4 \quad$ s( $0.35 \%) p 99.99(99.55 \%) d ~ 0.30(0.10 \%)$

14. (0.99617) BD ( 1) O 1-Cr 2

(73.81\%) $0.8591 * \mathrm{O} 1 \mathrm{~s}(13.03 \%) \mathrm{p} 6.65(86.67 \%) \mathrm{d} 0.02(0.30 \%)$

( $26.19 \%) \quad 0.5118 * \mathrm{Cr} 2 \mathrm{~s}(5.03 \%) \mathrm{p} 0.01(0.05 \%) \mathrm{d} 18.82(94.57 \%)$ f $0.07(0.35 \%)$

15. (0.96477) BD (2) O 1-Cr 2

( 79.34\%) $0.8907 *$ O $1 \mathrm{~s}(0.00 \%)$ p $1.00(99.67 \%) \mathrm{d} 0.00(0.33 \%)$

(20.66\%) $0.4545 * \mathrm{Cr} 2 \mathrm{~s}(0.00 \%) \mathrm{p} 1.00(16.92 \%) \mathrm{d} 4.87(82.45 \%)$ f $0.04(0.63 \%)$

16. (0.99829) BD ( 1)Cr 2- F 3

(13.20\%) $0.3633 * \mathrm{Cr} 2 \mathrm{~s}(12.51 \%) \mathrm{p} 0.02(0.22 \%) \mathrm{d} 6.96(87.08 \%)$ f $0.02(0.20 \%)$

( 86.80\%) $0.9317 *$ F 3 s( 17.35\%)p 4.75( 82.47\%)d 0.01( 0.18\%)

17. (0.97440) BD (2)Cr 2- F 3

( $5.40 \%) \quad 0.2323 * \mathrm{Cr} 2 \mathrm{~s}(0.00 \%) \mathrm{p} 1.00(36.45 \%) \mathrm{d} 1.71(62.15 \%)$ f $0.04(1.40 \%)$

(94.60\%) $0.9726 *$ F 3 s( $0.00 \%)$ p $1.00(99.87 \%)$ d $0.00(0.13 \%)$

18. (0.99829) BD ( 1)Cr 2- F 4

(13.20\%) $0.3633^{*} \mathrm{Cr} 2 \mathrm{~s}(12.51 \%) \mathrm{p} 0.02(0.22 \%) \mathrm{d} 6.96(87.08 \%)$ f $0.02(0.20 \%)$

( 86.80\%) $0.9317 * \mathrm{~F} 4 \mathrm{~s}(17.35 \%)$ p $4.75(82.47 \%) \mathrm{d}$ 0.01( 0.18\%) 19. (0.97440) BD (2)Cr 2- F 4

( 5.40\%) $0.2323 * \mathrm{Cr} 2 \mathrm{~s}(0.00 \%) \mathrm{p} 1.00(36.45 \%) \mathrm{d} 1.71(62.15 \%)$ f $0.04(1.40 \%)$

(94.60\%) $0.9726 *$ F 4 s( $0.00 \%)$ p 1.00 ( 99.87\%)d 0.00( 0.13\%)

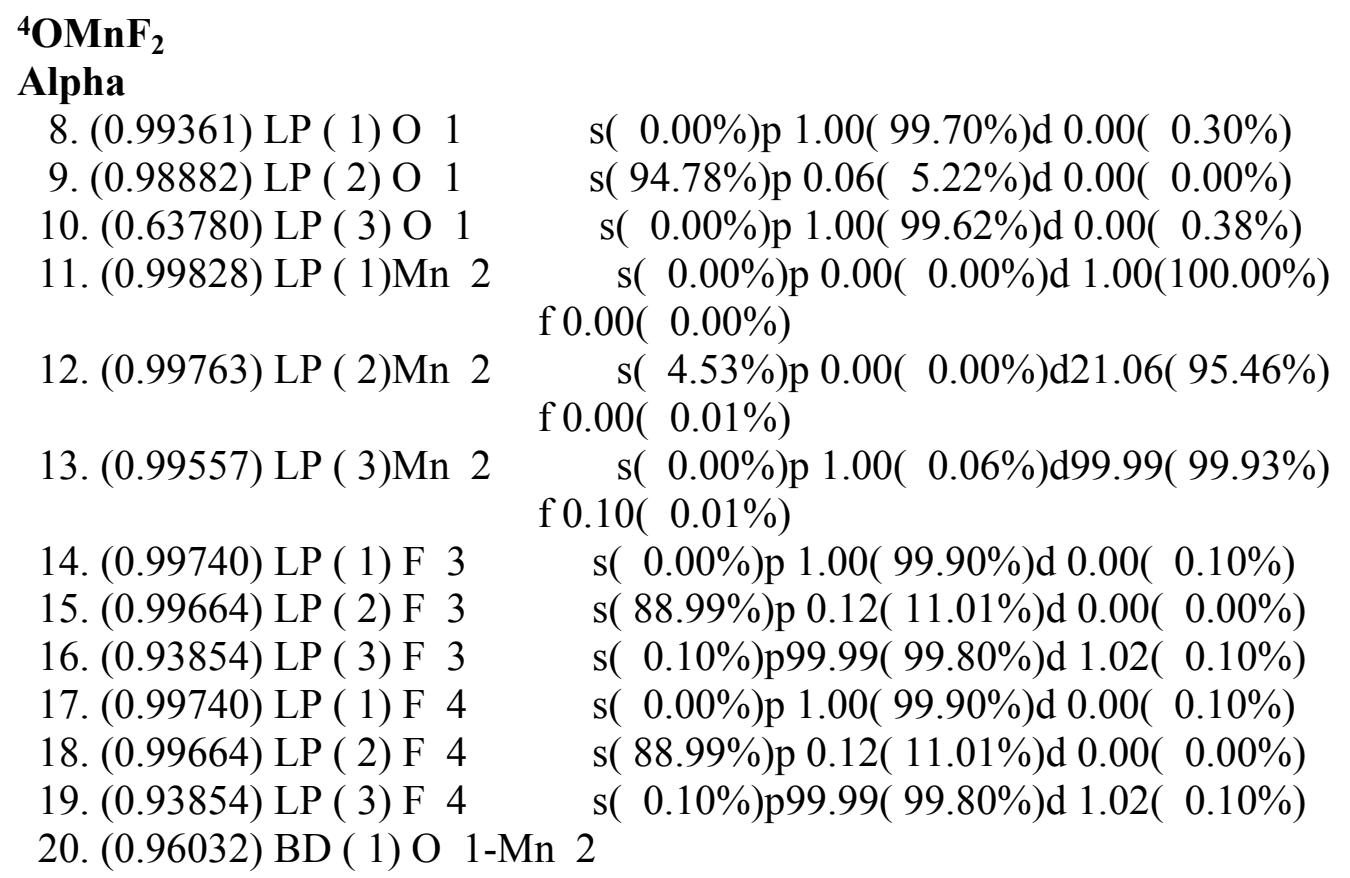


(62.19\%) $0.7886^{*} \mathrm{O} 1 \mathrm{~s}(5.29 \%) \mathrm{p} 17.84(94.28 \%) \mathrm{d} 0.08(0.43 \%)$

( $37.81 \%) \quad 0.6149 * \operatorname{Mn} 2 \mathrm{~s}(24.03 \%)$ p $0.01(0.18 \%) d 3.14(75.54 \%)$ f $0.01(0.24 \%)$

21. (0.98984) BD ( 1)Mn 2- F 3

( $16.35 \%) \quad 0.4044 * \operatorname{Mn} 2 \mathrm{~s}(35.23 \%)$ p $0.01(0.28 \%) d 1.83(64.32 \%)$ f $0.00(0.17 \%)$

( 83.65\%) $0.9146 *$ F $3 \mathrm{~s}(10.88 \%)$ p 8.17( 88.91\%)d 0.02( 0.21\%)

22. (0.98984) BD (1)Mn 2- F 4

( $16.35 \%) \quad 0.4044 * \mathrm{Mn} 2 \mathrm{~s}(35.23 \%)$ p 0.01( $0.28 \%) \mathrm{d} 1.83(64.32 \%)$ f $0.00(0.17 \%)$

(83.65\%) $0.9146 *$ F 4 s( 10.88\%)p 8.17( 88.91\%)d 0.02( 0.21\%)

\section{Beta}

8. (0.99065) LP ( 1) O $1 \quad$ s( 89.73\%)p 0.11( $10.27 \%) d 0.00(0.00 \%)$

9. (0.79942) LP ( 2) O $1 \quad s(0.00 \%)$ p $1.00(99.67 \%) d 0.00(0.33 \%)$

10. (0.99704) LP ( 1) F $3 \quad$ s( 85.06\%)p 0.18( 14.94\%)d 0.00( $0.00 \%)$

11. (0.94551) LP ( 2) F $3 \quad$ s( 0.14\%)p99.99(99.75\%)d 0.75( $0.11 \%)$

12. (0.99704) LP ( 1) F $4 \quad s(85.06 \%)$ p 0.18( $14.94 \%) d 0.00(0.00 \%)$

13. (0.94551) LP ( 2) F $4 \quad s(0.14 \%)$ p99.99(99.75\%)d $0.75(0.11 \%)$

14. (0.99320) BD ( 1) O 1-Mn 2

(72.05\%) $\quad 0.8488 *$ O $1 \mathrm{~s}(10.28 \%)$ p 8.69( 89.32\%)d 0.04( $0.39 \%)$

( $27.95 \%) \quad 0.5287 * \mathrm{Mn} 2 \mathrm{~s}(7.27 \%) \mathrm{p} 0.01(0.09 \%) \mathrm{d} 12.71(92.39 \%)$ f $0.03(0.25 \%)$

15. (0.97301) BD ( 2) O 1-Mn 2

( 74.10\%) $0.8608 *$ O $1 \mathrm{~s}(0.00 \%) \mathrm{p} 1.00(99.61 \%) \mathrm{d} 0.00(0.39 \%)$

(25.90\%) $\quad 0.5089 * \mathrm{Mn} 2 \mathrm{~s}(0.00 \%) \mathrm{p} 1.00(13.38 \%) \mathrm{d} 6.44(86.23 \%)$ f $0.03(0.39 \%)$

16. (0.99749) BD ( 1)Mn 2- F 3

( $14.38 \%) \quad 0.3792 * \operatorname{Mn} 2$ s( $12.48 \%)$ p 0.02 ( $0.23 \%) d$ d 6.98( 87.11\%) f $0.01(0.18 \%)$

( 85.62\%) $0.9253 *$ F 3 s( 14.77\%)p 5.75( 85.01\%)d 0.01( $0.22 \%)$

17. (0.97221) BD ( 2)Mn 2- F 3

( 5.80\%) $\quad 0.2408 * \mathrm{Mn} 2 \mathrm{~s}(0.00 \%)$ p 1.00( 39.99\%)d 1.46( 58.51\%) f $0.04(1.49 \%)$

(94.20\%) $0.9706 *$ F 3 s( $0.00 \%) p 1.00(99.87 \%) d 0.00(0.13 \%)$

18. (0.99749) BD ( 1)Mn 2- F 4

( $14.38 \%) \quad 0.3792 * \operatorname{Mn} 2$ s( $12.48 \%)$ p $0.02(0.23 \%) d$ 6.98( $87.11 \%)$ f $0.01(0.18 \%)$

( 85.62\%) $0.9253 * \mathrm{~F} 4 \mathrm{~s}(14.77 \%)$ p 5.75( 85.01\%)d 0.01( $0.22 \%)$

19. (0.97221) BD ( 2)Mn 2- F 4

( 5.80\%) $0.2408 * \mathrm{Mn} 2 \mathrm{~s}(0.00 \%) \mathrm{p} 1.00(39.99 \%) \mathrm{d} 1.46(58.51 \%)$ f $0.04(1.49 \%)$

(94.20\%) $0.9706 *$ F 4 s( $0.00 \%)$ p $1.00(99.87 \%)$ d $0.00(0.13 \%)$ 


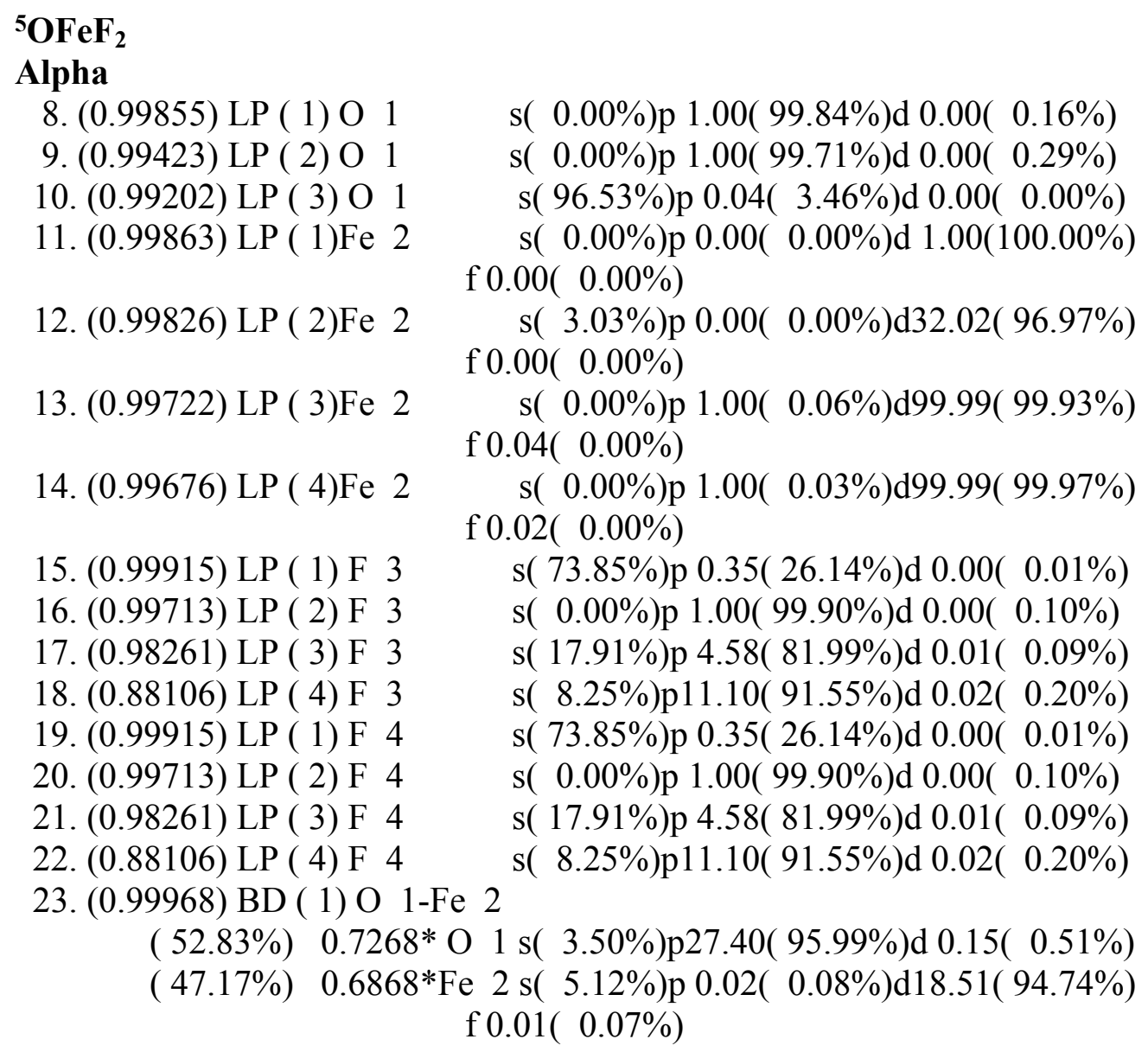

\section{Beta}

8. (0.99160) LP ( 1) O $1 \quad s(93.22 \%)$ p 0.07( 6.77\%)d $0.00(0.01 \%)$

9. (0.99725) LP ( 1) F $3 \quad$ s( 88.11\%)p 0.13(11.89\%)d 0.00( $0.00 \%)$

10. (0.95011) LP ( 2) F $3 \quad$ s( $0.12 \%)$ p99.99(99.78\%)d $0.89(0.10 \%)$

11. (0.99725) LP ( 1) F $4 \quad$ s( 88.11\%)p 0.13( 11.89\%)d 0.00( $0.00 \%)$

12. (0.95011) LP ( 2) F $4 \quad$ s( $0.12 \%)$ p99.99(99.78\%)d 0.89( $0.10 \%)$

13. (0.98711) BD ( 1) O 1-Fe 2

$(67.78 \%) \quad 0.8233 *$ O 1 s( $6.75 \%) p 13.74(92.75 \%) d 0.07(0.50 \%)$

(32.22\%) $\quad 0.5676 * \mathrm{Fe} 2 \mathrm{~s}(10.14 \%) \mathrm{p} 0.01(0.09 \%) \mathrm{d} 8.83(89.59 \%)$ f $0.02(0.17 \%)$

14. (0.97647) BD ( 2) O 1-Fe 2

$(55.89 \%) \quad 0.7476 *$ O 1 s( $0.00 \%)$ p $1.00(99.57 \%) d 0.00(0.43 \%)$

(44.11\%) $0.6642 * \mathrm{Fe} 2 \mathrm{~s}(0.00 \%) \mathrm{p} 1.00(7.66 \%) \mathrm{d} 12.03(92.18 \%)$ f $0.02(0.16 \%)$

15. (0.93890) BD ( 3) O 1-Fe 2

$(71.25 \%) \quad 0.8441 *$ O $1 \mathrm{~s}(0.00 \%) \mathrm{p} 1.00(99.64 \%) \mathrm{d} 0.00(0.36 \%)$

( $28.75 \%) \quad 0.5362 * \mathrm{Fe} 2 \mathrm{~s}(0.00 \%) \mathrm{p} 1.00(21.84 \%) \mathrm{d} 3.54(77.39 \%)$

16. (0.95963) BD ( 1)Fe 2- F 3

$$
\text { f } 0.04(0.77 \%)
$$

( 7.64\%) $0.2763 * \mathrm{Fe} 2 \mathrm{~s}(0.00 \%) \mathrm{p} 1.00(43.31 \%) \mathrm{d} 1.28(55.27 \%)$ 
f $0.03(1.43 \%)$

(92.36\%) $0.9611 * \mathrm{~F} 3 \mathrm{~s}(0.00 \%) \mathrm{p} 1.00(99.86 \%) \mathrm{d} 0.00(0.14 \%)$

17. (0.95023) BD (2)Fe 2- F 3

( $9.82 \%) \quad 0.3133 * \mathrm{Fe} 2 \mathrm{~s}(10.54 \%) \mathrm{p} 3.41$ ( 35.93\%)d $4.90(51.70 \%)$ f $0.17(1.83 \%)$

(90.18\%) $0.9496 * \mathrm{~F} 3 \mathrm{~s}(11.76 \%)$ p 7.48( 88.00\%)d 0.02( $0.25 \%)$

18. (0.95963) BD ( 1)Fe 2- F 4

( 7.64\%) $\quad 0.2763 * \mathrm{Fe} 2 \mathrm{~s}(0.00 \%) \mathrm{p} 1.00(43.31 \%) \mathrm{d} 1.28(55.27 \%)$ f $0.03(1.43 \%)$

(92.36\%) $0.9611 *$ F $4 \mathrm{~s}(0.00 \%) p 1.00(99.86 \%) d 0.00(0.14 \%)$ 19. $(0.95023) \mathrm{BD}(2) \mathrm{Fe} 2-\mathrm{F} 4$

( 9.82\%) $0.3133 *$ Fe 2 s( 10.54\%)p 3.41( 35.93\%)d 4.90( 51.70\%) f $0.17(1.83 \%)$

(90.18\%) $0.9496 *$ F 4 s( $11.76 \%)$ p 7.48( 88.00\%)d 0.02( 0.25\%)

\begin{abstract}
${ }^{4} \mathrm{OCoF}_{2}$ Alpha

8. (0.99880) LP ( 1) O $1 \quad$ s( $0.00 \%)$ p $1.00(99.84 \%) d 0.00(0.16 \%)$

9. (0.99655) LP ( 2) O 1 s( $0.00 \%)$ p 1.00( 99.75\%)d 0.00( $0.25 \%)$

10. (0.99094) LP ( 3) O $1 \quad$ s( 95.91\%)p 0.04( 4.09\%)d 0.00( $0.00 \%)$

11. (0.99868) LP ( 1)Co $2 \quad s(0.00 \%)$ p $0.00(0.00 \%) d 1.00(100.00 \%)$ f $0.00(0.00 \%)$

12. (0.99817) LP (2)Co $2 \quad s(3.16 \%)$ p 0.00( $0.00 \%) d 30.69(96.84 \%)$ f $0.00(0.00 \%)$

13. (0.99766) LP ( 3)Co $2 \quad$ s( $0.00 \%)$ p 1.00( 0.03\%)d99.99( 99.97\%) f $0.06(0.00 \%)$

14. (0.99675) LP (4)Co $2 \quad$ s( $0.00 \%)$ p 1.00( 0.03\%)d99.99( 99.97\%) f $0.02(0.00 \%)$

15. (0.99921) LP ( 1) F $3 \quad$ s( 70.04\%)p 0.43(29.94\%)d 0.00( $0.02 \%)$

16. (0.99773) LP ( 2) F $3 \quad$ s( $0.00 \%)$ p $1.00(99.90 \%) d 0.00(0.10 \%)$

17. (0.98028) LP ( 3) F $3 \quad$ s( 22.35\%)p 3.47( 77.55\%)d 0.00( $0.10 \%)$

18. (0.86337) LP ( 4) F $3 \quad s(7.63 \%) p 12.08(92.15 \%) d 0.03(0.22 \%)$

19. (0.99921) LP ( 1) F $4 \quad$ s( 70.04\%)p 0.43(29.94\%)d 0.00( $0.02 \%)$

20. (0.99773) LP ( 2) F $4 \quad$ s( $0.00 \%)$ p 1.00( 99.90\%)d 0.00( $0.10 \%)$

21. (0.98028) LP ( 3) F $4 \quad s(22.35 \%)$ p 3.47( 77.55\%)d 0.00( $0.10 \%)$

22. (0.86337) LP ( 4) F $4 \quad$ s( 7.63\%)p12.08( 92.15\%)d 0.03( 0.22\%)

23. (0.99973) BD ( 1) O 1-Co 2

(54.20\%) $\quad 0.7362 * \mathrm{O} 1 \mathrm{~s}(4.11 \%) \mathrm{p} 23.21(95.37 \%) \mathrm{d} 0.13(0.52 \%)$

( 45.80\%) $0.6768 * \operatorname{Co} 2 \mathrm{~s}(6.06 \%) \mathrm{p} 0.01(0.05 \%) \mathrm{d} 15.49(93.82 \%)$ f $0.01(0.07 \%)$
\end{abstract}

\title{
Beta
}

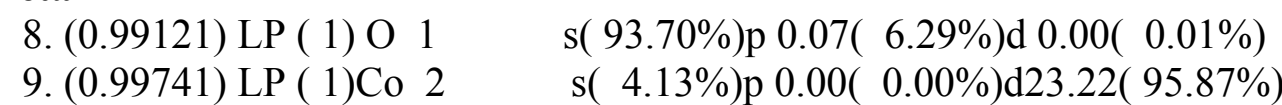




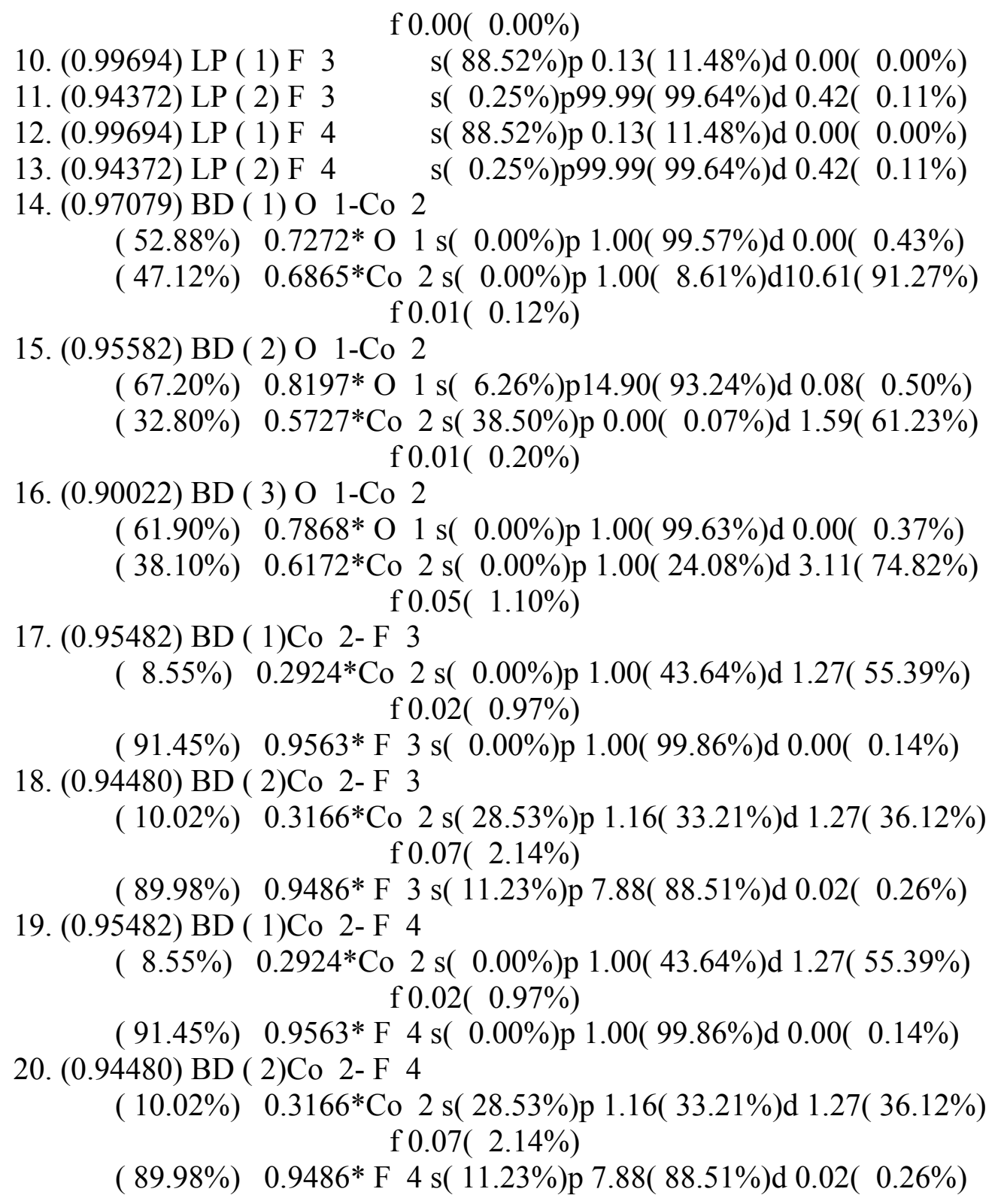


14. (0.99718) LP ( 4)Ni 2

15. (0.99626) LP ( 5)Ni 2

16. (0.99954) LP ( 1) F 3

17. (0.99880) LP ( 2) F 3

18. (0.99775) LP ( 3$) \mathrm{F} 3$

19. (0.95521) LP ( 4) F 3

20. (0.99954) LP ( 1) F 4

21. (0.99880) LP ( 2) F 4

22. (0.99775) LP ( 3) F 4

23. (0.95521) LP ( 4) F 4

24. (0.99940) BD ( 1) O 1-Ni 2

(93.93\%) $0.9692 *$ O $1 \mathrm{~s}(22.77 \%)$ p 3.38( $76.97 \%) d 0.01(0.26 \%)$

( 6.07\%) $0.2463 * \mathrm{Ni} 2 \mathrm{~s}(94.16 \%) \mathrm{p} 0.03(3.17 \%) \mathrm{d} 0.02$ ( 2.35\%)

f $0.00(0.32 \%)$

\section{Beta}

8. (0.99146) LP ( 1) O 1

9. (0.52498) LP ( 2) O 1

10. (0.99745) LP ( 1)Ni 2

11. (0.99724) LP ( 1) F 3

12. (0.93433) LP ( 2) F 3

13. (0.99724) LP ( 1) F 4

14. (0.93433) LP ( 2) F 4

15. (0.95979) BD ( 1) O 1-Ni 2

(62.60\%) $0.7912 *$ O $1 \mathrm{~s}(3.20 \%) \mathrm{p} 30.05(96.22 \%) \mathrm{d} 0.18(0.57 \%)$

(37.40\%) $0.6116^{*} \mathrm{Ni} 2 \mathrm{~s}(35.37 \%) \mathrm{p} 0.00(0.07 \%) \mathrm{d} 1.82(64.46 \%)$ f $0.00(0.10 \%)$

16. (0.92636) BD (2) O 1-Ni 2

s( $96.75 \%)$ p $0.03(3.24 \%) d 0.00(0.01 \%)$

s( $0.00 \%)$ p 1.00 ( 99.70\%)d $0.00(0.30 \%)$

s( $2.62 \%) p$ p $0.00(0.00 \%) \mathrm{d} 37.11(97.37 \%)$ f $0.00(0.00 \%)$

s( $92.41 \%)$ p $0.08(7.59 \%) d 0.00(0.00 \%)$

s( $0.65 \%)$ p99.99( $99.24 \%)$ d $0.16(0.10 \%)$ s( $92.41 \%)$ p $0.08(7.59 \%)$ d $0.00(0.00 \%)$

s( $0.65 \%) p 99.99(99.24 \%) d 0.16(0.10 \%)$

(31.74\%) $0.5634 * \mathrm{O} 1 \mathrm{~s}(0.00 \%) \mathrm{p} 1.00(99.62 \%) \mathrm{d} 0.00(0.38 \%)$

(68.26\%) $0.8262 * \mathrm{Ni} 2 \mathrm{~s}(0.00 \%)$ p 1.00( 13.37\%)d 6.47( 86.55\%)

17. (0.99125) BD ( 1)Ni 2- F 3

$$
\text { f } 0.01(0.08 \%)
$$

(20.46\%) $\quad 0.4523 * \mathrm{Ni} 2 \mathrm{~s}(30.89 \%)$ p 0.02( $0.55 \%) \mathrm{d} 2.22(68.49 \%)$ f $0.00(0.07 \%)$

( 79.54\%) $0.8918 * \mathrm{~F} 3 \mathrm{~s}(6.94 \%) p 13.37(92.79 \%) \mathrm{d}$ 0.04( $0.27 \%)$ 18. (0.93104) BD ( 2)Ni 2- F 3

(13.77\%) $0.3711 * \mathrm{Ni} 2 \mathrm{~s}(0.00 \%) \mathrm{p} 1.00(42.38 \%) \mathrm{d} 1.35(57.31 \%)$ f $0.01(0.31 \%)$

( 86.23\%) $0.9286 *$ F 3 s( $0.00 \%) p 1.00$ ( 99.86\%)d 0.00 ( 0.14\%)

19. (0.99125) BD ( 1)Ni 2- F 4

(20.46\%) $0.4523 * \mathrm{Ni} 2 \mathrm{~s}(30.89 \%)$ p 0.02( $0.55 \%) \mathrm{d} 2.22(68.49 \%)$ f $0.00(0.07 \%)$

( 79.54\%) $0.8918 *$ F 4 s( 6.94\%)p13.37( 92.79\%)d 0.04( 0.27\%) 
20. (0.93104) BD ( 2)Ni 2- F 4

(13.77\%) $0.3711 * \mathrm{Ni} 2 \mathrm{~s}(0.00 \%) \mathrm{p} 1.00(42.38 \%) \mathrm{d} 1.35(57.31 \%)$

f $0.01(0.31 \%)$

( 86.23\%) $0.9286 *$ F 4 s( $0.00 \%)$ p 1.00 ( 99.86\%)d 0.00 ( 0.14\%)

\begin{abstract}
${ }^{4} \mathrm{OCuF}_{2}$
Alpha

8. (0.99967) LP ( 1) O $1 \quad$ s( 79.54\%)p 0.26( 20.44\%)d 0.00( $0.03 \%)$

9. (0.99785) LP ( 2) O 1 s( $0.00 \%)$ p 1.00( $99.83 \%)$ d $0.00(0.17 \%)$

10. (0.93760) LP (3) O 1 s( 20.49\%)p 3.87( $79.22 \%)$ d $0.01(0.29 \%)$

11. (0.99964) LP ( 1)Cu 2 s( $0.00 \%)$ p 0.00( $0.00 \%)$ d $1.00(100.00 \%)$

12. (0.99944) LP (2)Cu $2 \quad$ s( $0.00 \%)$ p $0.00(0.00 \%) d 1.00(100.00 \%)$ f $0.00(0.00 \%)$

13. (0.99870) LP (3)Cu 2 s( $1.57 \%)$ p 0.00( $0.00 \%) d 62.54(98.42 \%)$ f $0.00(0.00 \%)$

14. (0.99764) LP (4)Cu 2 s( $0.30 \%)$ p 0.14( $0.04 \%) d 99.99(99.66 \%)$ f $0.00(0.00 \%)$

15. (0.99931) LP ( 1) F $3 \quad$ s( $55.35 \%)$ p $0.81(44.62 \%)$ d $0.00(0.03 \%)$

16. (0.99716) LP (2) F $3 \quad$ s( $0.00 \%)$ p $1.00(99.91 \%)$ d $0.00(0.09 \%)$

17. (0.99078) LP (3) F $3 \quad$ s( $41.68 \%)$ p $1.40(58.25 \%)$ d $0.00(0.07 \%)$

18. (0.81682) LP (4) F $3 \quad$ s( $3.02 \%)$ p $32.08(96.75 \%) d 0.08(0.23 \%)$

19. (0.99931) LP ( 1) F $4 \quad$ s( $55.35 \%)$ p $0.81(44.62 \%) d 0.00(0.03 \%)$

20. (0.99716) LP ( 2) F 4 s( $0.00 \%)$ p $1.00(99.91 \%) d 0.00(0.09 \%)$

21. (0.99078) LP (3) F 4 s( $41.68 \%)$ p $1.40(58.25 \%)$ d $0.00(0.07 \%)$

22. (0.81682) LP (4) F $4 \quad$ s( $3.02 \%)$ p32.08( 96.75\%)d 0.08( $0.23 \%)$

23. (0.99907) BD ( 1) O 1-Cu 2

(46.69\%) $0.6833 *$ O $1 \mathrm{~s}(0.00 \%) \mathrm{p} 1.00(99.83 \%) \mathrm{d} 0.00(0.17 \%)$

(53.31\%) $0.7301 * \mathrm{Cu} 2 \mathrm{~s}(0.00 \%) \mathrm{p} 1.00(0.37 \%) \mathrm{d} 99.99(99.63 \%)$ f $0.02(0.01 \%)$
\end{abstract}

\title{
Beta
}

8. (0.99854) LP ( 1) O 1

9. (0.99085) LP ( 2) O 1

10. (0.62519) LP ( 3 ) O 1

11. (0.99965) LP ( 1)Cu 2

12. (0.99862) LP ( 2) Cu 2

13. (0.99794) LP ( 3)Cu 2

14. (0.99965) LP ( 1) F 3

15. (0.96941) LP ( 2) F 3

16. (0.96884) LP ( 3 ) F 3

17. (0.99965) LP ( 1) F 4

s( $0.00 \%)$ p $1.00(99.87 \%)$ d $0.00(0.13 \%)$

s( $98.32 \%)$ p $0.02(1.66 \%) d 0.00(0.01 \%)$

s( $1.63 \%)$ p59.95( 97.85\%)d 0.32( $0.52 \%)$

s( $0.00 \%)$ p $0.00(0.00 \%) d 1.00(100.00 \%)$

f $0.00(0.00 \%)$

s( $1.97 \%)$ p $0.00(0.00 \%) d 49.86(98.03 \%)$

f $0.00(0.00 \%)$

s( $0.00 \%)$ p $1.00(0.06 \%) \mathrm{d} 99.99(99.94 \%)$

f $0.01(0.00 \%)$

s( $78.70 \%)$ p $0.27(21.29 \%) d 0.00(0.01 \%)$

s( $19.72 \%)$ p 4.06( 80.12\%)d 0.01( $0.15 \%)$

s( $0.00 \%)$ p $1.00(99.90 \%) d 0.00(0.10 \%)$

s( $78.70 \%)$ p $0.27(21.29 \%)$ d $0.00(0.01 \%)$ 
18. (0.96941) LP ( 2) F $4 \quad$ s( $19.72 \%)$ p $4.06(80.12 \%)$ d $0.01(0.15 \%)$

19. (0.96884) LP (3) F 4 s( $0.00 \%)$ p $1.00(99.90 \%) d 0.00(0.10 \%)$

20. (0.82325) LP (4) F 4 s( $1.63 \%)$ p60.11(98.20\%)d $0.10(0.17 \%)$

21. (0.99976) BD ( 1) O 1-Cu 2

( $16.15 \%) \quad 0.4019 *$ O $1 \mathrm{~s}(0.00 \%)$ p 1.00( 99.81\%)d 0.00( 0.19\%)

$(83.85 \%) \quad 0.9157^{*} \mathrm{Cu} 2 \mathrm{~s}(0.00 \%) \mathrm{p} 1.00(0.13 \%) \mathrm{d} 99.99(99.86 \%)$ f $0.03(0.00 \%)$

22. (0.95367) BD ( 1)Cu 2- F 3

(18.13\%) $0.4258 * \mathrm{Cu} 2 \mathrm{~s}(46.56 \%) \mathrm{p} 0.05(2.17 \%) \mathrm{d} 1.10(51.20 \%)$ f $0.00(0.07 \%)$

( $81.87 \%) \quad 0.9048 *$ F $3 \mathrm{~s}(1.63 \%) p 60.42(98.21 \%) d$ 0.10( 0.17\%)' 